\title{
Heavy to light Higgs boson decays at NLO in the singlet extension of the Standard Model
}

\author{
F. Bojarski, ${ }^{a}$ G. Chalons, ${ }^{a}$ D. López-Val ${ }^{b}$ and T. Robens ${ }^{c}$ \\ ${ }^{a}$ Laboratoire de Physique Subatomique et de Cosmologie, Université Grenoble-Alpes, CNRS/IN2P3, \\ 53 Rue des Martyrs, F-38026 Grenoble Cedex, France \\ ${ }^{b}$ Center for Cosmology, Particle Physics and Phenomenology CP3, \\ Université catholique de Louvain, \\ Chemin du Cyclotron 2, B-1348 Louvain-la-Neuve, Belgium \\ ${ }^{c}$ IKTP, Technische Universität Dresden, \\ Zellescher Weg 19, D-01069 Dresden, Germany \\ E-mail: francois.bojarski@ens-lyon.fr, chalons@lpsc.in2p3.fr, \\ david.lopezval@uclouvain.be, tania.robens@tu-dresden.de
}

ABSTRACT: We study the decay of a heavy Higgs boson into a light Higgs pair at one loop in the singlet extension of the Standard Model. To this purpose, we construct several renormalization schemes for the extended Higgs sector of the model. We apply these schemes to calculate the heavy-to-light Higgs decay width $\Gamma_{H \rightarrow h h}$ at next-to-leading order electroweak accuracy, and demonstrate that certain prescriptions lead to gauge-dependent results. We comprehensively examine how the NLO predictions depend on the relevant singlet model parameters, with emphasis on the trademark behavior of the quantum effects, and how these change under different renormalization schemes and a variable renormalization scale. Once all present constraints on the model are included, we find mild NLO corrections, typically of few percent, and with small theoretical uncertainties.

KeYwords: Higgs Physics, Beyond Standard Model, Precision QED

ARXIV EPRINT: 1511.08120 


\section{Contents}

1 Introduction $\quad 2$

2 Model setup at the classical level 3

2.1 Classical Lagrangian 3

2.2 Mass spectrum 3

2.3 Input parameters 5

2.4 Gauge-fixing Lagrangian 5

$\begin{array}{lll}2.5 & \text { Interactions } & 7\end{array}$

2.6 Constraints 7

3 Renormalization $\quad 9$

3.1 Setup 9

$\begin{array}{lll}3.2 & \text { Gauge sector } & 13\end{array}$

3.3 Extended Higgs sector 14

$\begin{array}{lll}\text { 3.3.1 Tadpole renormalization } & 14\end{array}$

$\begin{array}{lll}\text { 3.3.2 } & \text { Doublet vev renormalization } & 14\end{array}$

$\begin{array}{ll}\text { 3.3.3 Singlet vev renormalization } & 15\end{array}$

$\begin{array}{ll}\text { 3.3.4 Higgs masses renormalization } & 15\end{array}$

$\begin{array}{ll}\text { 3.3.5 Higgs field renormalization: diagonal parts } & 16\end{array}$

$\begin{array}{ll}\text { 3.3.6 Higgs field renormalization: non-diagonal parts } & 17\end{array}$

4 Heavy-to-light Higgs decay width 22

4.1 Leading-order contribution 22

$\begin{array}{lll}4.2 & \text { Electroweak one-loop corrections } & 23\end{array}$

5 Phenomenology 24

5.1 Computational setup 25

$\begin{array}{ll}5.2 & \text { Scheme choice and gauge invariance } 26\end{array}$

5.3 High-mass region 28

5.4 Low-mass region 33

5.5 Maximal branching ratios 35

$\begin{array}{lll}6 & \text { Summary } & 38\end{array}$

A Analytical details $\quad 39$

$\begin{array}{lll}\text { A.1 Feynman rules } & 39\end{array}$

$\begin{array}{lll}\text { A.2 Trilinear Higgs counterterm } & 40\end{array}$ 


\section{Introduction}

With the discovery of the Higgs boson [1,2], the LHC has finally reached the very frontiers of the Standard Model (SM). Dedicated analyses based on Run I data have so far shown excellent agreement between the observed $125 \mathrm{GeV}$ bosonic resonance and the scalar particle originally postulated by Higgs [3, 4], Englert and Brout [5], and Guralnik, Hagen and Kibble [6]. Notwithstanding, it is an ongoing task to decipher whether such a state corresponds indeed to the SM agent of electroweak (EW) symmetry breaking [7-9], or if alternatively the LHC has unveiled just one Higgs-like state among many others, a composite state, or the overlap of multiple resonances, just to mention few possibilities. Moreover, the state-of-the-art precision in the Higgs coupling extraction lies within the 10-20\% level [10], right at the ballpark of the deviations predicted by popular new physics models. The overall picture strengthens the belief that perhaps the Higgs discovery is in fact our first glimpse at a more fundamental UV complete structure.

The arguably most simple, renormalizable extension of the Higgs sector, is constructed by expanding the SM Lagrangian with one additional spinless real electroweak singlet [11-13]. This adds up one extra Higgs companion to the physical spectrum of the model, providing an excellent framework to guide collider searches for exotic scalars, either via direct production or through off-shell effects. Moreover, the coupling between the doublet and the singlet fields mixes the two neutral Higgs states, leading to rescaled Higgs couplings to the SM particles. A chief prediction for collider phenomenology are the universally suppressed cross sections and partial amplitudes in all Higgs production modes and decay channels.

Another paramount signature of a second Higgs is the possibility of the heavy-to-light Higgs decay mode $H \rightarrow h h[12,14]$. The process is governed by the Higgs self-coupling $\lambda_{H h h}$, and as such it constitutes a direct probe of the scalar potential of the model. If the new Higgs boson is lighter than the SM Higgs, the novel $H \rightarrow h h$ decay distorts all Higgs branching ratios, and thereby its signal strengths, from the SM expectations. Alternatively, if the extra scalar is identified with a heavier Higgs companion and $m_{H}>$ $2 m_{h}, H \rightarrow h h$ can significantly contribute to the heavy Higgs width and lineshape and modify its decay pattern.

In this paper we present a thorough study of the heavy-to-light Higgs decay mode $H \rightarrow h h$ in the singlet extension of the SM, including the complete set of radiative corrections at one loop. Aside from being relevant on its own, we use this process as a physics case to construct and compare different renormalization schemes for the extended Higgs sector of the model. Using the SLOOPS [15-18] general non-linear gauge fixing setup, we illustrate how certain prescriptions still exhibit gauge dependence for physical quantities. Our task carries to completion the steps initiated in our previous publication [19], and sharpen up all theoretical tools necessary to completely characterize the singlet model phenomenology at next-to-leading order (NLO) accuracy.

The remainder of the paper is organized as follows. In section 2 we provide a brief description of the model setup and constraints. In section 3 we discuss in full detail our renormalization setup. We devote section 4 to characterize the general aspects of heavy- 
to-light Higgs decays at leading order and at one loop, while in section 5 we present a detailed phenomenological analysis. We summarize and conclude in section 6. Additional analytical details are provided in the appendix.

\section{Model setup at the classical level}

We construct the singlet extension of the SM by adding one colorless, real scalar field, which transforms as a singlet under the $\mathrm{SU}(2)_{L} \otimes \mathrm{U}(1)_{Y}$ gauge charges [11-13, 19-21]. Such a simple renormalizable extension can be viewed as a simplified model approach to the low-energy Higgs sector of a more fundamental UV completion, for instance the decoupling limit of the Next-to-Minimal Supersymmetric Standard Model (NMSSM) [22], some realizations of GUTs [23], models with additional gauge sectors [24] or hidden valleys [25]. The implications of this model were addressed for the first time in refs. [11-13], and it has been the object of dedicated investigation for the past two decades, displaying a very attractive phenomenology, especially in the context of collider physics, see e.g. [26-58]. It has also been subject to many dedicated searches by the LHC experiments, cf. e.g. [59-64] for recent studies.

\subsection{Classical Lagrangian}

The singlet scalar extension of the SM (denoted as xSM) is defined by the Lagrangian

$$
\mathscr{L}_{\mathrm{xSM}}=\mathscr{L}_{\text {gauge }}+\mathscr{L}_{\text {fermions }}+\mathscr{L}_{\text {Yukawa }}+\mathscr{L}_{\text {scalar }}+\mathscr{L}_{\mathrm{GF}}+\mathscr{L}_{\text {ghost }}
$$

where the gauge boson and fermionic kinetic parts $\mathscr{L}_{\text {gauge,fermions, }}$, as well as the Yukawa interaction $\mathscr{L}_{\text {Yukawa }}$, are given by the respective SM contributions. The gauge-fixing and ghost Lagrangians $\mathscr{L}_{\mathrm{GF} \text {,ghost }}$ will be defined in more detail below. The scalar sector is given by

$$
\mathscr{L}_{\text {scalar }}=\left(\mathcal{D}^{\mu} \Phi\right)^{\dagger} \mathcal{D}_{\mu} \Phi+\partial^{\mu} S \partial_{\mu} S-\mathcal{V}(\Phi, S),
$$

where $\mathcal{D}_{\mu}$ is the covariant derivative and $\mathcal{V}(\Phi, S)$ the scalar potential

$$
\mathcal{V}(\Phi, S)=\mu^{2} \Phi^{\dagger} \Phi+\lambda_{1}\left|\Phi^{\dagger} \Phi\right|^{2}+\mu_{s}^{2} S^{2}+\lambda_{2} S^{4}+\lambda_{3} \Phi^{\dagger} \Phi S^{2} .
$$

The latter corresponds to the most general, $S U_{L}(2) \otimes \mathrm{U}(1)_{Y}$-invariant, renormalizable Lagrangian involving the Higgs doublet $\Phi$ and the singlet $S$ fields, and compatible with an additional discrete $\mathcal{Z}_{2}$ symmetry, that precludes other terms with odd (e.g. cubic) field powers in the potential. By assuming all parameters in eq. (2.3) to be real, we disregard additional sources of $\mathcal{C P}$ violation.

\subsection{Mass spectrum}

The doublet and singlet fields are expanded as

$$
\Phi=\left(\begin{array}{c}
G^{+} \\
\frac{v+\phi_{h}+i G^{0}}{\sqrt{2}}
\end{array}\right) \quad S=\frac{v_{s}+\phi_{s}}{\sqrt{2}}
$$


where $v \equiv \sqrt{2}\langle\Phi\rangle=\left(\sqrt{2} G_{F}\right)^{-1 / 2} \simeq 246 \mathrm{GeV}$ and $v_{s} \equiv \sqrt{2}\langle S\rangle$ stand for their respective vacuum expectation values (vevs). The fields $G^{ \pm}, G^{0}$ denote the charged and neutral Goldstone bosons. Since the singlet transforms trivially under the electroweak gauge group, only the doublet vev $v$ takes part in electroweak symmetry breaking, which proceeds exactly as in the SM case.

The linear terms in the fields $\phi_{h}$ and $\phi_{s}$ from eq. (2.3) lead to the tadpole relations

$$
\begin{aligned}
& T_{\phi_{h}}=\mu^{2} v+v^{3} \lambda_{1}+\frac{1}{2} v v_{s}^{2} \lambda_{3}, \\
& T_{\phi_{s}}=\mu_{s}^{2} v_{s}+v_{s}^{3} \lambda_{2}+\frac{1}{2} v_{s} v^{2} \lambda_{3},
\end{aligned}
$$

by which we can express the minimization condition of the Higgs potential (2.3) as $T_{\phi_{h}, \phi_{s}}=0$.

In turn, the quadratic terms in the Higgs fields may be arranged into a $2 \times 2$ squared mass matrix $\mathcal{M}_{h s}^{2}$. In the gauge basis $\left(\phi_{h}, \phi_{s}\right)$ these take the form

$$
\mathcal{M}_{h s}^{2}=\mathcal{T}_{\phi_{h}, \phi_{s}}+\mathcal{M}_{\phi_{h}, \phi_{s}}^{2},
$$

where the tadpole component $\mathcal{T}_{\phi_{h}, \phi_{s}}$ and the squared mass matrix $\mathcal{M}_{\phi_{h}, \phi_{s}}^{2}$ are defined by

$$
\mathcal{T}_{\phi_{h}, \phi_{s}}=\left(\begin{array}{cc}
\frac{T_{\phi_{h}}}{v} & 0 \\
0 & \frac{T_{\phi_{s}}}{v_{s}}
\end{array}\right), \quad \mathcal{M}_{\phi_{h}, \phi_{s}}^{2}=\left(\begin{array}{ccc}
2 \lambda_{1} v^{2} & \lambda_{3} v v_{s} \\
\lambda_{3} v v_{s} & 2 \lambda_{2} v_{s}^{2}
\end{array}\right) .
$$

Requiring this matrix to be positively defined leads to the stability conditions

$$
\lambda_{1}, \lambda_{2}>0 ; \quad 4 \lambda_{1} \lambda_{2}-\lambda_{3}^{2}>0 .
$$

Once we impose the tadpoles $T_{\phi_{h, s}}$ to vanish, eq. (2.8) can be readily transformed into the (tree-level) Higgs mass basis through the rotation $\mathrm{U}(\alpha) \cdot \mathcal{M}_{\phi_{h}, \phi_{s}}^{2} \cdot U^{-1}(\alpha)=\mathcal{M}_{h H}^{2}=$ $\operatorname{diag}\left(m_{h}^{2}, m_{H}^{2}\right)$, the physical Higgs masses reading

$$
m_{h, H}^{2}=\lambda_{1} v^{2}+\lambda_{2} v_{s}^{2} \mp\left|\lambda_{1} v^{2}-\lambda_{2} v_{s}^{2}\right| \sqrt{1+\tan ^{2}(2 \alpha)} .
$$

We identify the corresponding mass-eigenstates as a light $[h]$ and a heavy $[H] \mathcal{C P}$-even Higgs boson, which are related to the gauge eigenstates through

$$
\left(\begin{array}{c}
h \\
H
\end{array}\right)=U(\alpha)\left(\begin{array}{l}
\phi_{h} \\
\phi_{s}
\end{array}\right)=\left(\begin{array}{cc}
\cos \alpha & -\sin \alpha \\
\sin \alpha & \cos \alpha
\end{array}\right)\left(\begin{array}{l}
\phi_{h} \\
\phi_{s}
\end{array}\right)
$$

where the rotation angle $\alpha$ is defined in the range $-\pi / 2 \leq \alpha \leq \pi / 2$ by

$$
\sin 2 \alpha=\frac{\lambda_{3} v v_{s}}{\sqrt{\left(\lambda_{1} v^{2}-\lambda_{2} v_{s}^{2}\right)^{2}+\left(\lambda_{3} v v_{s}\right)^{2}}}, \quad \cos 2 \alpha=\frac{\lambda_{2} v_{s}^{2}-\lambda_{1} v^{2}}{\sqrt{\left(\lambda_{1} v^{2}-\lambda_{2} v_{s}^{2}\right)^{2}+\left(\lambda_{3} v v_{s}\right)^{2}}} .
$$

Likewise the tadpoles in the gauge basis $\left[T_{\phi_{h}}, T_{\phi_{s}}\right]$ may be rotated into the mass basis $\left[T_{h}, T_{H}\right]$ through $\mathrm{U}(\alpha)$ :

$$
\left(\begin{array}{c}
T_{h} \\
T_{H}
\end{array}\right)=\left(\begin{array}{cc}
\cos \alpha & -\sin \alpha \\
\sin \alpha & \cos \alpha
\end{array}\right)\left(\begin{array}{c}
T_{\phi_{h}} \\
T_{\phi_{s}}
\end{array}\right)
$$


The above equations are of service to recast the quartic couplings in the Higgs potential (2.3) as given by the physical Higgs boson masses $m_{h, H}^{2}$ and the mixing angle $\alpha$,

$$
\begin{aligned}
& \lambda_{1}=\frac{m_{h}^{2}}{2 v^{2}} \cos ^{2} \alpha+\frac{m_{H}^{2}}{2 v^{2}} \sin ^{2} \alpha-\frac{\cos \alpha T_{h}+\sin \alpha T_{H}}{2 v^{3}}+\frac{m_{h H}^{2}}{2 v^{2}} \sin 2 \alpha, \\
& \lambda_{2}=\frac{m_{h}^{2}}{2 v_{s}^{2}} \sin ^{2} \alpha+\frac{m_{H}^{2}}{2 v_{s}^{2}} \cos ^{2} \alpha-\frac{\cos \alpha T_{H}-\sin \alpha T_{h}}{2 v_{s}^{3}}-\frac{m_{h H}^{2}}{2 v_{s}^{2}} \sin 2 \alpha, \\
& \lambda_{3}=\frac{m_{H}^{2}-m_{h}^{2}}{2 v v_{s}} \sin 2 \alpha+\frac{m_{h H}^{2}}{v v_{s}} \cos 2 \alpha .
\end{aligned}
$$

The mixed mass term $m_{h H}^{2}$ denotes the (symmetric) off-diagonal element of the squared mass matrix $\mathcal{M}_{h H}^{2}$, defined in the mass-eigenstate basis. While at tree-level we have $T_{h, H}=$ 0 and $m_{h H}^{2}=0$, keeping these dependencies explicit in eqs. (2.14)-(2.16) will be useful to link the Lagrangian parameter counterterms to the corresponding mass counterterms, as we discuss in section 3. Similarly, it is practical to rephrase the bilinear mass terms $\mu$ and $\mu_{s}$ in eq. (2.3) as

$$
\begin{aligned}
\mu^{2}= & -\frac{1}{2} m_{h}^{2} \cos ^{2} \alpha-\frac{1}{2} m_{H}^{2} \sin ^{2} \alpha-\frac{\left(m_{H}^{2}-m_{h}^{2}\right) v_{s}}{4 v} \sin 2 \alpha \\
& +\frac{3\left(\cos \alpha T_{h}+\sin \alpha T_{H}\right)}{2 v}-\frac{m_{h H}^{2} v_{s}}{2 v} \cos 2 \alpha \\
\mu_{s}^{2}= & -\frac{1}{2} m_{h}^{2} \sin ^{2} \alpha-\frac{1}{2} m_{H}^{2} \cos ^{2} \alpha-\frac{\left(m_{H}^{2}-m_{h}^{2}\right) v}{4 v_{s}} \sin 2 \alpha \\
& -\frac{3\left(\sin \alpha T_{h}-\cos \alpha T_{H}\right)}{2 v_{s}}-\frac{m_{h H}^{2} v}{2 v_{s}} \cos 2 \alpha .
\end{aligned}
$$

\subsection{Input parameters}

The Higgs sector of the model is determined at tree-level by i) the doublet vev, bilinear mass term and quartic self-coupling; ii) their counterparts for the singlet field; and iii) the portal coupling $\lambda_{3}$ between both. The singlet vev is traded as customary via the parameter $\tan \beta \equiv \frac{v_{s}}{v} .{ }^{1}$ With the help of the above relations (2.5)-(2.18), we can conveniently recast them in terms of the following five independent parameters:

$$
m_{h}, m_{H}, \sin \alpha, v, \tan \beta .
$$

Two of them are readily fixed in terms of experimental data: the doublet vev is linked to the Fermi constant through $v^{2}=\left(\sqrt{2} G_{F}\right)^{-1}$, while one of the Higgs masses is given by the $\mathrm{LHC}$ value $125.09 \mathrm{GeV}$ [65]. Overall, we are left with three quantities which define the relevant directions in the new physics parameter space. This is also helpful to identify which physical parameters can be used to fix the renormalization conditions.

\subsection{Gauge-fixing Lagrangian}

Gauge invariance will play an important role when discussing the renormalization of the singlet Higgs sector, in particular in defining a gauge-independent mixed mass counterterm,

\footnotetext{
${ }^{1}$ Note the different conventions for $\tan \beta$ employed in the literature. The definition we adopt herewith is the inverse of that from refs. [19-21].
} 
as we discuss in detail in sections 3.3.6 and 5.2. Such non-linear gauges have proven useful to check the gauge independence of higher order calculations within the SM [66-68], and its supersymmetric extensions [15-18, 69-73]. The gauge-fixing Lagrangian can be written in general as

$$
\mathscr{L}_{\mathrm{GF}}=-\frac{1}{\xi_{W}} F^{+} F^{-}-\frac{1}{2 \xi_{Z}}\left|F^{Z}\right|^{2}-\frac{1}{2 \xi_{A}}\left|F^{A}\right|^{2},
$$

where the functions $F$ depend non-linearly on the Higgs and gauge fields,

$$
\begin{aligned}
F^{ \pm}= & \left(\partial_{\mu} \mp i e \tilde{\alpha} A_{\mu} \mp i g \cos \theta_{W} \tilde{\beta} Z_{\mu}\right) W^{\mu+} \\
& \pm i \xi_{W} \frac{g}{2}\left(v+\tilde{\delta}_{1} h+\tilde{\delta}_{2} H \pm i \tilde{\kappa} G^{0}\right) G^{+}, \\
F^{Z}= & \partial_{\mu} Z^{\mu}+\xi_{Z} \frac{g}{2 \cos \theta_{W}}\left(v+\tilde{\epsilon}_{1} h+\tilde{\epsilon}_{2} H\right) G^{0}, \\
F^{A}= & \partial_{\mu} A^{\mu} .
\end{aligned}
$$

In the above equations $e$ is the electromagnetic coupling constant, $g$ the $\mathrm{SU}(2)_{L}$ coupling constant and $\theta_{W}$ denotes the weak mixing angle. The quantities $\left\{\tilde{\alpha}, \tilde{\beta} \cdots \tilde{\epsilon}_{2}\right\}$ correspond to the generalized gauge-fixing parameters. Setting these parameters to zero leads to the standard linear $R_{\xi}$ gauge fixing, with $\xi_{i}=1$ defining the familiar 't Hooft-Feynman gauge. In our renormalization setup we will take these gauge-fixing terms already as renormalized quantities - in such a way that no additional counterterms $\delta \mathscr{L}_{\mathrm{GF}}$ are introduced for this part of the Lagrangian.

In turn, the ghost Lagrangian $\mathscr{L}_{\text {ghost }}$ is derived by requiring the complete Lagrangian at the quantum level to be invariant under BRST transformations. This means $\delta_{\mathrm{BRST}} \mathscr{L}_{\mathrm{xSM}}=0$ and hence $\delta_{\mathrm{BRST}} \mathscr{L}_{\mathrm{GF}}=-\delta_{\mathrm{BRST}} \mathscr{L}_{\text {ghost }}$. This follows from the fact that by construction the gauge, fermionic, Yukawa and scalar components of $\mathscr{L}_{\mathrm{xSM}}$ are invariant under BRST transformations, as the latter are equivalent to gauge transformations. We consider both $\mathscr{L}_{\mathrm{GF}}$ and $\mathscr{L}_{\text {ghost }}$ to be written in terms of renormalized quantities. The BRST transformations specific to the singlet extension are given by

$$
\begin{aligned}
\delta_{\mathrm{BRST}} G^{0} & =+\frac{g}{2}\left[G^{-} c^{+}+G^{+} c^{-}\right]-\frac{e}{2 c_{W} s_{W}} c^{Z}\left[v+c_{\alpha} h+s_{\alpha} H\right], \\
\delta_{\mathrm{BRST}} G^{ \pm} & =\mp \frac{i g}{2} c^{ \pm}\left[v+c_{\alpha} h+s_{\alpha} H \mp i G^{0}\right] \mp i e\left(c^{A}-\frac{s_{W}^{2}-c_{W}^{2}}{2 s_{W} c_{W}}\right) G^{ \pm}, \\
\delta_{\mathrm{BRST}} h & =c_{\alpha}\left(\frac{i g}{2}\left[G^{-} c^{+}-G^{+} c^{-}\right]+\frac{e}{2 c_{W} s_{W}} c^{Z} G^{0}\right), \\
\delta_{\mathrm{BRST}} H & =s_{\alpha}\left(\frac{i g}{2}\left[G^{-} c^{+}-G^{+} c^{-}\right]+\frac{e}{2 c_{W} s_{W}} c^{Z} G^{0}\right),
\end{aligned}
$$

where $c_{\theta}, s_{\theta}$ are shorthand notations for $\cos \theta, \sin \theta$, while $c^{Z}, c^{ \pm}, c^{A}$ stand for the FaddeevPopov ghost fields associated to the $Z^{0}, W^{ \pm}$and the photon field respectively. Within this particular gauge fixing, we set in practice $\xi_{W, Z, A}=1$. This is convenient since the gauge boson propagators take a very simple form, while we still keep the possibility to check the 
gauge independence of the final result, at the expense of adding new gauge parameterdependent vertices to the model [66]. The gauge independence of a calculation can be examined numerically by varying the non-linear gauge parameters $\tilde{\alpha}, \tilde{\beta} \cdots \tilde{\epsilon}_{2}$. Technically, we perform our implementation of the singlet model with non-linear gauge fixing using LANHEP [74, 75] and Sloops [15-18].

\subsection{Interactions}

The key theoretical structure in the model is the doublet-singlet portal coupling $\mathscr{L}_{\text {xSM }} \supset$ $\lambda_{3}\left(\Phi^{\dagger} \Phi\right) S^{2}$, which is responsible for the Higgs mass eigenstates to be admixtures of the doublet $\phi_{h}$ and the singlet $\phi_{s}$ neutral components. One main consequence of this mixing is the universal depletion of all Higgs boson couplings to the SM particles as

$$
g_{x x y}=g_{x x y}^{\mathrm{SM}}\left(1+\Delta_{x y}\right) \quad \text { with } \quad 1+\Delta_{x y}=\left\{\begin{array}{ll}
\cos \alpha & y=h \\
\sin \alpha & y=H
\end{array} .\right.
$$

This global rescaling is ultimately due to the fact that, owing to electroweak gauge invariance, only the doublet component can couple to the fermions (via ordinary Yukawa interactions) and the gauge bosons (via the gauge covariant derivative). The limits of $\sin \alpha=0$ (resp. $\cos \alpha=0$ ) correspond to the decoupling scenarios for the light (resp. heavy) Higgs bosons, in which all couplings of the additional scalar to SM fields identically vanish. The Higgs self-interactions do not obey such a plain rescaling pattern. Instead, they depend non-trivially on the cross-talk between the singlet and the doublet fields. Analytic expressions for their Feynman rules can be found in the appendix.

\subsection{Constraints}

As discussed above, the singlet model specified by the Lagrangian (2.1) is subject to numerous constraints, which have been explicitly discussed in [19-21]. Although our primary focus in this paper is the structure of the higher order corrections in this model irrespectively of the parameter constraints, we briefly remind the reader which ranges are still feasible from the theoretical and experimental sides - and include all of them in our phenomenological analysis of section 5 . We consider

\section{Theoretical constraints.}

- perturbative unitarity at tree level [76, 77], which is taken into account by diagonalizing the five-dimensional $X \rightarrow Y$ scattering basis, with $X, Y \in\{h h, h H$, $\left.H H W^{+} W^{-}, Z Z\right\} .^{2}$

- perturbativity of the self-couplings in the scalar potential, i.e. $\left|\lambda_{i}\left(\mu_{\text {run }}\right)\right| \leq 4 \pi$, where the couplings are evolved through standard renormalization group equations [79] and evaluated at a reference high-energy scale $\mu_{\text {run }} \sim 4 \times 10^{10} \mathrm{GeV},{ }^{3}$ for the high-mass

\footnotetext{
${ }^{2}$ Electroweak gauge bosons are replaced by Goldstone scalars according to the Goldstone equivalence theorem [78].

${ }^{3}$ This scale has been chosen such that the model still guarantees vacuum stability at a scale slightly larger than the SM breakdown scale for which $\lambda_{1} \leq 0$ in the SM limit $(\sin \alpha=0)$. Requiring validity up to higher scales leads to stronger constraints, cf. the discussion in [20].
} 
and at $\mu_{\text {run }}=v=246 \mathrm{GeV}$ for the low-mass scenario (see $[20,21]$ for a more detailed discussion). The high-mass (resp. low-mass) regions correspond to $m_{H}>2 m_{h}$, with $m_{h}=125.09 \mathrm{GeV}$ (resp. $m_{H}>2 m_{h}$, with $m_{H}=125.09 \mathrm{GeV}$ );

- vacuum stability (cf. eq. (2.9)) up to the same high-energy scale.

\section{Experimental constraints.}

- electroweak parameters $S, T, U$ [80-83] in agreement with the $95 \%$ C.L best-fit values from [84];

- similarly, agreement with the measured value of the $W$-mass at 95\% C.L. (see [19] for more details);

- agreement with collider searches from LEP, Tevatron, and the LHC, as implemented in HiggsBounds [85-87];

- agreement with the Higgs signal strength measurements at 95\% C.L., as implemented in HiggsSignals [88]. In addition, we have applied the constraints from the combined signal strength fit, presented in [89], which lead to $|\sin \alpha| \leq 0.36$ for $m_{H} \geq 152 \mathrm{GeV} .^{4}$

It is interesting to observe the interplay of these different constraints on the overall parameter space. We here only summarize the main features ${ }^{5}$ - a dedicated discussion can be found in [21].

- in the high mass region the leading constraints stem from i) direct searches (for $m_{H} \lesssim$ $300 \mathrm{GeV}) ;{ }^{6}$ ii) the difference between the experimental W-mass measurement and its theoretical prediction [19] (in the intermediate range $M_{H} \in[300 \mathrm{GeV} ; 800 \mathrm{GeV}]$ ); and iii) perturbativity of the self-couplings in the scalar potential (for $m_{H} \geq 800 \mathrm{GeV}$ ). All these features are summarized in figure 1 and table 1;

- in the low mass region where $m_{H} \sim 125 \mathrm{GeV}$, the parameter space is extremely constrained, especially from demanding agreement with the LHC Higgs signal strength measurement and the LEP constraints. In table 2 we summarize these constraints. Note that in this regime the SM limit corresponds to $|\sin \alpha|=1$.

Tables 1 and 2 show the current constraints for the maximal (minimal) allowed values of $\sin \alpha$ and $\tan \beta$, following the analysis presented in [21]. Note that the minimal $\tan \beta$

\footnotetext{
${ }^{4}$ A detailed discussion of the determination of limits from the Higgs signal strength can be found in [21]. For $m_{H} \leq 152 \mathrm{GeV}$, we test a two-scalar hypothesis versus the LHC data, leading to an $m_{H}$-dependence for the respective $\chi^{2}$. Results in [89], however, are derived under an SM assumption. For $m_{H} \geq 152 \mathrm{GeV}$, the $\chi^{2}$ is independent of the second resonance mass and in this range we therefore adopt the improved combined experimental limit.

${ }^{5}$ See also [90].

${ }^{6}$ Note that the most recent experimental searches published in 2015 have not been included. These potentially influence the allowed regions for $m_{H} \lesssim 300 \mathrm{GeV}$. Indeed, preliminary studies show that results from [62] especially modify constraints for $m_{H} \leq 250 \mathrm{GeV}$ [91].
} 


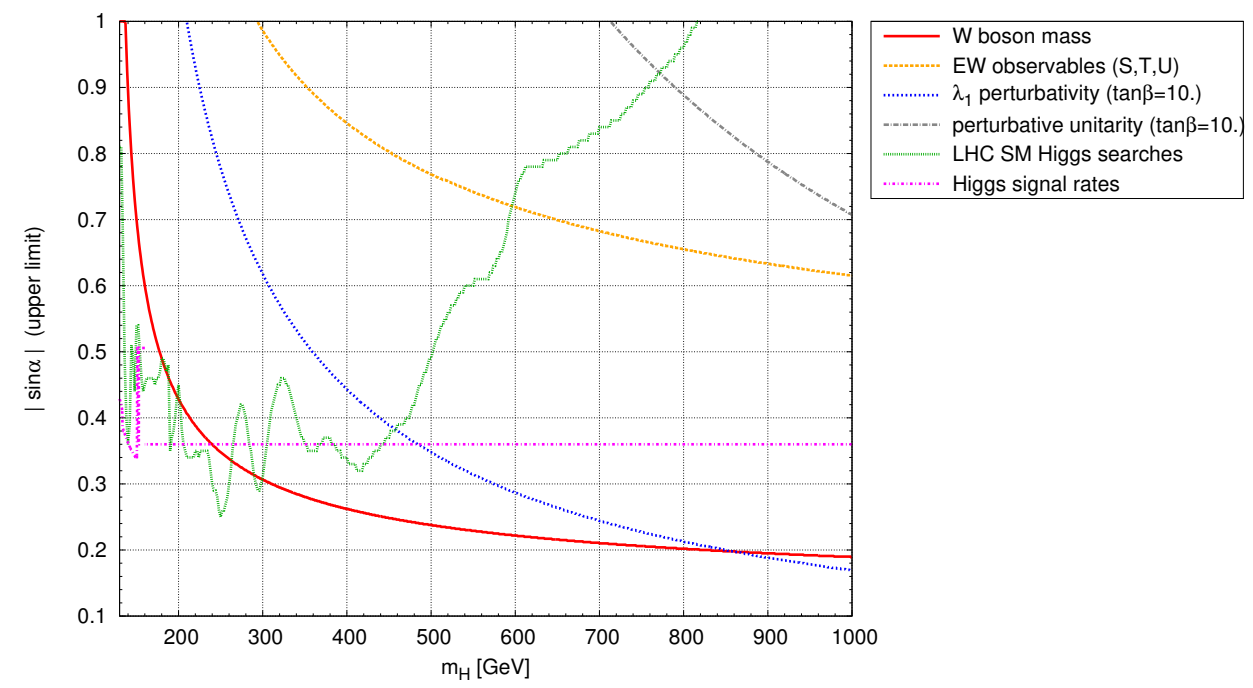

Figure 1. Maximal allowed values for $|\sin \alpha|$ in the high mass region, for a heavy Higgs boson mass in the range $m_{H} \in[130,1000] \mathrm{GeV}$, from the following constraints: i) $W$ boson mass measurement (red, solid) [19]; ii) electroweak precision observables (EWPOs) tested via the oblique parameters $S, T$ and $U$ (orange, dashed); iii) perturbativity, of the RG-evolved coupling $\lambda_{1}$ (blue, dotted), evaluated for an exemplary choice $\tan \beta=10$, iv) perturbative unitarity (grey, dash-dotted), v) direct LHC searches (green, dashed), and vi) Higgs signal strength measurement (magenta, dashdotted). For Higgs masses $m_{H} \in[300 \mathrm{GeV} ; 800 \mathrm{GeV}]$ the $W$ boson mass measurement yields the strongest constraint [19]. The present plot corresponds to an update of figure 8 from [21], where the latest constraints from the combined signal strength [89] have been taken into account.

values shown here were taken at a fixed value of $\sin \alpha$, so results from a generic scan might slightly differ. All the constraints mentioned above have been taken into account when considering viable parameter space regions of the model for our numerical analysis in section 5. Also the results from the combined ATLAS and CMS signal strength fit have been included when applicable. We expect the results from the most recent LHC searches to influence the global picture in the mass region $m_{H} \lesssim 350-400 \mathrm{GeV}$, while for higher values the $W$ boson mass still poses the strongest constraint on the mixing angle.

\section{Renormalization}

\subsection{Setup}

The renormalization program we present here sticks close to the general strategy followed in multidoublet Higgs extensions such as the MSSM [17, 93] and the Two-Higgs-Doublet Model [94]. We generate the required counterterms by introducing multiplicative renormalization constants to the weak coupling constant, fields, tadpoles, masses and vevs. These are then fixed by as many renormalization conditions as independent parameters are present in the model [95]. We adopt on-shell conditions to renormalize the electroweak gauge parameters $[18,96-99]$ and the diagonal terms of the Higgs boson mass matrices. Using an on-shell scheme, as customary in this context, provides an unambiguous interpre- 


\begin{tabular}{|c|c|c|c|}
\hline$m[\mathrm{GeV}]$ & $|\sin \alpha|$ & source upper limit & $(\tan \beta)_{\min }$ \\
\hline 1000 & {$[0.018,0.17]$} & $\lambda_{1}$ perturbativity & 4.34 \\
900 & {$[0.022,0.19]$} & $\lambda_{1}$ perturbativity & 3.85 \\
800 & {$[0.027,0.21]$} & $m_{W}$ at NLO & 3.45 \\
700 & {$[0.031,0.21]$} & $m_{W}$ at NLO & 3.03 \\
600 & {$[0.038,0.23]$} & $m_{W}$ at NLO & 2.56 \\
500 & {$[0.046,0.24]$} & $m_{W}$ at NLO & 2.13 \\
400 & {$[0.055,0.27]$} & $m_{W}$ at NLO & 1.69 \\
300 & {$[0.067,0.31]$} & $m_{W}$ at NLO & 1.28 \\
200 & {$[0.090,0.36]$} & signal rates & 0.85 \\
180 & {$[0.10,0.36]$} & signal rates & 0.77 \\
160 & {$[0.11,0.36]$} & signal rates & 0.68 \\
140 & {$[0.16,0.31]$} & signal rates & 0.60 \\
\hline
\end{tabular}

Table 1. Table II from [21], with adjusted conventions for $\tan \beta$, and updated constraints on the maximally allowed mixing angle from the combined Higgs signal strength fit [89]. It presents allowed ranges for $\sin \alpha$ and $\tan \beta$ in the high mass region for fixed Higgs masses $m$. The allowed interval of $\sin \alpha$ was determined fixing $(\tan \beta)^{-1}=0.15$. The $95 \%$ C.L. limits on $\sin \alpha$ from the Higgs signal rates are derived from one-dimensional fits and taken at $\Delta \chi^{2}=4$. The lower limit on $\sin \alpha$ always stems from vacuum stability, and the upper limit on $\tan \beta$ always from perturbativity of $\lambda_{2}$, evaluated at $\sin \alpha=0.1$. The source of the most stringent upper limit on $\sin \alpha$ is named in the third column. We fixed $m_{h}=125.1 \mathrm{GeV}$ and the stability and perturbativity were tested at the reference scale $\mu_{\text {run }} \sim 4 \times 10^{10} \mathrm{GeV}$.

tation of the bare parameters in the classical Lagrangian in terms of physically measurable quantities. We also recall that field renormalization constants are not needed if we only require the observables derived from S-matrices to be finite, but not each of the Greens' functions individually. They are nonetheless convenient from the technical viewpoint, as they account for loop corrections to the external legs and less Feynman diagrams have to be explicitly included.

We proceed as customary by splitting the bare Lagrangian of the model (2.1) into the renormalized and the counterterm pieces as $\mathscr{L}^{0}\left(\left\{X^{0}\right\}\right) \rightarrow \mathscr{L}(\{X\})+\delta \mathscr{L}(\{\delta X\})$. Accordingly, we rewrite each of the bare parameters $X^{0}$ as a renormalized part $X$ and its counterterm $\delta X$. For the purpose of this work we only need to deal with the scalar and gauge sectors $\mathscr{L}_{\text {scalar,gauge }}^{0}$, as the other sectors do not feature for the remainder of our discussion. We also recall that the gauge-fixing Lagrangian $\mathscr{L}_{\mathrm{GF}}$ does not contribute to $\delta \mathscr{L}$, since we choose to write it already in terms of renormalized fields and parameters $[17,66]$. The physical parameters of the gauge sector are the electromagnetic coupling constant $e$ and the gauge boson masses $m_{W}, m_{Z}$ that we split as [99],

$$
e^{0} \rightarrow e+\delta Z_{e}, \quad\left(m_{W}^{0}\right)^{2} \rightarrow m_{W}^{2}+\delta m_{W}^{2}, \quad\left(m_{Z}^{0}\right)^{2} \rightarrow m_{Z}^{2}+\delta m_{Z}^{2}
$$




\begin{tabular}{|c|c|c|c|c|}
\hline$m_{h}[\mathrm{GeV}]$ & $|\sin \alpha|_{\min , \mathrm{HB}}$ & $|\sin \alpha|_{\min , \mathrm{HS}}$ & $(\tan \beta)_{\min }$ & $(\tan \beta)_{\text {nо } H \rightarrow h h}$ \\
\hline 120 & 0.410 & 0.918 & 0.12 & - \\
110 & 0.819 & 0.932 & 0.11 & - \\
100 & 0.852 & 0.933 & 0.10 & - \\
90 & 0.901 & - & 0.09 & - \\
80 & 0.974 & - & 0.08 & - \\
70 & 0.985 & - & 0.07 & - \\
60 & 0.978 & 0.996 & 0.06 & 4.76 \\
50 & 0.981 & 0.998 & 0.05 & 5.00 \\
40 & 0.984 & 0.998 & 0.04 & 5.56 \\
30 & 0.988 & 0.998 & 0.03 & 6.25 \\
20 & 0.993 & 0.998 & 0.02 & 8.33 \\
10 & 0.997 & 0.998 & 0.01 & 12.5 \\
\hline
\end{tabular}

Table 2. Table III from [21], with adjusted definition for $\tan \beta$ and updated constraints on the minimally allowed mixing angle from the combined Higgs signal strength fit [89]. It presents limits on $\sin \alpha$ and $\tan \beta$ in the low mass scenario for various light Higgs masses $m_{h}$. The limits on $\sin \alpha$ have been determined at $\tan \beta=1$. The lower limit on $\sin \alpha$ stemming from exclusion limits from LEP or LHC Higgs searches is obtained using HiggsBounds [85-87, 92] and given in the second column. If the lower limit on $\sin \alpha$ obtained from the test against the Higgs signal rates using HiggsSignals [88] results in stricter limits, we display them in the third column. The upper limit on $\tan \beta$ in the fourth column stems from perturbative unitarity for the complete decoupling case $(|\sin \alpha|=1)$. In the fifth column we give the $\tan \beta$ value for which $\Gamma_{H \rightarrow h h}=0$ is obtained, given the maximal mixing angle allowed by the Higgs exclusion limits (second column). At this $\tan \beta$ value, the $|\sin \alpha|$ limit obtained from the Higgs signal rates (third column) is abrogated.

Also the bare parameters appearing in $\mathscr{L}_{\text {scalar }}^{0}$ in the gauge-eigenstate basis are decomposed as

$$
\begin{array}{rlrl}
\lambda_{i}^{0} \rightarrow \lambda_{i}+\delta \lambda_{i} & {[i=1 \cdots 3],} & v^{0} \rightarrow v+\delta v, & v_{s}^{0} \rightarrow v_{s}+\delta v_{s}, \\
\mu^{0} \rightarrow \mu+\delta \mu, & \mu_{s}^{0} \rightarrow \mu_{s}+\delta \mu_{s} . &
\end{array}
$$

A similar splitting is introduced for the Higgs tadpoles $T_{\phi}^{0} \rightarrow T_{\phi}+\delta T_{\phi}\left(\left[\phi=\phi_{h}, \phi_{s}\right]\right)$, which feature explicitly for calculations beyond the leading order. Equivalent expressions can be written trading some of the above bare parameters for more physical ones through the relations given by eqs. (2.14)-(2.18).

In our setup we choose not to renormalize the mixing angle $\alpha$. Instead, we promote the relation between the Higgs eigenstates in the gauge $\left(\phi_{h}, \phi_{s}\right)$ and mass basis $(h, H)$ to be valid to all orders,

$$
\left(\begin{array}{c}
h \\
H
\end{array}\right)^{0}=U(\alpha)\left(\begin{array}{c}
\phi_{h} \\
\phi_{s}
\end{array}\right)^{0} \quad \text { and equivalently } \quad\left(\begin{array}{c}
h \\
H
\end{array}\right)=U(\alpha)\left(\begin{array}{c}
\phi_{h} \\
\phi_{s}
\end{array}\right) .
$$

Doing so, the bare and the physical mixing angle coincide and we need no additional mixing angle counterterm. 
In turn, field renormalization constants for the physical Higgs states are introduced by shifting the bare Higgs fields in the mass basis as

$$
\left(\begin{array}{c}
h \\
H
\end{array}\right)^{0} \rightarrow\left(\begin{array}{cc}
1+\frac{1}{2} \delta Z_{h} & \frac{1}{2} \delta Z_{h H} \\
\frac{1}{2} \delta Z_{H h} & 1+\frac{1}{2} \delta Z_{H}
\end{array}\right)\left(\begin{array}{c}
h \\
H
\end{array}\right)+\mathcal{O}\left(\alpha_{\mathrm{ew}}^{2}\right)
$$

Finally, we introduce the (matrix-valued) Higgs mass counterterm via

$$
\mathcal{M}_{\phi}^{2} \rightarrow \mathcal{M}_{\phi}^{2}+\delta \mathcal{M}_{\phi}^{2}
$$

where the generic index $\phi$ applies to the squared mass matrix in both the gauge and the mass basis. Their respective matrix counterterms are linked through

$$
\delta \mathcal{M}_{h H}^{2}=\mathrm{U}(\alpha) \cdot \delta \mathcal{M}_{\phi_{h}, \phi_{s}}^{2} \cdot \mathrm{U}(-\alpha)=\left(\begin{array}{cc}
\delta m_{h}^{2} & \delta m_{h H}^{2} \\
\delta m_{H h}^{2} & \delta m_{H}^{2}
\end{array}\right)
$$

where the mixed mass counterterms are symmetric $\delta m_{H h}^{2}=\delta m_{h H}^{2}$.

Thus, aside from the purely SM inputs, the renormalization of the scalar sector in the singlet model is completely specified by four renormalization constants for the neutral Higgs fields, the respective singlet and doublet vev counterterms, and five additional counterterms linked to the parameters in the Higgs potential (2.3). In the mass eigenstate basis, these can be traded by:

- tadpoles: $\delta T_{h}, \delta T_{H}$

- Higgs masses: $\delta m_{h}^{2}, \delta m_{H}^{2}$
- vev: $\delta v, \delta v_{s}$

- fields: $\delta Z_{h}, \delta Z_{H}, \delta Z_{h H}, \delta Z_{H h}$.
- mixing: $\delta m_{h H}^{2}$

Defining a renormalization scheme is then tantamount to identifying a set of independent conditions by which to link the above quantities to physical inputs. The renormalization conditions by which we fix these counterterms will rely on the one-point and two-point Greens' functions of physical fields. Depending on the scheme we choose for Higgs field renormalization, not all of the above field renormalization constants will be independent from each other.

A complete renormalization scheme fixes all the counterterms which are necessary to absorb the UV-divergent contributions from loop-level amplitudes, such that one obtains UV finite predictions for physical observables. Another important property of a renormalization scheme is gauge independence. More precisely, maintaining gauge independence when defining a scheme allows to write physical predictions as a function of the input parameters in a way that does not vary when the gauge-fixing is changed. Only in this case one can unambiguously relate physical observables to Lagrangian parameters. In this work we examine different strategies to extend the conventional SM renormalization to the singlet model case, and discuss in detail whether these comply with gauge independence. The Stoops non-linear gauge-fixing setup (cf. eq. (2.20)) turns out to be instrumental in this task. 


\subsection{Gauge sector}

We begin by introducing the on-shell definition of the electroweak mixing angle $\sin ^{2} \theta_{W}=$ $1-m_{W}^{2} / m_{Z}^{2}$, along with the shorthand notations $s_{W}^{2} \equiv \sin ^{2} \theta_{W}, c_{W}^{2} \equiv 1-s_{W}^{2}$ [96]. This relation fixes the weak mixing angle counterterm $\left(s_{W}^{2}\right)^{0} \rightarrow s_{W}^{2}+\delta s_{W}^{2}$ as

$$
\frac{\delta s_{W}^{2}}{s_{W}^{2}}=-\frac{c_{W}^{2}}{s_{W}^{2}}\left(\frac{\delta m_{W}^{2}}{m_{W}^{2}}-\frac{\delta m_{Z}^{2}}{m_{Z}^{2}}\right) .
$$

The weak gauge boson masses are renormalized in the standard on-shell scheme [18, 96-99], i.e. by requiring the real part of the transverse renormalized weak gauge boson self-energies to vanish at the respective pole masses. The condition

$$
\operatorname{Re} \hat{\Sigma}_{T}^{V}\left(p^{2}\right)=\operatorname{Re} \Sigma_{T}^{V}\left(p^{2}\right)+\delta Z_{V}\left(p^{2}-m_{V}^{2}\right)-\left.\delta m_{V}^{2}\right|_{p^{2}=m_{V}^{2}}=0 \quad\left[V=W^{ \pm}, Z\right],
$$

where $\delta Z_{V}$ stands for the weak gauge boson field renormalization $V \rightarrow Z_{V}^{1 / 2} V=(1+$ $\left.1 / 2 \delta Z_{V}\right) V+\mathcal{O}\left(\alpha_{\text {ew }}^{2}\right)$, leads to

$$
\delta m_{W}^{2}=-\operatorname{Re} \Sigma_{T}^{W}\left(m_{W}^{2}\right) \quad \text { and } \quad \delta m_{Z}^{2}=-\operatorname{Re} \Sigma_{T}^{Z}\left(m_{Z}^{2}\right) .
$$

All renormalized self-energies are denoted hereafter by a hat. The transverse part of the gauge boson self-energies follows from the vacuum polarization tensor,

$$
\Sigma_{V V^{\prime}}^{\mu \nu}\left(p^{2}\right) \equiv \Sigma_{T}^{V V^{\prime}}\left(p^{2}\right)+p^{\mu} p^{\nu} \Sigma_{L}^{V V^{\prime}}\left(p^{2}\right) .
$$

The explicit form of the weak gauge boson two-point functions in the singlet model can be found in ref. [19].

To renormalize the electromagnetic coupling constant, we require the electric charge to be equal to the full eer vertex in the Thompson limit. With the help of the QED Ward identities, this condition is given in terms of the photon and mixed $Z-\gamma$ two-point functions

$$
\frac{\delta Z_{e}}{e}=\frac{1}{2} \Pi_{\gamma}(0)+\frac{s_{W}}{c_{W}} \frac{\Sigma_{\gamma Z}^{T}(0)}{m_{Z}^{2}}, \quad \text { with } \quad \Pi_{\gamma}=\left.\frac{d^{2}}{\partial p^{2}} \Sigma_{\gamma \gamma}\left(p^{2}\right)\right|_{p^{2}=0} .
$$

To avoid large logarithms from light fermion masses, we rephrase as customary the photon vacuum polarization as

$$
\Pi_{\gamma}(0)=\Delta \alpha_{\text {lep }}+\Delta \alpha_{\text {had }}+\frac{1}{m_{Z}^{2}} \operatorname{Re} \Sigma_{\gamma}^{\text {light f }}\left(m_{Z}^{2}\right),
$$

where the superindex indicates that only the light fermion contributions (all leptons and quarks, except the top) are included in the photon self-energy, while the QED-induced shift to the fine structure constant,

$$
\Delta \alpha=\Delta \alpha_{\text {lep }}+\Delta \alpha_{\text {had }}=-\operatorname{Re} \hat{\Pi}_{\gamma}^{\text {lep }}\left(m_{Z}^{2}\right)-\operatorname{Re} \hat{\Pi}_{\gamma}^{\text {had }}\left(m_{Z}^{2}\right),
$$

is known to very good accuracy $[100,101]$. 
The improved electric charge counterterm in the Thompson limit is thus given by

$$
\frac{\delta Z_{e}}{e}=\left.\frac{1}{2} \frac{d^{2}}{d p^{2}} \Sigma_{\gamma}^{\text {no light f }}\left(p^{2}\right)\right|_{p^{2}=0}+\frac{1}{2} \Delta \alpha+\frac{1}{2 m_{Z}^{2}} \operatorname{Re} \Sigma_{\gamma}^{\operatorname{light~}}\left(m_{Z}^{2}\right)+\frac{s_{W}}{c_{W}} \frac{\Sigma_{\gamma Z}^{T}(0)}{m_{Z}^{2}},
$$

in such a way that the value of the renormalized electric charge at zero momentum transfer $e(0)=\sqrt{4 \pi \alpha_{\mathrm{em}}(0)}$ can be extracted from the measured fine-structure constant in this limit: $\alpha_{\mathrm{em}}(0)=1 / 137.035999074(44)[102]$.

On the other hand, the very precise measurement of the muon lifetime provides a link between the weak gauge boson masses, the fine structure constant and the Fermi constant. This allows for different input choices to fix the electroweak sector. In our numerical analysis we shall use two alternative parametrizations:

- The $\alpha_{\mathrm{em}}$-parametrization, in which we select $\alpha_{\mathrm{em}}(0)$ and $m_{W, Z}$ as input parameters;

- The $G_{F}$-parametrization, in which we instead replace the W-boson mass by the Fermi constant $G_{F}=1.1663787(6) \times 10^{-5} \mathrm{GeV}^{-2}$ [102], the latter being fixed by the muon lifetime via [98, 103-105].

These two parametrizations are related via the conventional parameter $\Delta r[98,103-107]$ as

$$
m_{\mathrm{W}}^{2}\left(1-\frac{m_{\mathrm{W}}^{2}}{m_{Z}^{2}}\right)=\frac{\pi \alpha_{\mathrm{em}}}{\sqrt{2} G_{F}}(1+\Delta r)
$$

where $m_{W, Z}$ and $s_{W}$ are renormalized in the on-shell scheme. For a detailed analysis of $\Delta r$ in the singlet model cf. ref. [19]. Since $\Delta r$ vanishes at leading-order, both parametrizations are trivially linked at tree-level as $\frac{G_{F}}{\sqrt{2}}=\frac{\pi \alpha_{\mathrm{em}}}{2 m_{W}^{2} s_{W}^{2}}$, while they depart from each other at higher perturbative orders. We will explicitly quantify these departures further down in section 5 .

\subsection{Extended Higgs sector}

\subsubsection{Tadpole renormalization}

For the tadpole renormalization we proceed as customary [18, 96-99] and impose

$$
\hat{T}_{h}=T_{h}+\delta T_{h}=0 ; \quad \hat{T}_{H}=T_{H}+\delta T_{H}=0 .
$$

This is equivalent to requiring that $v$ and $v_{s}$ are the physical vacuum expectation values of the doublet and the singlet fields respectively, so that they define the (renormalized) minimum of the Higgs potential. In practice, this implies that no Higgs one-point insertions feature explicitly in our calculation.

\subsubsection{Doublet vev renormalization}

The vev $v$ of the scalar doublet $\Phi$ is fixed as in the SM through its relation to the electroweak on-shell parameters

$$
v=\frac{2 m_{W} s_{W}}{e} \rightarrow \frac{\delta v}{v}=\frac{1}{2} \frac{\delta m_{W}^{2}}{m_{W}^{2}}+\frac{\delta s_{W}}{s_{W}}-\frac{\delta Z_{e}}{e},
$$

where all needed counterterms are defined in (3.10), (3.15) and (3.8). 


\subsubsection{Singlet vev renormalization}

The general renormalization transformation of a generic scalar field vev [108] can be particularized to the singlet vev case as

$$
\phi_{s}+v_{s} \rightarrow Z_{S}^{1 / 2}\left(\phi_{s}+v_{s}+\delta \bar{v}_{s}\right),
$$

where we have introduced for convenience the singlet field renormalization in the gauge basis $S^{0} \rightarrow Z_{S}^{1 / 2} S=\left(1+\delta Z_{S} / 2\right) S+\mathcal{O}\left(\alpha_{\mathrm{em}}^{2}\right)$. The additional counterterm $\delta \bar{v}_{s}$ characterizes to what extent the singlet vev renormalizes differently from the singlet field $\phi_{s}$ itself. In ref. [108] it was shown that, in an $R_{\xi}$ gauge, a divergent part for $\delta \bar{v}_{s}$ is forbidden if the scalar field obeys a rigid invariance (see also ref. [109] and references therein). This is precisely the case in the singlet model, since the singlet field is unlinked from the gauge sector and hence invariant under global gauge transformations. In addition to that, the singlet field renormalization constant $\delta Z_{S}$ is also UV-finite. This can be easily shown by computing $\delta Z_{S}$ in the unbroken phase where $\langle\Phi\rangle=\langle S\rangle=0$. Such a scenario is analogous to a plain $\lambda \phi^{4}$-theory, in which the (singlet) scalar field is coupled to a second scalar (doublet) field only through the gauge-singlet quartic coupling $\mathscr{L} \supset \lambda_{3} \Phi^{\dagger} \Phi S^{2}$. In this case, all oneloop contributions to the singlet two-point function are momentum-independent, implying that $\delta Z_{S}$ does not get a UV pole (cf. e.g. [110]). ${ }^{7}$ We thus conclude that the singlet vev counterterm $\delta v_{s}=\delta \bar{v}_{s}+\delta Z_{S} / 2$ defined by eqs. (3.2) and (3.19) gets at most a finite contribution at this order.

We finally note that, given the condition of vanishing tadpoles (3.17), the singlet vev $v_{s}$ corresponds to the physical minimum of the Higgs potential in the broken phase in the singlet field direction, viz. $\langle S\rangle=v_{s} / \sqrt{2}$, at a given order in perturbation theory. Since $v_{s}$ does not contribute to the electroweak symmetry breaking, it cannot be fixed in terms of SM observables. Instead, we must promote it to an independent input parameter (which should eventually be determined from a future measurement of e.g. the Hhh coupling). So doing, any finite shift $\delta v_{s}$ can be subsumed into the physical definition of $v_{s}$ itself at one-loop. Therefore, in our renormalization setup we can simply fix $\delta v_{s}$ in the $\overline{\mathrm{MS}}$ scheme, $\delta v_{s}^{\overline{\mathrm{MS}}}=0$, so that no singlet vev counterterm features in one-loop calculations.

\subsubsection{Higgs masses renormalization}

The (matrix-valued) Higgs mass counterterm in the gauge basis yields

$$
\delta \mathcal{M}_{\phi_{h}, \phi_{s}}^{2}=\left(\begin{array}{cc}
2\left(v^{2} \delta \lambda_{1}+2 v \lambda_{1} \delta v\right)+\delta T_{\phi_{h}} / v & v_{s}\left(v \delta \lambda_{3}+\lambda_{3} \delta v\right) \\
v_{s}\left(v \delta \lambda_{3}+\lambda_{3} \delta v\right) & 2 v_{s}^{2} \delta \lambda_{2}+\delta T_{\phi_{s}} / v_{s}
\end{array}\right)
$$

where we have already fixed $\delta v_{s}=0$, as justified above. This result can be linked as customary to the mass basis through eq. (3.6).

To renormalize the physical Higgs masses we impose on-shell conditions on the renormalized diagonal Higgs self-energies,

$$
\operatorname{Re} \hat{\Sigma}_{\phi}\left(m_{\phi}^{2}\right)=0 \quad \text { with } \quad \operatorname{Re} \hat{\Sigma}_{\phi}\left(p^{2}\right)=\operatorname{Re} \Sigma_{\phi}\left(p^{2}\right)+\delta Z_{\phi}\left(p^{2}-m_{\phi}^{2}\right)-\delta m_{\phi}^{2}, \quad[\phi=h, H],
$$

\footnotetext{
${ }^{7}$ We have numerically verified that $\delta Z_{S}$ is UV-finite at one loop in all of the different renormalization schemes.
} 
whereby we obtain

$$
\delta m_{h}^{2}=\left.\operatorname{Re} \Sigma_{h}\left(p^{2}\right)\right|_{p^{2}=m_{h}^{2}} \quad \text { and } \quad \delta m_{H}^{2}=\left.\operatorname{Re} \Sigma_{H}\left(p^{2}\right)\right|_{p^{2}=m_{H}^{2}} .
$$

The explicit form of the field renormalization constants $\delta Z_{\phi}$ in different schemes is discussed below in section 3.3.5.

In theories where the gauge eigenstates mix, the renormalization of the non-diagonal or mixing terms must be addressed with care (cf. [17, 18, 93] for an analogue discussion in the context of the squark sector in the MSSM). As we have seen in section 2, a bare angle $\alpha_{0} \equiv \alpha$ rotates the scalar fields from the gauge basis to the mass basis through eq. (2.11). While such diagonal form is valid at leading order, radiative corrections will in general misalign the (tree-level) mass eigenstates. This is reflected in the off-diagonal terms of the loop-corrected propagators,

$$
\Delta_{\text {Higgs }}^{-1}=\left(\begin{array}{cc}
p^{2}-m_{h}^{2}+\hat{\Sigma}_{h}\left(p^{2}\right) & \hat{\Sigma}_{h H}\left(p^{2}\right) \\
\hat{\Sigma}_{H h}\left(p^{2}\right) & p^{2}-m_{H}^{2}+\hat{\Sigma}_{H}\left(p^{2}\right)
\end{array}\right)
$$

traded by the non-diagonal Higgs two-point function

$$
\operatorname{Re} \hat{\Sigma}_{h H}\left(p^{2}\right)=\operatorname{Re} \Sigma_{h H}\left(p^{2}\right)+\frac{1}{2} \delta Z_{h H}\left(p^{2}-m_{h}^{2}\right)+\frac{1}{2} \delta Z_{H h}\left(p^{2}-m_{H}^{2}\right)-\delta m_{h H}^{2} .
$$

One possibility is to absorb these additional quantum effects into the renormalization of the mixing angle. This is equivalent to diagonalizing the loop-corrected mass matrix further through an additional rotation $\mathrm{U}(\delta \alpha)$, where $\delta \alpha$ plays the role of a mixing angle counterterm, such that $\alpha^{0} \rightarrow \alpha+\delta \alpha$. Alternatively, in our approach we take the mixing matrix $\mathrm{U}(\alpha)$ as written in terms of the physical mixing angle and hence valid to all orders. The two alternative approaches are related through

$$
\delta m_{h H}^{2}=\left(m_{H}^{2}-m_{h}^{2}\right) \delta \alpha .
$$

The residual mixing induced by the off-diagonal terms in the mass matrix is instead removed by the non-diagonal field renormalization constants, which we present below.

\subsubsection{Higgs field renormalization: diagonal parts}

Taking the Higgs boson masses $m_{h, H}$ as experimental inputs, we fix the diagonal field renormalization constants via the on-shell conditions

$$
\operatorname{Re} \hat{\Sigma}_{h}^{\prime}\left(m_{h}^{2}\right)=0 \quad \text { and } \quad \operatorname{Re} \hat{\Sigma}_{H}^{\prime}\left(m_{H}^{2}\right)=0
$$

where $\operatorname{Re} \hat{\Sigma}_{\phi}\left(p^{2}\right)$ was defined in eq. (3.21), while the familiar shorthand notation $f^{\prime}\left(p^{2}\right) \equiv$ $d f\left(p^{2}\right) / d p^{2}$ denotes the derivative with the respect to the momentum squared. This leads to

$$
\delta Z_{h}=-\operatorname{Re} \Sigma_{h h}^{\prime}\left(m_{h}^{2}\right) \quad \text { and } \quad \delta Z_{H}=-\operatorname{Re} \Sigma_{H H}^{\prime}\left(m_{H}^{2}\right)
$$

which set the Higgs propagator residues to unity in the limit $p^{2} \rightarrow m_{\phi}^{2}(\phi=h, H)$. 


\subsubsection{Higgs field renormalization: non-diagonal parts}

Fixing the non-diagonal field renormalization is a crucial step in setting up a gaugeinvariant scheme, in which the renormalized one-loop amplitudes are independent of the gauge-fixing parameters, as discussed above. We first construct a set of schemes in analogy to the more familiar approaches in the literature. As we will show, these lead in general to gauge-dependent predictions for physical observables. To circumvent this problem, we introduce an additional (dubbed improved) scheme, which is defined merely in terms of two-point functions and gives numerically stable results throughout the entire parameter space. Similar discussions are addressed e.g. when defining renormalization schemes for the parameter $\tan \beta$ in the MSSM $[17,111]$.

Minimal field. As a first setup to fix the non-diagonal Higgs field renormalization $\delta Z_{h H}$ we resort to a minimal field renormalization. We attach one single renormalization factor per field in the gauge basis,

$$
\Phi \rightarrow Z_{\Phi}^{1 / 2} \Phi=\left(1+\frac{\delta Z_{\Phi}}{2}\right) \Phi+\mathcal{O}\left(\alpha_{\mathrm{ew}}^{2}\right) ; \quad S \rightarrow Z_{S}^{1 / 2} S=\left(1+\frac{\delta Z_{S}}{2}\right) S+\mathcal{O}\left(\alpha_{\mathrm{ew}}^{2}\right),
$$

where we have expanded them to first order.

This procedure is in straight analogy to the conventional renormalization of the Higgs sector in multidoublet extensions such as the MSSM [93] and the Two-Higgs-Doublet Model [94]. Assuming symmetric off-diagonal components, and using the rotation matrix $\mathrm{U}(\alpha)$ in eq. (2.11), we can write the physical Higgs wave function renormalization constants in terms of the gauge basis ones $\delta Z_{\Phi, S}$ as

$$
\begin{aligned}
\delta Z_{h} & =c_{\alpha}^{2} \delta Z_{\Phi}+s_{\alpha}^{2} \delta Z_{S} ; \quad \delta Z_{H}=s_{\alpha}^{2} \delta Z_{\Phi}+c_{\alpha}^{2} \delta Z_{S} \\
\delta Z_{h H} & =s_{\alpha} c_{\alpha}\left(\delta Z_{\Phi}-\delta Z_{S}\right)=\frac{1}{2} t_{2 \alpha}\left[\delta Z_{h}-\delta Z_{H}\right]
\end{aligned}
$$

with the shorthand notation $\left\{s_{\alpha}, c_{\alpha}, t_{\alpha}\right\}=\{\sin \alpha, \cos \alpha, \tan \alpha\}$. The scheme is dubbed minimal as the non-diagonal field renormalization $\delta Z_{h H}$ is not independent. Instead, it is linked to the diagonal parts $\delta Z_{h, H}$, which we have already fixed via on-shell conditions (3.26). Additionally, since at one-loop we have $\delta Z_{S}^{\overline{\mathrm{MS}}}=0$ (cf. section 3.3.3), we can further simplify the relations above to get

$$
\delta Z_{h}=c_{\alpha}^{2} \delta Z_{\Phi} ; \quad \delta Z_{H}=s_{\alpha}^{2} \delta Z_{\Phi} ; \quad \delta Z_{h H}=s_{\alpha} c_{\alpha} \delta Z_{\Phi}=\frac{1}{2} s_{2 \alpha}\left[\delta Z_{h}+\delta Z_{H}\right] .
$$

Finally, for the mixed mass counterterm, which enters explicitly in the $H h h$ vertex counterterm, we demand the off-diagonal renormalized Higgs self-energy to vanish at an arbitrary renormalization scale,

$\left.\operatorname{Re} \hat{\Sigma}_{h H}\left(p^{2}\right)\right|_{p^{2}=\mu_{R}^{2}}=0 ; \quad$ wherefrom $\quad \delta m_{h H}^{2}=\left.\operatorname{Re} \Sigma_{h H}\left(p^{2}\right)\right|_{p^{2}=\mu_{R}^{2}}+\delta Z_{h H}\left(\mu_{R}^{2}-\frac{m_{h}^{2}+m_{H}^{2}}{2}\right)$.

From eq. (3.30) we see that in this scheme all vertices with external Higgs legs receive a finite wave-function renormalization correction, which absorbs the residual loop-induced 
$h-H$ mixing for an external on-shell Higgs state. These finite wave-function factors are given in general by [93]

$$
\begin{aligned}
& \hat{Z}_{h H}=-\frac{\hat{\Sigma}_{h H}\left(m_{h}^{2}\right)}{m_{h}^{2}-m_{H}^{2}+\hat{\Sigma}_{H}\left(m_{h}^{2}\right)}=-\frac{\hat{\Sigma}_{h H}\left(m_{h}^{2}\right)}{m_{h}^{2}-m_{H}^{2}}+\mathcal{O}\left(\alpha_{\mathrm{ew}}^{2}\right) ; \\
& \hat{Z}_{H h}=-\frac{\hat{\Sigma}_{H h}\left(m_{H}^{2}\right)}{m_{H}^{2}-m_{h}^{2}+\hat{\Sigma}_{H}\left(m_{H}^{2}\right)}=-\frac{\hat{\Sigma}_{H h}\left(m_{H}^{2}\right)}{m_{H}^{2}-m_{h}^{2}}+\mathcal{O}\left(\alpha_{\mathrm{ew}}^{2}\right),
\end{aligned}
$$

where $\mathcal{O}\left(\alpha_{\text {ew }}^{2}\right)$ denote the contributions beyond one-loop accuracy. Since the diagonal field renormalization has been fixed via on-shell conditions eq. (3.26), the diagonal finite factors at one loop yield $\hat{Z}_{h, H}=1$ and hence we do not include them explicitly.

On-shell. We define a second prescription in close analogy to squark renormalization $[112-114] .^{8}$ This time we attach one field renormalization constant $\delta Z_{h}, \delta Z_{H}$ per Higgs field directly in the mass-eigenstate basis (3.4), in which case the off-diagonal field renormalization constants $\delta Z_{h H}$ and $\delta Z_{H h}$ are not directly related to the diagonal terms. The diagonal parts $\delta Z_{h, H}$ are again given by the on-shell relations of eq. (3.26). The nondiagonal field renormalization constants are set up by imposing that no loop-induced $H-h$ transitions occur for external Higgs states on their mass shell, i.e.

$$
\operatorname{Re} \hat{\Sigma}_{h H}\left(m_{h}^{2}\right)=0 ; \quad \text { and } \quad \operatorname{Re} \hat{\Sigma}_{h H}\left(m_{H}^{2}\right)=0 .
$$

Using eq. (3.23) leads to

$$
\begin{aligned}
\delta Z_{h H} & =\frac{2}{m_{h}^{2}-m_{H}^{2}}\left[\operatorname{Re} \Sigma_{h H}\left(m_{H}^{2}\right)-\delta m_{h H}^{2}\right], \\
\delta Z_{H h} & =\frac{2}{m_{H}^{2}-m_{h}^{2}}\left[\operatorname{Re} \Sigma_{h H}\left(m_{h}^{2}\right)-\delta m_{h H}^{2}\right] .
\end{aligned}
$$

Therefore, to fully fix the non-diagonal renormalization constants one must provide a proper definition of the mixed mass counterterm $\delta m_{h H}^{2}$. One possibility, as inspired from [112-114], is to impose $\delta Z_{h H}=\delta Z_{H h}$, which fixes $\delta m_{h H}^{2}$ accordingly as

$$
\delta m_{h H}^{2}=\frac{1}{2}\left[\operatorname{Re} \Sigma_{h H}\left(m_{h}^{2}\right)+\operatorname{Re} \Sigma_{h H}\left(m_{H}^{2}\right)\right] \quad \text { and } \quad \delta Z_{h H}=\frac{\operatorname{Re} \Sigma_{h H}\left(m_{H}^{2}\right)-\operatorname{Re} \Sigma_{h H}\left(m_{h}^{2}\right)}{m_{h}^{2}-m_{H}^{2}} .
$$

The above condition removes the loop-induced $H-h$ mixing when either of the two Higgs bosons are on shell, so that the physical states propagate independently and do not oscillate.

The customary on-shell scheme, as well as the minimal field scheme discussed above, show indisputable benefits, e.g. the fact that all counterterms are given in terms of twopoint functions and related to physically measurable quantities. However, both of them lead to renormalized one-loop amplitudes which, albeit UV finite, may still have a leftover dependence on the parameters of the gauge-fixing Lagrangian (2.20). This is a well

\footnotetext{
${ }^{8}$ While this work was being finalized, we learned of the work [115], which presents a study of the quantum corrections to the Higgs couplings to fermions and gauge bosons in a similar singlet model setup. The renormalization scheme for the extended Higgs sector used by these authors is equivalent to the on-shell scheme we discuss here, and which, as we analyse in the following, is not gauge-independent.
} 
known fact for on-shell fermion [116-120] and sfermion mixing in supersymmetric theories $[18,121,122]$. Exploiting the non-linear gauge fixing of eq. (2.20), we explicitly verify this drawback to appear in the singlet model case as well, and illustrate it numerically in section 5.2. In this discussion, it is worthwhile recalling that gauge dependencies may well persist in general in all non-physical building blocks which are involved in the renormalization of any gauge theory (e.g. field renormalization constants). The key test for a given renormalization scheme is thus whether it leads to gauge-independent predictions for physical observables. In the minimal field and the on-shell schemes, renormalized one-loop amplitudes are proven to contain left-over gauge-dependent contributions. These can be traced back to the mixed mass counterterm $\delta m_{h H}^{2}$, which also enters the non-diagonal field renormalization constants $\delta Z_{h H, H h}$. The former is fixed in these schemes through eqs. (3.30) and (3.35) respectively, and ultimately follows from the $h-H$ mixing self-energy. Using the non-linear gauge of (2.20), we find

$$
\begin{aligned}
\Sigma_{h H}\left(p^{2}\right)= & \left.\Sigma_{h H}\left(p^{2}\right)\right|_{\xi_{W}=\xi_{Z}=1, \tilde{\delta}_{i}=0} \\
& +\frac{1}{16 \pi^{2}}\left\{\frac{g^{2}}{2}\left[\tilde{\delta}_{1}\left(m_{H}^{2}-p^{2}\right) s_{\alpha}+\tilde{\delta}_{2}\left(m_{h}^{2}-p^{2}\right) c_{\alpha}\right] B_{0}\left(p^{2}, m_{W}^{2}, m_{W}^{2}\right)\right\} \\
& +\frac{1}{16 \pi^{2}}\left\{\frac{g^{\prime 2}}{4 s_{W}^{2}}\left[\tilde{\epsilon}_{1}\left(m_{H}^{2}-p^{2}\right) s_{\alpha}+\tilde{\epsilon}_{2}\left(m_{h}^{2}-p^{2}\right) c_{\alpha}\right] B_{0}\left(p^{2}, m_{Z}^{2}, m_{Z}^{2}\right)\right\},
\end{aligned}
$$

where $B_{0}\left(p^{2}, m^{2}, m^{2}\right)$ is the two-point Passarino-Veltman scalar integral [123] and the $\tilde{\delta}_{i}$ terms are a short-hand notation for the non-linear gauge parameters in eq. (2.20). The first line of eq. (3.36) is identical to the result of the self-energy computation in the 't HooftFeynman gauge. The second and third lines correspond to the genuine gauge-dependent contributions in the non-linear gauge (we recall that for practical calculations we always set $\left.\xi_{A, W, Z}=1\right)$. The latter enter the mixed mass counterterm definition through eqs. (3.30) or (3.35) and are responsible for the uncancelled dependencies on the gauge-fixing parameters in the renormalized $H \rightarrow h h$ one-loop amplitude, which we pin down numerically in section 5.2.

One first roadway to construct a gauge-independent definition of $\delta m_{h H}^{2}$ alternative to eq. (3.35) would be to exploit the pole structure of a process-specific one-loop amplitude (e.g. a Higgs decay) in the limit $m_{h}^{2} \rightarrow m_{H}^{2}$, as suggested by ref. [18]. Such a limit corresponds though to a vanishing quartic coupling $\lambda_{3}$ (2.16) and hence to a vanishing mixing angle $\alpha$ (2.12). Therefore, in this no-mixing situation, $\delta m_{h H}^{2}$ cannot be defined through the mixed self-energy $\Sigma_{h H}$, because it is identically zero. A second possibility would be to link the problematic mixed mass counterterm to a physical observable directly — viz. using a per se gauge-independent quantity such as a decay rate or scattering cross section $[17,111]$. The price one would pay would be a process-dependent scheme definition, and sometimes one would have to resort to quantities out of current experimental reach. A third option is retaining only the UV-divergent part of such a quantity via an $\overline{\mathrm{MS}}$ prescription, which we examine next. Besides this possibility, we also propose an additional prescription leading to a gauge invariant scheme, which furthermore does not render artificially enhanced contributions in any part of the parameter space. 
Mixed $\overline{\mathbf{M S}}$ /on-shell. In this case we trade $\delta m_{h H}^{2}$ by one of the Higgs self-coupling counterterms $\delta \lambda_{i}$ from eq. (2.3), and fix it using $\overline{\mathrm{MS}}$ conditions. For convenience we choose $\lambda_{2}$ and compute the divergent part of the one-loop correction to the singlet field four-point coupling $\lambda_{2} S^{4}$. So doing we find

$$
\delta \lambda_{2}^{\overline{\mathrm{MS}}}=\frac{-1}{16 \pi^{2}}\left[\lambda_{3}^{2}+9 \lambda_{2}^{2}\right] \Delta
$$

where $\Delta$ stands for the UV divergent part in dimensional regularization

$$
\Delta \equiv 1 / \epsilon-\gamma_{E}+\log (4 \pi)
$$

This result is manifestly gauge independent, as it should, and agrees with the beta function for the singlet quartic coupling $\lambda_{2}$ given in ref. [21]. The corresponding gauge-invariant counterterms for $\lambda_{1,3}$ can now be traded by $\delta m_{h, H}^{2}, \delta v, \delta T_{h}, \delta T_{H}$ and $\delta \lambda_{2}^{\overline{\mathrm{MS}}}$ using the relations from eqs. (2.14)-(2.16),

$$
\begin{aligned}
& \delta \lambda_{1}=\frac{\delta m_{h}^{2}+\delta m_{H}^{2}}{2 v^{2}}+\frac{v s_{\alpha}-v_{s} c_{\alpha}}{2 v^{3} v_{s}} \delta T_{h}-\frac{v_{s} s_{\alpha}+v c_{\alpha}}{2 v^{3} v_{s}} \delta T_{H}-\frac{v_{s}^{2}}{v^{2}} \delta \lambda_{2}^{\overline{\mathrm{MS}}}-\frac{2 \lambda_{1}}{v} \delta v \\
& \delta \lambda_{3}=\frac{c t_{\alpha} \delta m_{H}^{2}-t_{\alpha} \delta m_{h}^{2}}{2 v v_{s}}+\frac{c_{2 \alpha}}{2 v v_{s}^{2}}\left[\frac{\delta T_{h}}{c_{\alpha}}-\frac{\delta T_{H}}{s_{\alpha}}\right]-\frac{2}{t_{2 \alpha}} \frac{v_{s}}{v} \delta \lambda_{2}^{\overline{\mathrm{MS}}}-\frac{\lambda_{3}}{v} \delta v
\end{aligned}
$$

We are thus left with

$$
\begin{aligned}
\delta m_{h H}^{2}= & v^{2} s_{2 \alpha} \delta \lambda_{1}-\delta \lambda_{2}^{\overline{\mathrm{MS}}} v_{s}^{2} s_{2 \alpha}+v v_{s} c_{2 \alpha} \delta \lambda_{3}+\frac{s_{2 \alpha}}{2}\left[\left(\frac{c_{\alpha}}{v}+\frac{s_{\alpha}}{v_{s}}\right) \delta T_{h}+\left(\frac{s_{\alpha}}{v}-\frac{c_{\alpha}}{v_{s}}\right) \delta T_{H}\right] \\
& +\left(2 v s_{2 \alpha} \lambda_{1}+v_{s} c_{2 \alpha} \lambda_{3}\right) \delta v .
\end{aligned}
$$

Finally, we use the on-shell relations (3.33)-(3.34) to obtain the non-diagonal field renormalization constants, which are now fixed in terms of eq. (3.41).

Since all of the renormalization constants within $\delta m_{h H}^{2}$ are either related to physical observables and/or correspond to prefactors of gauge invariant operators (e.g. $\lambda_{1,2,3}$ ) the mixed mass counterterm $\delta m_{h H}^{2}$ is by construction gauge-invariant - and leads in turn to gauge-independent renormalized one-loop amplitudes, as we prove numerically in section 5 . This observation, together with the analytical structure of the mixed self-energy $\Sigma_{h H}\left(p^{2}\right)$ from eq. (3.36), reflects that the renormalization conditions chosen for $\delta m_{h H}^{2}$ (and linked to them, for $\delta Z_{h H, H h}$ ) are the ultimate origin of the uncancelled gauge dependence found in the minimal field and the on-shell schemes.

In spite of leading to gauge-independent results, this mixed $\overline{\mathrm{MS}}$ /on-shell scheme tends to produce overestimated radiative corrections in the phenomenologically interesting regions $\left(s_{\alpha} \rightarrow 0, c_{\alpha} \rightarrow 0\right)$, as manifest from the analytic dependencies of the counterterms (3.39)-(3.40), which are proportional to inverse powers of small trigonometric factors. We therefore refrain from using this scheme explicitly in our phenomenological analysis, and instead propose an improved gauge-independent setup right below. 


\begin{tabular}{|c|c|c|c|}
\hline & $\delta Z_{h, H}$ & $\delta Z_{h H, H h}$ & $\delta m_{h H}^{2}$ \\
\hline Minimal field & $\begin{array}{l}\delta Z_{h}=-\operatorname{Re} \Sigma_{h}^{\prime}\left(m_{h}^{2}\right) \\
\delta Z_{H}=-\operatorname{Re} \Sigma_{H}^{\prime}\left(m_{H}^{2}\right)\end{array}$ & $\begin{aligned} \delta Z_{h H} & =\frac{1}{2} s_{2 \alpha}\left[\delta Z_{h}+\delta Z_{H}\right] \\
\delta Z_{H h} & =\delta Z_{h H}\end{aligned}$ & $\operatorname{Re} \Sigma_{h H}\left(\mu_{R}^{2}\right)+\left[\mu_{R}^{2}-\frac{m_{h}^{2}+m_{H}^{2}}{2}\right]$ \\
\hline OS & $\begin{array}{l}\delta Z_{h}=-\operatorname{Re} \Sigma_{h}^{\prime}\left(m_{h}^{2}\right) \\
\delta Z_{H}=-\operatorname{Re} \Sigma_{H}^{\prime}\left(m_{H}^{2}\right)\end{array}$ & $\begin{aligned} \delta Z_{h H} & =\frac{\operatorname{Re} \Sigma_{h H}\left(m_{H}^{2}\right)-\operatorname{Re} \Sigma_{h H}\left(m_{h}^{2}\right)}{m_{h}^{2}-m_{H}^{2}} \\
\delta Z_{H h} & =\delta Z_{h H}\end{aligned}$ & $\frac{\operatorname{Re} \Sigma_{h H}\left(m_{h}^{2}\right)+\operatorname{Re} \Sigma_{h H}\left(m_{H}^{2}\right)}{2}$ \\
\hline Mixed $\overline{\mathrm{MS}} / \mathrm{OS}$ & $\begin{array}{l}\delta Z_{h}=-\operatorname{Re} \Sigma_{h}^{\prime}\left(m_{h}^{2}\right) \\
\delta Z_{H}=-\operatorname{Re} \Sigma_{H}^{\prime}\left(m_{H}^{2}\right)\end{array}$ & $\begin{aligned} \delta Z_{h H} & =\frac{2}{m_{h}^{2}-m_{H}^{2}}\left[\operatorname{Re} \Sigma_{h H}\left(m_{H}^{2}\right)-\delta m_{h H}^{2}\right] \\
\delta Z_{H h} & =\frac{2}{m_{H}^{2}-m_{h}^{2}}\left[\operatorname{Re} \Sigma_{h H}\left(m_{h}^{2}\right)-\delta m_{h H}^{2}\right]\end{aligned}$ & eq. (3.41) \\
\hline Improved OS & $\begin{array}{l}\delta Z_{h}=-\operatorname{Re} \Sigma_{h}^{\prime}\left(m_{h}^{2}\right) \\
\delta Z_{H}=-\operatorname{Re} \Sigma_{H}^{\prime}\left(m_{H}^{2}\right)\end{array}$ & $\begin{aligned} \delta Z_{h H} & =\frac{2}{m_{h}^{2}-m_{H}^{2}}\left[\operatorname{Re} \Sigma_{h H}\left(m_{H}^{2}\right)-\delta m_{h H}^{2}\right] \\
\delta Z_{H h} & =\frac{2}{m_{H}^{2}-m_{h}^{2}}\left[\operatorname{Re} \Sigma_{h H}\left(m_{h}^{2}\right)-\delta m_{h H}^{2}\right]\end{aligned}$ & $\operatorname{Re} \Sigma_{h H}\left(p_{*}^{2}\right), \quad p_{*}^{2}=\frac{m_{h}^{2}+m_{H}^{2}}{2}$ \\
\hline
\end{tabular}

Table 3. Overview of the scheme-dependent counterterms in the different renormalization setups considered in this paper.

Improved on-shell. A second alternative to sidestep the gauge-dependent $\delta m_{h H}^{2}$ definition in the default on-shell scheme is to isolate the gauge invariant part of the mixed self energy of eq. (3.36). In so doing, we can use it to define the problematic mixed mass counterterm through a gauge-independent improved self-energy [124]. This is actually possible if the mixed scalar self-energy (3.36) is computed in the linear 't Hooft-Feynman gauge and evaluated at the average geometrical mass $p_{*}^{2}=\left(m_{h}^{2}+m_{H}^{2}\right) / 2$. As shown in ref. [122] with the help of the so-called pinch technique, [124-126], the mixed scalar selfenergy (3.36) obtained in this way coincides with the gauge-invariant part of the pinched result. While the results proven in ref. [122] are applied to the squark and Higgs sectors of the MSSM, the proof does not rely on Supersymmetry and hence can be exported to the more general case of a system of two gauge eigenstates which mix in the mass basis. In addition, self-energies computed using the pinch technique are independent of the gaugefixing scheme [124]. With these arguments in mind, we thus retain only the first line in eq. (3.36) and define the mixed mass counterterm through

$$
\delta m_{h H}^{2}=\left.\operatorname{Re} \Sigma_{h H}\left(p_{*}^{2}\right)\right|_{\xi_{W}=\xi_{Z}=1, \tilde{\delta}_{i}=0} \quad \text { with } \quad p_{*}^{2}=\frac{m_{h}^{2}+m_{H}^{2}}{2}
$$

which must be therefore gauge-independent (as we again confirm numerically in section 5.2). Finally, the non-diagonal field renormalization are once more fixed using OS conditions (3.32) and fully determined in terms of $\delta m_{h H}^{2}$.

In table 3 we provide a summarized overview of the different renormalization schemes discussed in this section. Notice that they differ from each other in the renormalization conditions used to fix the non-diagonal Higgs field renormalization $\delta Z_{h H, H h}$ constants and the mixed mass counterterm $\delta m_{h H}^{2}$. 


\section{Heavy-to-light Higgs decay width}

\subsection{Leading-order contribution}

When kinematically accessible, the heavy-to-light Higgs decay mode $H \rightarrow h h$ proceeds at leading order (LO) via the tree-level contact interaction $\lambda_{H h h}$ with partial width $[12,14]$

$$
\Gamma_{H \rightarrow h h}^{\mathrm{LO}}=\frac{\lambda_{H h h}^{2}}{32 \pi m_{H}} \sqrt{1-\frac{4 m_{h}^{2}}{m_{H}^{2}}},
$$

where

$$
\lambda_{H h h}=-\frac{i s_{2 \alpha}}{v}\left[m_{h}^{2}+\frac{m_{H}^{2}}{2}\right]\left(c_{\alpha}+s_{\alpha} t_{\beta}^{-1}\right) .
$$

Notice that, owing to the structure of the scalar self-coupling, the decay width is not symmetric under a sign flip of the mixing angle $s_{\alpha} \rightarrow-s_{\alpha}$.

As such, this decay mode constitutes a genuine new physics contribution to the total heavy Higgs width - aside from the global rescaling of its decay modes into SM particles. The opening of this novel channel is thus capable to alter the Higgs boson lineshape significantly, as well as its decay pattern. More specifically, the branching fractions of the heavy Higgs boson of mass $m_{H}$ to SM fields $\phi$ are modified as

$$
\operatorname{BR}_{H \rightarrow \phi \phi}\left(m_{H}\right)=\frac{s_{\alpha}^{2} \Gamma_{H \rightarrow \phi \phi}^{\mathrm{SM}}\left(m_{H}\right)}{s_{\alpha}^{2} \Gamma_{H_{\text {tot }}}^{\mathrm{SM}}\left(m_{H}\right)+\Gamma_{H \rightarrow h h}\left(m_{H}\right)},
$$

where $\Gamma_{H}^{\mathrm{SM}}\left(m_{H}\right)$ stands for the total width of a SM-like Higgs boson with mass $m_{H}$. For the lighter Higgs boson with mass $m_{h}$, the branching fractions are exactly as for a SM-like Higgs with that mass.

Notice that for $\lambda_{H h h}=0$, all partial decay widths are universally rescaled in terms of the Higgs mixing angle $\alpha$, leading to the same branching ratios that a Higgs boson of that mass would experience in the SM.

Two competing mechanisms determine the overall size of $\Gamma_{H \rightarrow h h}^{\mathrm{LO}}$. On the one hand there is the kinematic factor $\beta_{\text {kin }} / m_{H}^{2}=1 / m_{H}^{2} \sqrt{1-4 m_{h}^{2} / m_{H}^{2}}$, where $\beta_{\text {kin }}$ trades the light Higgs-pair velocity in the heavy Higgs boson rest frame. Its dependence with respect to $m_{H}$ is displayed in the left panel of figure 2, for a fixed light Higgs mass $m_{h}=125.09 \mathrm{GeV}$.

The characteristic $\mathcal{O}\left(m_{H}^{-1}\right)$ phase-space suppression is compensated by the trilinear Higgs coupling strength $\lambda_{H h h}$, which depends quadratically on both the light and the heavy Higgs masses. On the other hand, there are $\cot \beta$-enhanced contributions which can invigorate these Higgs self-interactions for $t_{\beta}<1$, and push the $H \rightarrow h h$ rates even higher. We illustrate these effects in the right panel of figure 2 , in which we show the leading-order heavy-to-light Higgs decay width $\Gamma_{H \rightarrow h h}^{\mathrm{LO}}$ in the $s_{\alpha}-t_{\beta}$ plane for a heavy Higgs boson with mass $m_{H}=300 \mathrm{GeV}$. We can identify three different configurations in which the $H \rightarrow h h$ mode exactly vanishes [21]: i) the light Higgs decoupling limit, $s_{\alpha}=0$; ii) the heavy Higgs decoupling limit, $\left|s_{\alpha}\right|=1$; and iii) the line $t_{\beta}=-t_{\alpha}$. In cases i (resp. ii), all couplings of the heavy (resp. light) Higgs boson eigenstate are identically zero. 

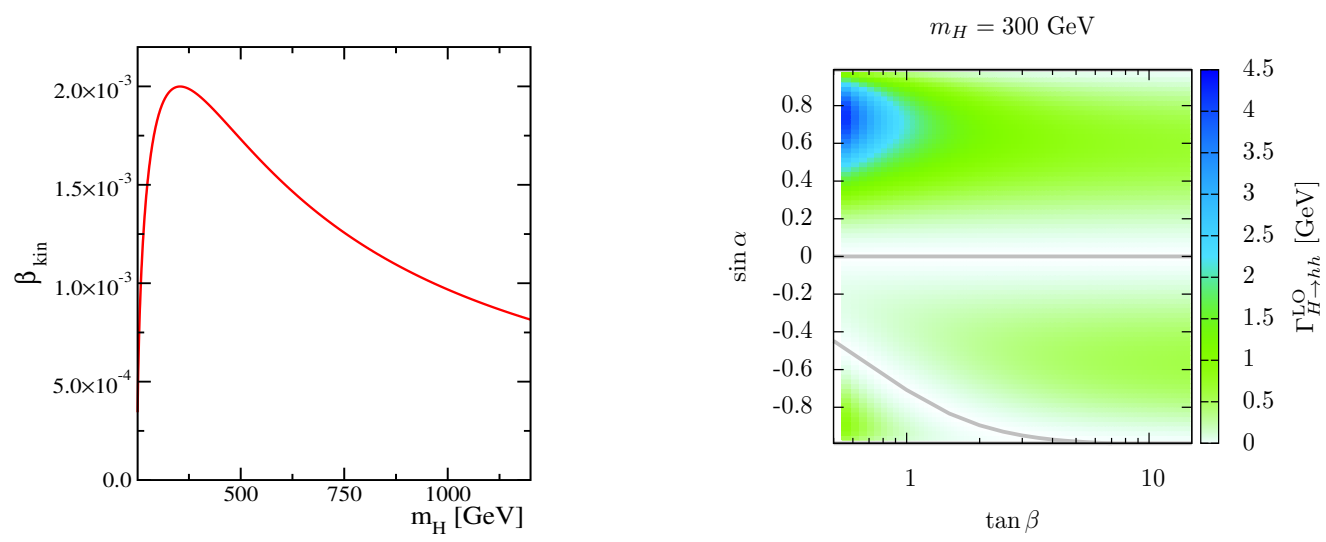

Figure 2. Left panel: kinematical factor $\beta_{\text {kin }}=\sqrt{1-4 m_{h}^{2} / m_{H}^{2}}$ as a function of the heavy Higgs mass, for $m_{h}=125.09 \mathrm{GeV}$. Right panel: leading-order heavy-to-light Higgs decay width $\Gamma_{H \rightarrow h h}^{\mathrm{LO}}$ [in $\mathrm{GeV}$ ] over the $\sin \alpha-\tan \beta$ plane for a fixed heavy Higgs mass of $m_{H}=300 \mathrm{GeV}$. The grey lines signal the configurations $\sin \alpha=0$ and $\tan \beta=-\tan \alpha$ along which $\Gamma_{H \rightarrow h h}^{\mathrm{LO}}$ vanishes.

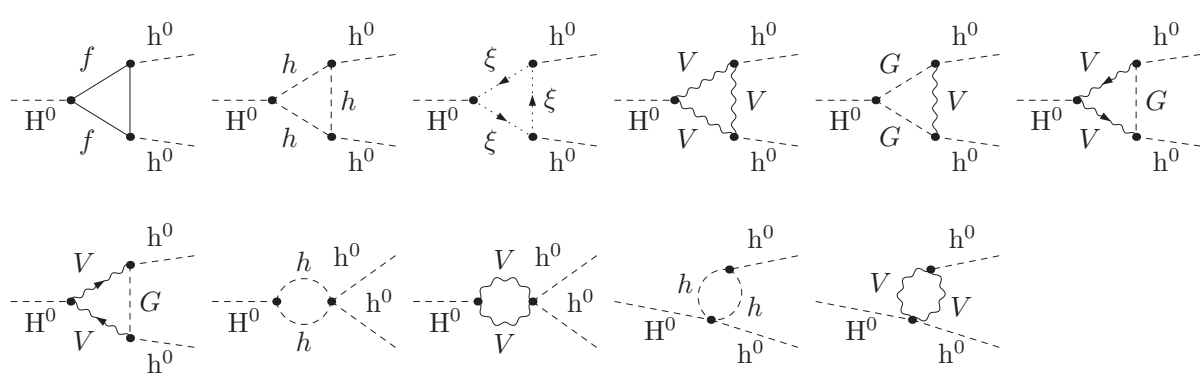

Figure 3. Representative Feynman diagrams for $H \rightarrow h$ at one-loop electroweak accuracy in the 't Hooft-Feynman gauge. The Feynman diagrams are generated using FEYNARTs.STY [127].

\subsection{Electroweak one-loop corrections}

Since all external particles involved in this process are colorless and electrically neutral, the next-to-leading order (NLO) corrections are given by purely weak one-loop effects. These $\mathcal{O}\left(\alpha_{\text {ew }}\right)$ corrections stem from the interference of the LO amplitude and different subsets of one-loop graphs. On the one hand we have the genuine one-particle irreducible (1PI) vertex corrections. These include triangle and bubble-like three-point topologies which involve the exchange of virtual heavy fermions, weak gauge bosons and Higgs bosons, as generically illustrated in figure 3. The neutral Goldstone bosons and the $\mathrm{SU}(2)_{L}$ FaddeevPopov ghost contributions appear explicitly in the 't Hooft-Feynman gauge. In addition to the genuine 1PI topologies, the one-loop corrections involve as well the $H h h$ vertex counterterm, which relies on a combination of Higgs and gauge boson two-point functions, as discussed beforehand in section 3. This contribution cancels the UV-divergent poles of the 1PI amplitude and allows us to write the complete one-loop amplitude in terms of physical (renormalized) parameters. Lastly, we must include the finite wave-function corrections to the external Higgs boson legs (3.31) in the minimal field scheme - while for the on-shell schemes these are identically zero. 
Combining all these pieces we may express the NLO heavy-to-light Higgs decay width as

$$
\Gamma_{H \rightarrow h h}^{\mathrm{NLO}}=\frac{1}{32 \pi m_{H}} \sqrt{1-\frac{4 m_{h}^{2}}{m_{H}^{2}}}\left[\lambda_{H h h}^{2}+2 \operatorname{Re} \lambda_{H h h}\left(\delta \Gamma_{H h h}^{\triangle}+\delta \Gamma_{H h h}^{\mathrm{WF}}+\delta \lambda_{H h h}\right)\right] .
$$

By $\delta \Gamma_{H h h}^{\triangle}$ we denote the one-loop contribution from the 1PI three-point vertex graphs. The wave-function corrections yield

$$
\delta \Gamma_{H h h}^{\mathrm{WF}}=2 \hat{Z}_{h H} \lambda_{H H h}+\hat{Z}_{H h} \lambda_{h h h}=\frac{1}{m_{h}^{2}-m_{H}^{2}}\left[\lambda_{h h h} \hat{\Sigma}_{h H}\left(m_{H}^{2}\right)-2 \lambda_{H H h} \hat{\Sigma}_{h H}\left(m_{h}^{2}\right)\right],
$$

where we have introduced the finite field renormalization constants eq. (3.31) and expanded them to first order in $\alpha_{\text {ew }}$. Finally, $\delta \lambda_{H h h}$ stands for the counterterm of the trilinear scalar coupling. The latter is constructed from the tree-level expression (4.2), expanding all the bare quantities as customary as $X^{0} \rightarrow X+\delta X$. Doing so we find

$$
\begin{aligned}
\delta \lambda_{H h h}= & \lambda_{H h h}\left[\delta Z_{h}+\frac{1}{2} \delta Z_{H}+\frac{1}{2} \frac{\lambda_{h h h}}{\lambda_{H h h}} \delta Z_{h H}+\frac{\lambda_{H H h}}{\lambda_{H h h}} \delta Z_{h H}\right] \\
& +c_{1}^{H h h} \delta m_{h}^{2}+c_{2}^{H h h} \delta m_{H}^{2}+c_{3}^{H h h} \delta m_{h H}^{2}+c_{4}^{H h h} \delta T_{h}+c_{5}^{H h h} \delta T_{H}+c_{6}^{H h h} \frac{\delta v}{v},
\end{aligned}
$$

where the coefficients $c_{i}$ are quoted separately in the appendix.

The relative size of the quantum effects is quantified through the ratio

$$
\delta_{\alpha} \equiv \frac{\Delta \Gamma_{\alpha}^{1-\text { loop }}}{\Gamma_{\alpha}^{\mathrm{LO}}}=\frac{\Gamma_{\alpha}^{\mathrm{NLO}}-\Gamma_{\alpha}^{\mathrm{LO}}}{\Gamma_{\alpha}^{\mathrm{LO}}},
$$

where all quantities are given in the $\alpha_{\mathrm{em}}$-parametrization. The pure one-loop corrections $\Delta \Gamma^{1 \text {-loop }}$ include all terms stemming from the LO-NLO interference.

\section{Phenomenology}

Hereafter we describe the phenomenology of heavy-to-light Higgs decays at NLO EW accuracy. We begin in section 5.2 by completing the discussion on the gauge dependence issues that were pointed out qualitatively in section 3 . We here revisit them on quantitative grounds and justify the choice of the improved on-shell scheme as our default setup for the remainder of the analysis. Furthermore, we perform a dedicated numerical comparison of different schemes and show that these theoretical shortcomings have arguably a negligible impact in practice.

We continue in sections 5.3 and 5.4 with a detailed presentation of our phenomenological analysis. In line with ref. [21], we separately consider two regions of interest, where heavy-to-light Higgs decays are kinematically accessible.

- High-mass region: in which the lighter eigenstate is identified with the discovered SMlike Higgs of (fixed) mass $m_{h}$, while the heavier mass-eigenstate corresponds to an additional heavy Higgs companion with a variable mass $m_{H}$, such that $m_{H}>2 m_{h}$. 
- Low-mass region: where one instead identifies the heavier mass eigenstate with the SM-like Higgs of (fixed) mass $m_{H}$, while $h$ represents now a light Higgs companion and $m_{H}>2 m_{h}$.

Specific scenarios with maximal $H \rightarrow h h$ branching fractions in agreement with all of the model constraints are analysed separately in section 5.5.

\subsection{Computational setup}

In the remainder of our numerical analysis, we fix the SM Higgs boson mass to the best-fit value based on the combined data samples of the ATLAS and CMS experiments $m_{h}=$ $125.09 \mathrm{GeV}$ [65]. Whenever needed, we use in addition the current best averages of the top-quark mass $m_{t}=173.07 \mathrm{GeV}$; the (pole) bottom-quark mass $m_{b}^{\text {pole }}=4.78 \mathrm{GeV}$; and the weak gauge boson masses $m_{\mathrm{W}}=80.385 \mathrm{GeV}, m_{\mathrm{Z}}=91.1875 \mathrm{GeV}$ [102]. The singlet vev is linked to the physical doublet vev through the input parameter $\tan \beta$ as $v_{s}=v_{\text {phys }} \tan \beta$, with $v_{\text {phys }} \equiv v_{G_{F}}=\left(\sqrt{2} G_{F}\right)^{-1 / 2}=246.219 \mathrm{GeV}$. This is in fact equivalent to defining $\tan \beta$ in the $G_{F}$-parametrization. To perform our calculation in the $\alpha_{\mathrm{em}}$-parametrization, we must translate it accordingly through eq. (3.16)

$$
\left.\tan \beta]_{\alpha_{\mathrm{em}}}=\tan \beta\right]_{G_{F}}\left(\frac{v_{\text {phys }}}{v_{\alpha_{\mathrm{em}}}}\right)=\frac{\tan \beta]_{G_{F}}}{\sqrt{1+\Delta r}},
$$

where

$$
v_{\alpha_{\mathrm{em}}}^{2}=\frac{m_{W}^{2}\left(1-m_{W}^{2} / m_{Z}^{2}\right)}{\pi \alpha_{\mathrm{em}}(0)} \quad \text { and hence } \quad v_{\alpha_{\mathrm{em}}}=v_{G_{F}} \sqrt{1+\Delta r} .
$$

Plugging the above relation along with eq. (3.16) into the expression for the decay width (4.4),

$$
\Gamma_{G_{F}}^{\mathrm{LO}}=\Gamma_{\alpha_{\mathrm{em}}}^{\mathrm{LO}}\left(1+\frac{\Delta r}{1+t_{\alpha} / \tan \beta}\right)
$$

which relates the $\alpha_{\mathrm{em}}$ and $G_{F}$ parametrizations up to NLO EW accuracy through

$$
\delta_{G_{F}} \equiv \frac{\Delta \Gamma_{G_{F}}^{1-\text { loop }}}{\Gamma_{G_{F}}^{\mathrm{LO}}}=\delta_{\alpha_{\mathrm{em}}}\left(1-\frac{\Delta r}{1+t_{\alpha} / \tan \beta}\right)+\mathcal{O}\left(G_{F}^{3}\right) .
$$

Feynman rules for the singlet model rely on two independent implementations. For one of them we use LANHEP $[74,75]$ and SLOOPS [15-18] and include a non-linear gauge fixing Lagrangian (2.20). For the second one we generate UFO [128] and FEYNARTs [127] files using FEYNRULES [129], while the counterterms are derived analytically and implemented by hand. Both implementations are in perfect agreement.

The one-loop decay amplitude is generated with FEYNARTS and analytically processed via FormCALC [127]. The loop form factors are handled with dimensional regularization in the 't Hooft-Veltman scheme, and written in terms of standard loop integrals. These are further reduced via Passarino-Veltman decomposition and evaluated with the help of LoopTools [130]. 


\subsection{Scheme choice and gauge invariance}

Gauge-fixing parameters may appear explicitly at intermediate stages in the calculation of $S$-matrix elements in gauge theories. Taken separately, counterterms and unrenormalized loop amplitudes may in general depend on the gauge-fixing parameters and are eventually also UV-divergent. We only expect these UV divergent contributions to cancel once all the different building blocks are combined together into predictions for physical observables. Nonetheless, depending on which renormalization conditions are chosen for a certain input parameter $X$, one may obtain loop amplitudes which, albeit finite, still depend on the gauge-fixing. These situations reflect that, for such a renormalization scheme, the definition for $X$ is gauge-dependent.

In the following we check the different renormalization schemes introduced in section 3.3.6 in the light of gauge independence. We compute the one-loop correction to the heavy-to-light Higgs decay width $\delta \Gamma_{H \rightarrow h h}^{1-\text { loop }} \equiv \Gamma_{H \rightarrow h h}^{\mathrm{NLO}}-\Gamma_{H \rightarrow h h}^{\mathrm{LO}}$ in the general nonlinear gauge of eq. (2.20), where the quantities $\Gamma_{H \rightarrow h h}^{\mathrm{LO}}$ and $\Gamma_{H \rightarrow h h}^{\mathrm{NLO}}$ are given by eqs. (4.1) and (4.4) respectively. We resort to the SloopS implementation of the singlet model Feynman rules which includes the general non-linear gauge-fixing Lagrangian of eq. (2.20), and vary the gauge-fixing parameters $\{$ nlgs $\}=\left\{\tilde{\alpha}, \tilde{\beta}, \tilde{\kappa}, \tilde{\delta}_{1}, \tilde{\delta}_{2}, \tilde{\epsilon}_{1}, \tilde{\epsilon}_{2}\right\}$ within the fiducial range $\{$ nlgs $\}=0 \ldots 10$. Notice that the lower endpoint $\{$ nlgs $=0\}$ reproduces the familiar 't Hooft-Feynman linear gauge. As a sample parameter space point we take

$$
m_{h}=125.09 \mathrm{GeV}, \quad m_{H}=260 \mathrm{GeV}, \quad \sin \alpha=0.3, \quad \tan \beta=5,
$$

which gives a leading-order width $\Gamma_{H \rightarrow h h}^{\mathrm{LO}}=0.137 \mathrm{GeV}$. In table 4 we compare the results for $\delta \Gamma_{H \rightarrow h h}^{1-l o o p}$ in the linear gauge $(\{n \operatorname{lgs}=0\})$ and one exemplary non-linear setup $(\{$ nlgs $=10\})$. Simultaneously, we check the UV-finiteness of our results by sweeping the range $\Delta=0 \ldots 10^{7}$, where the parameter $\Delta$ trades the UV-divergences of the one-loop amplitude as defined by eq. (3.38). Gauge independence and UV-finiteness are verified if $\delta \Gamma^{1 \text {-loop }}$ remains unchanged (within numerical precision) under these varations. ${ }^{9}$ The fact that in the first two columns $\delta \Gamma^{1-\text { loop }}$ remain constant confirms that all of the four schemes introduced in section 3.3.6 yield UV-finite results in the linear gauge. However, only the mixed $\overline{\mathrm{MS}} / \mathrm{OS}$ and the improved OS setups produce UV-finite, $\{$ nlgs $\}$-independent results for the generalized non-linear gauge-fixing. Instead, in the minimal field and the OS schemes we observe left-over $\delta \Gamma^{1-\text { loop }}$ dependencies on the gauge-fixing parameters. These $\{$ nlgs $\}$-dependent remainders affect both the finite parts and the UV-divergent contributions, and are thus responsible for the incomplete cancellation of the UV-poles, cf. the last column of table 4. This breakdown can be ultimately traced back to the renormalization condition that determines the mixed mass counterterm $\delta m_{h H}^{2}$. Its definitions in the minimal field scheme (3.30) and the OS scheme (3.35) are not gauge-independent, and lead to a $\{$ nlgs $\}$-dependent decay width. Instead, we find no residual $\{$ nlgs $\}$-dependencies in the mixed $\overline{\mathrm{MS}} / \mathrm{OS}$ and the improved OS schemes, in which $\delta m_{h H}^{2}$ is fixed via the gaugeindependent definitions of eq. (3.41) and (3.42) respectively. We make these observations

\footnotetext{
${ }^{9}$ Using double precision we expect an agreement of 14 to 15 digits. Given the variation ranges $\Delta=$ $0 \ldots 10^{7}$ and $\{\mathrm{nlgs}\}=0 \ldots 10$, we deem the test as satisfactory if 6 to 8 common digits are achieved.
} 


\begin{tabular}{|l|c|c|c|}
\cline { 2 - 4 } \multicolumn{1}{c|}{} & \multicolumn{3}{c|}{$\delta \Gamma_{H \rightarrow h h}^{1-\text { loop }}[\mathrm{GeV}]$} \\
\hline Scheme & $\Delta=0,\{\mathrm{nlgs}\}=0$ & $\Delta=10^{7},\{\mathrm{nlgs}\}=0$ & $\Delta=10^{7},\{\mathrm{nlgs}\}=10$ \\
\hline Minimal field & $+4.28079888 \times 10^{-3}$ & $+4.2807988 \mathbf{2} \times 10^{-3}$ & $-6.63340412 \times 10^{4}$ \\
OS & $+4.26334888 \times 10^{-3}$ & $+4.26334886 \times 10^{-3}$ & $-5.27015844 \times 10^{3}$ \\
Mixed MS $/ O S$ & $+6.8467506 \times 10^{-3}$ & $+6.8467504 \times 10^{-3}$ & $+6.8467500 \times 10^{-3}$ \\
Improved OS & $+3.9393569 \times 10^{-3}$ & $+3.9393568 \times 10^{-3}$ & $+3.9393556 \times 10^{-3}$ \\
\hline
\end{tabular}

Table 4. Checks on UV-finiteness and gauge independence of the one-loop correction to the heavy-to-light Higgs decay width $\delta \Gamma_{H \rightarrow h h}^{1-l o o p}$ (in $\mathrm{GeV}$ ) within the different renormalization schemes introduced in section 3.3.6. The model parameters are fixed as in eq. (5.5). For the (scale dependent) minimal field scheme we set the renormalization scale at $\mu_{R}^{2}=\left(m_{h}^{2}+m_{H}^{2}\right) / 2$. Bold-faced numbers highlight the first departing digits between the entries of the different columns in a given row.

patent in table 5, where we display the numerical value of the mixing counterterm $\delta m_{h H}^{2}$ corresponding to the four renormalization schemes under analysis. Since the counterterm is not UV finite, we split it into a finite and singular part as (with $\Delta$ as defined in (3.38))

$$
\delta m_{h H}^{2}=\left.\delta m_{h H}^{2}\right|^{\infty} \cdot \Delta+\left.\delta m_{h H}^{2}\right|^{\text {fin }} .
$$

Neither the coefficient of the UV pole $\left.\delta m_{h H}^{2}\right|^{\infty}$ nor the finite remainder $\left.\delta m_{h H}^{2}\right|^{\text {fin }}$ depend on the gauge-fixing parameters when we fix $\delta m_{h H}^{2}$ either in the mixed $\overline{\mathrm{MS}} / \mathrm{OS}$ or the improved OS conditions. Instead, both terms are shifted when we switch from the linear $\{$ nlgs $\}=0$ to the non-linear gauge-fixing choice $\{$ nlgs $\}=10$, when the calculation is performed using the minimal field or the OS schemes. In view of the fact that $\delta m_{h H}^{2}$ (along with the mixed field renormalization $\delta Z_{h H}$, cf. table 3 ) are the only different ingredients between these four schemes, they are ultimately responsible for the finite $\{n \operatorname{lgs}\}$-dependent remainders in $\delta \Gamma^{1 \text {-loop }}$ in the latter two schemes - and linked to them, of the uncancelled UV poles. We emphasize as well that these concomitant UV divergences vanish for $\{$ nlgs $\}=0$ and hence do not feature in the customary 't Hooft-Feynman gauge, where the results in all schemes are UV finite. Finally, let us also notice that, given the relation between the mixed mass counterterm and the mixing angle via eq. (3.24), a gauge-independent $\delta m_{h H}^{2}$ supports a more physical interpretation of the mixing angle, viz. as value that could be extracted from e.g. a deviation in the LHC Higgs signal strengths or, alternatively, an excess which points to the direct production of the heavy scalar. ${ }^{10}$

For practical purposes, therefore, the proven robustness of the improved OS scheme (giving in all cases UV-finite, \{nlgs $\}$-independent, and numerically stable renormalized one-loop amplitudes) justifies its use as default scheme choice in our numerical analysis hereafter. Moreover, the excellent agreement between the $\delta \Gamma^{1-\text { loop }}$ results for the different schemes in the linear 't Hooft-Feynman gauge - as explicitly shown further down - give convincing arguments that also the schemes where the mixed mass counterterm is gauge

\footnotetext{
${ }^{10}$ Similar lines of argument are used in the context of the renormalization of the $\tan \beta$ parameter in the MSSM, cf. e.g. table 2 in ref. [17].
} 


\begin{tabular}{|l|c|c|l|c|c|}
\hline$\left.\delta m_{h H}^{2}\right|^{\infty}$ & $\{\mathrm{nlgs}\}=0$ & $\{\mathrm{nlgs}\}=10$ & $\left.\delta m_{h H}^{2}\right|^{\text {fin }}$ & $\{\mathrm{nlgs}\}=0$ & $\{\mathrm{nlgs}\}=10$ \\
\hline Minimal field & $-5.80 \times 10^{2}$ & $-9.44 \times 10^{2}$ & Minimal field & $+5.72 \times 10^{3}$ & $+8.48 \times 10^{3}$ \\
OS & $-5.80 \times 10^{2}$ & $-9.44 \times 10^{2}$ & OS & $+5.75 \times 10^{3}$ & $+8.80 \times 10^{3}$ \\
Mixed MS/OS & $-5.80 \times 10^{2}$ & $-5.80 \times 10^{2}$ & Mixed MS/OS & $-2.48 \times 10^{2}$ & $-2.48 \times 10^{2}$ \\
Improved OS & $-5.80 \times 10^{2}$ & $-5.80 \times 10^{2}$ & Improved OS & $+5.72 \times 10^{3}$ & $+5.72 \times 10^{3}$ \\
\hline
\end{tabular}

Table 5. Dependence on the gauge-fixing parameters of the mixed mass counterterm $\delta m_{h H}^{2}$ (in $\mathrm{GeV}^{2}$ ) within the different renormalization schemes introduced in section 3.3.6. The model parameters are fixed as in eq. (5.5). For the (scale dependent) minimal field scheme we set the renormalization scale at $\mu_{R}^{2}=\left(m_{h}^{2}+m_{H}^{2}\right) / 2$.

dependent render reliable results - at least as long as the linear 't Hooft-Feynman gauge is used and, in the case of the minimal field scheme the renormalization scale is chosen in the ballpark of the relevant physical scales. This is again in line with analogue situations such as e.g. the squark sector of the MSSM [18, 121, 122].

\subsection{High-mass region}

In table 6 we evaluate $\Gamma_{H \rightarrow h h}^{\mathrm{LO}}$ and $\Gamma_{H \rightarrow h h}^{\mathrm{NLO}}$ for representative parameter choices and different renormalization schemes. The relative one-loop EW corrections are given in both the $\alpha_{\mathrm{em}}{ }^{-}$ parametrization and the $G_{F}$-parametrization introduced in section 4. Our results show decay rates that strongly vary with the relevant parameters of the model. The heavy-tolight Higgs decay width significantly depends on the decaying Higgs mass $m_{H}$, changing by two orders of magnitude when sweeping the range $m_{H}=300 \ldots 700 \mathrm{GeV}$. For heavy Higgs masses close to the di-Higgs threshold, the partial Higgs widths lie in the ballpark of $\mathcal{O}(0.01-0.1) \mathrm{GeV}$. These results depend as well on the mixing angle, and change by roughly one order of magnitude from small (viz. $\sin \alpha \simeq 0.1$ ) to moderate mixing angles (viz. $\sin \alpha \simeq 0.3$ ). For larger heavy Higgs masses, the $\Gamma_{H \rightarrow h h}^{\mathrm{NLO}}$ values may rise up to the few $\mathrm{GeV}$ level. The mild numerical discrepancies between the different schemes are indicative of small theoretical uncertainties in the 't Hooft-Feynman gauge. For a more general gauge-fixing choice, though, the minimal field and on-shell schemes are no longer reliable, in view of their proven gauge-dependent nature. It is also worth noticing that the radiative corrections in the $G_{F}$-parametrization $\left(\delta_{G_{F}}\right)$ are generically smaller than in the $\alpha_{\mathrm{em}}$-parametrization. The reason is twofold: i) part of the NLO EW corrections in the latter case $\left(\delta_{\alpha}\right)$ are contained in the $\Delta r$ parameter, and hence already embedded into the $G_{F}$-scheme LO calculation (cf. eq. (5.3)). Consequently, the quantum effects encoded by $\Delta r$ do not belong to $\delta_{G_{F}}$; ii) for phenomenologically relevant scenarios, $\Delta r$ is dominated by purely SM effects, for which $\Delta^{\mathrm{SM}}>0[98,103-107]$, and thereby $\delta_{\alpha}>\delta_{G_{F}}$, given the relation between both (5.4).

The analysis is complemented in figure 4 with a thorough survey of the parameter space dependencies. The NLO results are calculated in the improved OS scheme. The shaded regions are ruled out by different theoretical and experimental constraints on the model: i) the ranges $m_{H}>840 \mathrm{GeV}$ (left panel) and $\tan \beta<1.27$ (right panel) are excluded 


\begin{tabular}{|c|c|c|c|c|c|c|}
\hline$m_{H}[\mathrm{GeV}]$ & $\sin \alpha$ & $\Gamma_{\alpha}^{\mathrm{LO}}(H \rightarrow h h)[\mathrm{GeV}]$ & & $\Gamma_{\alpha}^{\mathrm{NLO}}(H \rightarrow h h)[\mathrm{GeV}]$ & $\delta_{\alpha}[\%]$ & $\delta_{G_{F}}[\%]$ \\
\hline \multicolumn{7}{|c|}{$\tan \beta=5$} \\
\hline \multirow{9}{*}{300} & \multirow{3}{*}{0.1} & \multirow{3}{*}{$4.374 \times 10^{-2}$} & OS & $4.516 \times 10^{-2}$ & 3.250 & 3.130 \\
\hline & & & Improved OS & $4.509 \times 10^{-2}$ & 3.106 & 2.990 \\
\hline & & & Minimal field & $4.544 \times 10^{-2}$ & 3.895 & 3.751 \\
\hline & \multirow{3}{*}{0.2} & \multirow{3}{*}{0.171} & OS & 0.177 & 3.371 & 3.248 \\
\hline & & & Improved OS & 0.177 & 3.218 & 3.100 \\
\hline & & & Minimal field & 0.178 & 4.033 & 3.886 \\
\hline & \multirow{3}{*}{0.3} & \multirow{3}{*}{0.362} & OS & 0.375 & 3.583 & 3.455 \\
\hline & & & Improved OS & 0.374 & 3.400 & 3.278 \\
\hline & & & Minimal field & 0.377 & 4.281 & 4.127 \\
\hline \multirow{9}{*}{500} & \multirow{3}{*}{0.1} & \multirow{3}{*}{0.221} & OS & 0.234 & 5.667 & 5.456 \\
\hline & & & Improved OS & 0.233 & 5.438 & 5.236 \\
\hline & & & Minimal field & 0.237 & 6.989 & 6.730 \\
\hline & \multirow{3}{*}{0.2} & \multirow{3}{*}{0.868} & OS & 0.920 & 5.980 & 5.761 \\
\hline & & & Improved OS & 0.917 & 5.728 & 5.518 \\
\hline & & & Minimal field & 0.932 & 7.441 & 7.168 \\
\hline & \multirow{3}{*}{0.3} & \multirow{3}{*}{1.831} & OS & 1.951 & 6.566 & 6.329 \\
\hline & & & Improved OS & 1.945 & 6.237 & 6.012 \\
\hline & & & Minimal field & 1.983 & 8.294 & 7.995 \\
\hline \multirow{9}{*}{700} & \multirow{3}{*}{0.1} & \multirow{3}{*}{0.586} & OS & 0.597 & 1.948 & 1.876 \\
\hline & & & Improved OS & 0.601 & 2.569 & 2.473 \\
\hline & & & Minimal field & 0.598 & 2.009 & 1.935 \\
\hline & \multirow{3}{*}{0.2} & \multirow{3}{*}{2.296} & OS & 2.355 & 2.583 & 2.489 \\
\hline & & & Improved OS & 2.369 & 3.188 & 3.071 \\
\hline & & & Minimal field & 2.366 & 3.056 & 2.944 \\
\hline & \multirow{3}{*}{0.3} & \multirow{3}{*}{4.845} & OS & 5.026 & 3.742 & 3.606 \\
\hline & & & Improved OS & 5.056 & 4.353 & 4.195 \\
\hline & & & Minimal field & 5.082 & 4.893 & 4.716 \\
\hline
\end{tabular}

Table 6. Heavy-to-light Higgs decay width $\Gamma_{H \rightarrow h h}$ at LO and NLO EW accuracy, for representative parameter choices and different renormalization schemes, in the high-mass region. The total decay widths are obtained in the $\alpha_{\mathrm{em}}$-parametrization, as defined in eqs. (4.7), while the relative oneloop EW effects are quantified in both the $\alpha_{\mathrm{em}}$-parametrization and the $G_{F}$-parametrization, cf. eq. (5.4). For the (scale-dependent) minimal field scheme, the renormalization scale is fixed to the geometrical average mass $\mu_{R}^{2}=p_{*}^{2}=\left(m_{h}^{2}+m_{H}^{2}\right) / 2$. The input value for $\tan \beta$ is linked to the singlet vev through $v_{s}=\left(\sqrt{2} G_{F}\right)^{-1 / 2} \tan \beta$.

by perturbativity ii) $|\sin \alpha|>0.31$ (central panel, green shading) is incompatible with electroweak constraints from the $m_{W}$ measurement; finally, the central range $|\sin \alpha|<0.06$ (central panel, orange shading) is incompatible with vacuum stability.

Most features observed in figure 4 can be readily traced back to the LO dynamics which governs the decay process. The two key players, as alluded to above, are the trilinear Higgs self-coupling $\lambda_{H h h}$ and the characteristic $1 \rightarrow 2$ kinematics. The former is responsible for the quadratic growth $\Gamma_{H \rightarrow h h} \sim \mathcal{O}\left(m_{H}^{2}\right.$ ) (cf. eq. (A.2)), which overcomes the phase space suppression at $m_{H}^{2} \gg m_{h}^{2}$, and explains the power-like increase as a function of $m_{H}$ (left 

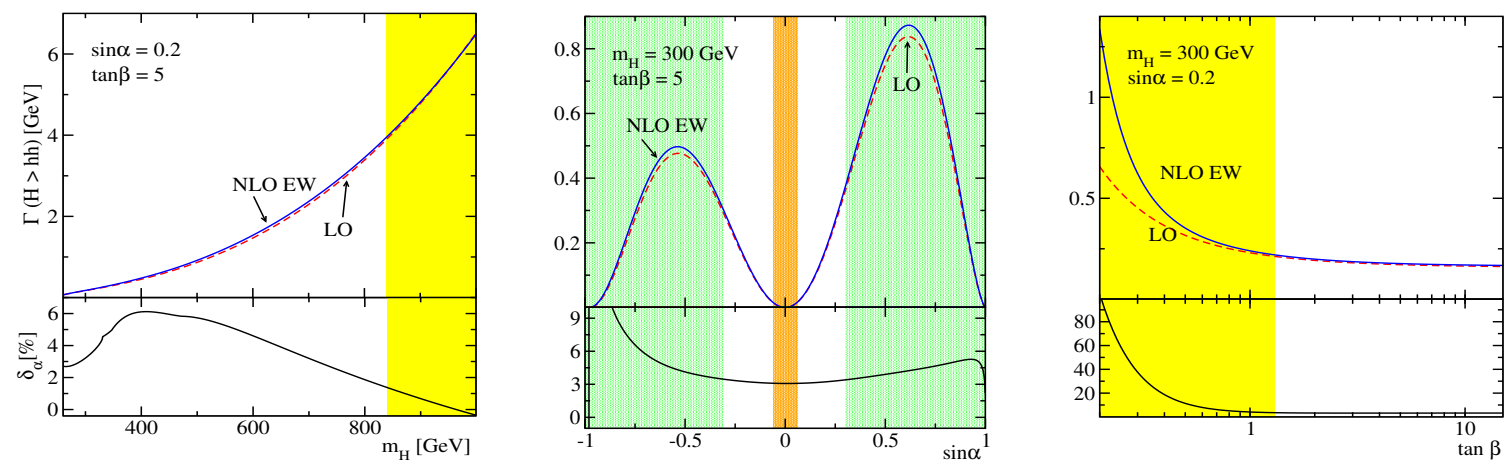

Figure 4. Heavy-to-light Higgs decay width $\Gamma_{H \rightarrow h h}$ in the high-mass region. The results are shown at LO (dashed, red) and NLO (full, blue) as a function of the relevant parameters of the model. The lower subpannels show the relative one-loop EW correction in the $\alpha_{\text {em-parametrization }}$ (4.7). Renormalization is performed in the improved on-shell scheme. The shaded regions are excluded by constraints (see the text for more details).

panel in figure 4). The NLO-corrected result with respect to the mixing angle mimics the LO result, with the expected nodes in the decoupling limits $|\sin \alpha|=0$ or 1 as well as for $\tan \beta=-\tan \alpha$ (cf. the central panel of figure 4).

Unlike the stark changes observed for the decay width, the relative one-loop EW corrections are much more stable, positive, and of the order of few percent. Differences between the $\alpha_{\mathrm{em}}$-parametrization and the $G_{F}$-parametrization, as well as between the different renormalization schemes, are mild and remain typically below the percent level.

The slight kink in $\delta_{\alpha}$ for $m_{H} \simeq 350 \mathrm{GeV}$ (left panel, figure 4) reflects the top-quark threshold. The finite correction $\delta_{\alpha} \sim 3 \%$ for $\sin \alpha \rightarrow 0$ (cf. the lower subpanel of figure 4, center) follows from the fact that both $\Gamma_{H \rightarrow h h}^{\mathrm{LO}}$ and $\Gamma_{H \rightarrow h h}^{\mathrm{NLO}}$ tend to zero in this limit, while its ratio remains roughly constant. The unphysical large effects at $\sin \alpha \lesssim-0.9$ are due to the LO node in the limit $\tan \alpha \rightarrow-\tan \beta$, for which $\lambda_{H h h}=0$. The pronounced NLO slope at low $\tan \beta$ is ultimately due to the exchange of virtual Higgs bosons, and constitutes a telltale imprint of the singlet model dynamics at the one-loop level. While the fermion and the gauge boson-mediated contributions are all controlled by (globally rescaled) gauge couplings, the size of the Higgs-mediated loops is governed by the Higgs self-couplings. These are strongly enhanced for $\tan \beta \ll 1$, specially the Higgs boson two-point graphs, which depend on them quadratically. For low enough $\tan \beta$ values, e.g. typically $\tan \beta \lesssim 0.3$ and for $m_{H} \gtrsim 300 \mathrm{GeV}$, the relative yield $\delta_{\alpha}$ exceeds $\sim 50 \%$, indicating that the process becomes effectively loop-induced. Such sizable loop effects are nonetheless hampered in practice, owing to the unitarity and perturbativity bounds which severely constrain the phenomenologically viable low-tan $\beta$ range. The limit $\tan \beta \ll 1$ corresponds in fact to the onset of a strongly-coupled regime, in which at least one of the scalar self-couplings becomes non-perturbative, cf. also the discussion in section 2.6.

Complementary vistas to the $H \rightarrow h$ landscape are displayed in figure 5 . Here we show the relative one-loop effects $\delta_{\alpha}$ (4.7) as density contours in the $\sin \alpha-\tan \beta$ plane. The yellow contour separates the allowed and excluded regions in the parameter space. 

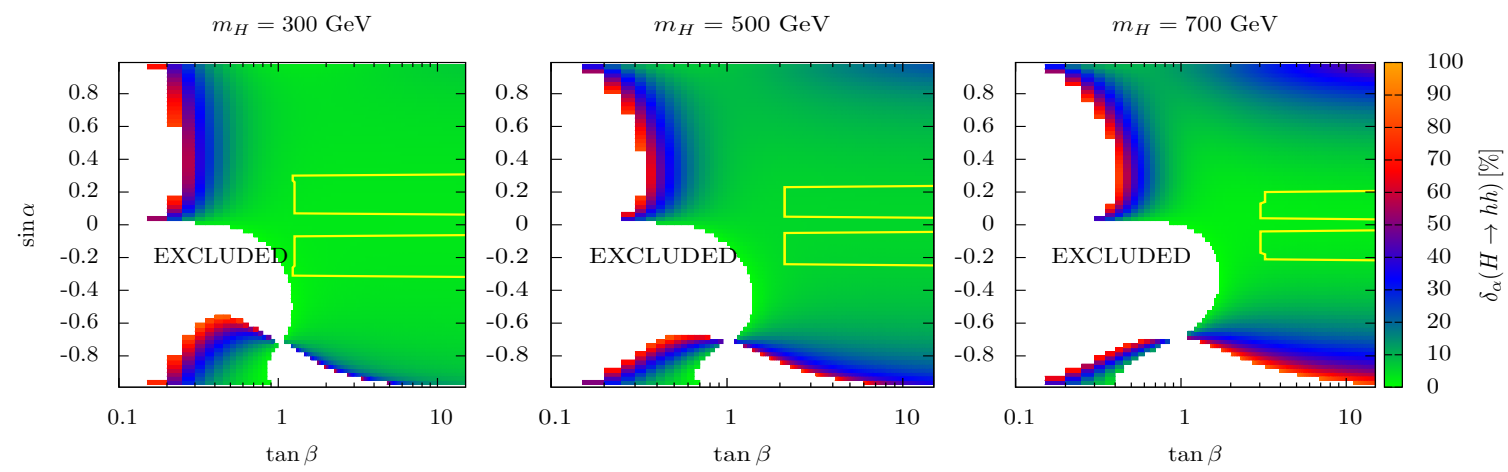

Figure 5. Relative one-loop EW corrections in the $\alpha_{\mathrm{em}}$-parametrization (4.7), projected on the $\sin \alpha-\tan \beta$ plane for exemplary heavy Higgs masses in the high-mass region. The white voids correspond to regions with $\delta_{\alpha} \gtrsim 100 \%$. Renormalization is performed in the improved on-shell scheme. The yellow contour separates the allowed and excluded regions in the parameter space.
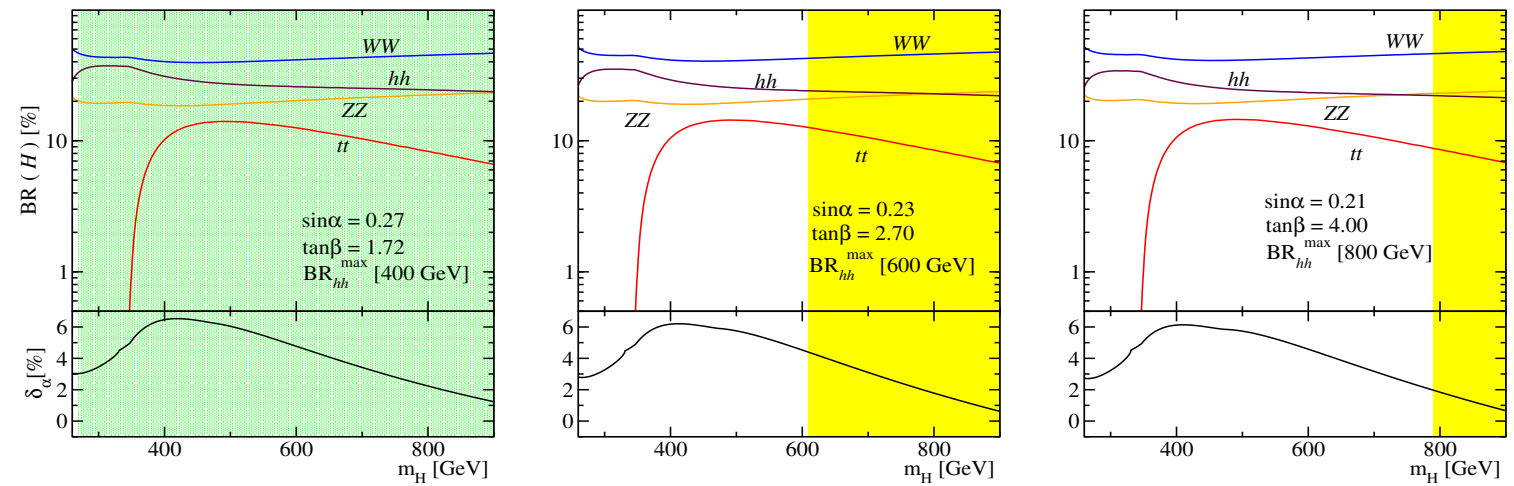

Figure 6. Heavy Higgs branching ratios (in \%) as a function of the heavy Higgs mass in the highmass region. The mixing angle and $\tan \beta$ values are fixed in each panel such that they maximize the $H \rightarrow h h$ branching ratio at LO for a given heavy Higgs mass of 400 (left), 600 (center) and $800 \mathrm{GeV}$ (right) [90]. Decay modes into light fermions and loop-induced decays into gauge bosons lie below $\mathcal{O}(0.1) \%$ and are not shown. The lower subpanels show the relative one-loop EW correction $\left(\alpha_{\mathrm{em}}\right.$ parametrization (4.7)) to $\Gamma_{H \rightarrow h h}^{\mathrm{LO}}$ for the same parameter variations. The shaded (green) area in the left plot is ruled out by the W-mass measurement. Excluded regions in the central and right panels (in yellow) are incompatible with perturbativity and $m_{W}$.

Only the horizontal fringes enclosed by the contour are compatible with all constraints on the model. The white voids stand for values of $\delta_{\alpha} \gtrsim 100 \%$ and correspond to regions where $\delta_{\alpha}$ is no longer a meaningful measure of the relative quantum effects, while it instead indicates that the decay process becomes loop-induced. We find this situation: i) along the strip $\tan \alpha \simeq-\tan \beta$, due to the suppressed tree-level couplings; and ii) for $\tan \beta<1$, due to the $\cot \beta$-enhanced Higgs-mediated loops.

The impact of heavy-to-light Higgs decays on the decay pattern of the heavy Higgs state is portrayed in figure 6 . The branching ratios for the leading decay channels are represented as a function of the heavy Higgs mass. The mixing angle and $\tan \beta$ values are fixed in each panel such that they maximize the $H \rightarrow h h$ branching ratio for a given heavy 

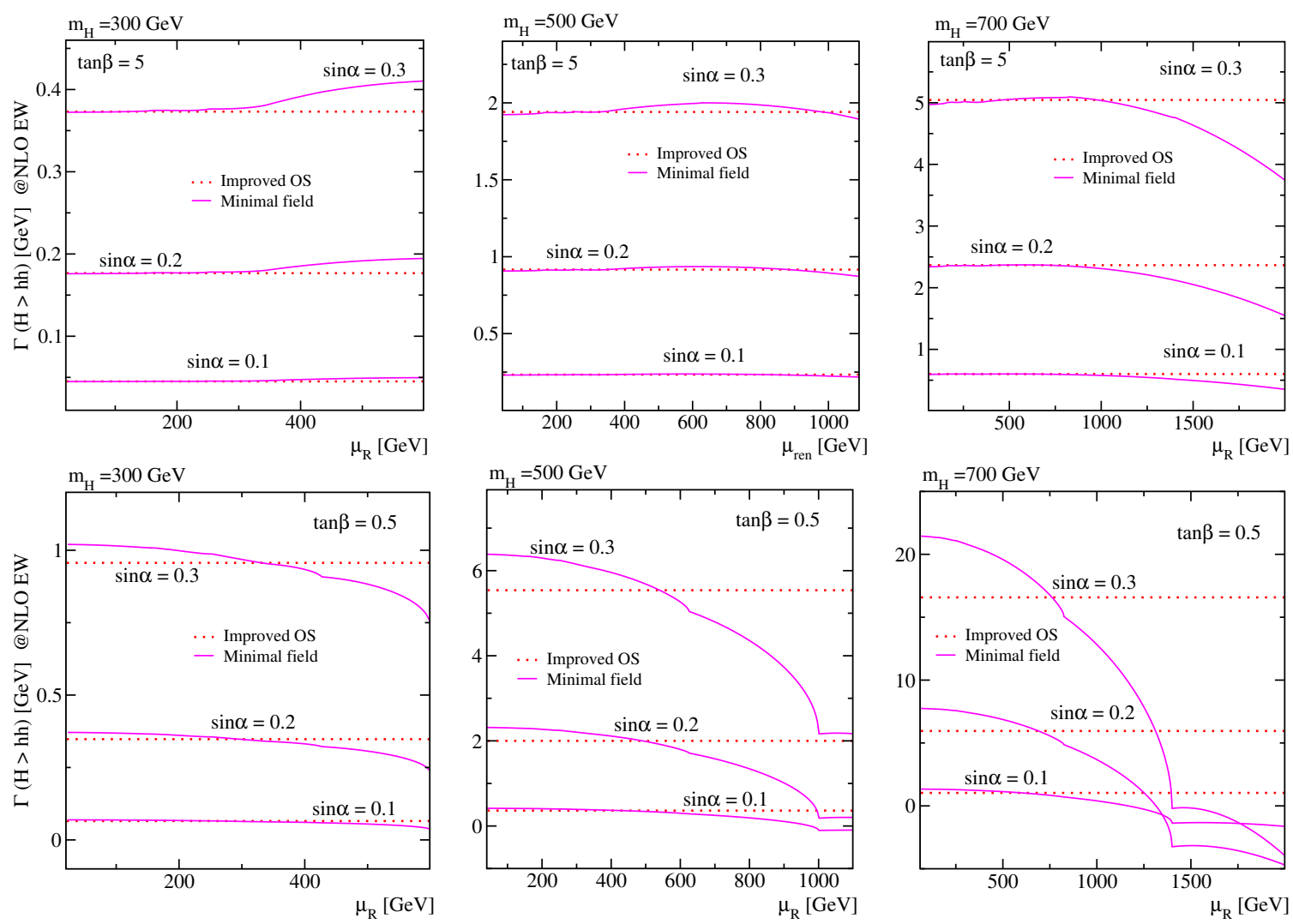

Figure 7. NLO decay width $\Gamma_{H \rightarrow h h}^{\mathrm{NLO}}$ as a function of the renormalization scale in the high-mass region, for exemplary heavy Higgs masses, mixing angles, and $\tan \beta$ choices. The scale-dependent predictions for the minimal field scheme are represented by the solid (magenta) lines. The scaleindependent reference value (dotted, red lines) we obtain in the improved OS scheme. Parameter space constraints are not shown.

Higgs mass [90], as explicitly indicated in the figure. In this plot, we show the partial decay widths to SM fields by rescaling the SM predictions [44], while for the $H \rightarrow h h$ we use the LO result. ${ }^{11}$ As well known, bosonic modes dominate the Higgs boson decays at high masses [7-9]. We find a rather featureless profile, with WW being the leading mode and with roughly no changes over the whole mass range. Only the decays into top-quark pairs are also competitive, and attain up to $\mathrm{BR} \sim \mathcal{O}(10) \%$. The remaining fermionic channels, as well as the loop-induced $\gamma \gamma, \gamma \mathrm{Z}$ and $g g$ modes, stagnate at the $\mathcal{O}(0.1) \%$ level or below and are not shown. In the lower subpanels we show the relative one-loop EW correction to the heavy-to-light Higgs decay width for the same parameter variation.

Finally, in figure 7 we analyse how $\Gamma_{H \rightarrow h h}^{\mathrm{NLO}}$ varies with the renormalization scale in the (scale-dependent) minimal field scheme, as introduced in eq. (3.30). We compare the minimal field to the (scale-independent) improved OS scheme, which we show as reference value. For $\tan \beta=5, \Gamma_{H \rightarrow h h}^{N L O}$ flattens not far from the geometrical average mass scale $\mu_{R}^{2} \simeq p_{*}^{2}=\left(m_{h}^{2}+m_{H}^{2}\right) / 2$. Precisely around this value, both the minimal field and the

\footnotetext{
${ }^{11}$ A global study including all Higgs decays in the singlet model to state-of-the-art accuracy lies beyond the scope of the present study and will be discussed in a forthcoming publication [131].
} 
improved OS predictions tend to converge, suggesting that $\mu_{R}^{2}=p_{*}^{2}$ in eq. (3.30) is indeed a convenient scale choice for the former. Moreover, the very stable NLO predictions around this scale, added up to the mild changes with the different renormalization schemes shown in table 6 , indicate a small theoretical uncertainty.

Much steeper scale variations arise instead in the $\tan \beta<1$ region. Here, the $\Gamma_{H \rightarrow h h}^{\mathrm{NLO}}$ predictions become unstable, especially for heavy Higgs masses. Such instability may be once more traced back to the Higgs-mediated scalar two-point graphs: these become overly large owing to the enhanced Higgs self-couplings, and artificially dominate the scale dependence in these regions. Such a stark scale dependence is simply the reflect of the poor perturbative behavior of the model in the vicinity of a strongly-coupled regime $\lambda \sim \mathcal{O}(4 \pi)$, which obviously translates into a huge theoretical uncertainty.

\subsection{Low-mass region}

Assuming now $m_{H}=125.09 \mathrm{GeV}$ and a free light Higgs mass $m_{h}$, direct LEP and LHC mass bounds, and most remarkably the measured LHC Higgs signal strengths, narrow the viable $\sin \alpha$ region down to a slim fringe $|\sin \alpha| \lesssim 1$. Constraints become particularly tight in the region of interest $m_{H}>2 m_{h}$, given the limited tolerable room for deviations in the total SM-like Higgs width when additional decay modes feature. State-of-the-art LHC constraints on the total Higgs width place an upper limit of $\Gamma_{h} \leq 22 \mathrm{MeV}[132,133]$. For definiteness, we hereafter adopt the fiducial choice $\sin \alpha=0.998$ [21].

In figure 8 we examine the parameter space dependence of $\Gamma_{H \rightarrow h h}$ in this scenario. Complementarily, in table 7 we provide precise predictions for specific parameter space points, while comparing again the different renormalization schemes. In figure 9 we analyse the $m_{h}-\tan \beta$ interplay by showing the total NLO amplitude $\Gamma_{H \rightarrow h h}^{\mathrm{NLO}}(4.4)$ and the relative NLO correction $\delta_{\alpha}$ (4.7) in the form of two-dimensional density maps.

The obtained $\Gamma_{H \rightarrow h h}^{\mathrm{NLO}}$ values span two orders of magnitude, ranging from $\mathcal{O}\left(10^{-3}\right)$ down to $\mathcal{O}\left(10^{-5}\right) \mathrm{GeV}$ as we navigate throughout the different parameter space regions. This sharp variation is again connected to the behavior of the leading-order coupling $\lambda_{H h h}$ : whilst it tends to zero in the limit $\sin \alpha \rightarrow 1$, it can yet contribute if the $\cot \beta$-enhanced terms are large enough. Either way, let us once more recall that a significant patch of the low-tan $\beta$ range is in practice precluded by the different constraints on the model (see e.g. the top panels of figure 8 and figure 9 ). In particular, the shaded regions at small $\tan \beta$ and low Higgs masses are incompatible with the LHC Higgs signal strength measurements. Another salient feature is the steep rise of the quantum corrections at low $\tan \beta$ (see the top panels of figure 8): these are positive, tend to increase with the light Higgs mass, and may surmount the $\mathcal{O}(50 \%)$ level. Aside from the discussed Higgs-mediated loop enhancements, additional mechanisms reinforce this behavior in this case: i) the suppressed tree-level decay amplitude, due to the lesser phase space available, the closer we move to the kinematical threshold; ii) the vicinity of the di-Higgs loop threshold, which invigorates the light Higgsmediated loops even further. For $\tan \beta>1$ the corrections are instead moderate and negative, becoming even more so for very light $m_{h}$ values. The latter effect may be traced back to the fermionic (viz. the top-mediated) three-point loops, which are in this case the dominant source of quantum corrections and present a trademark logarithmic dependence $\sim \log \left(m_{t}^{2} / m_{h}^{2}\right)$. 

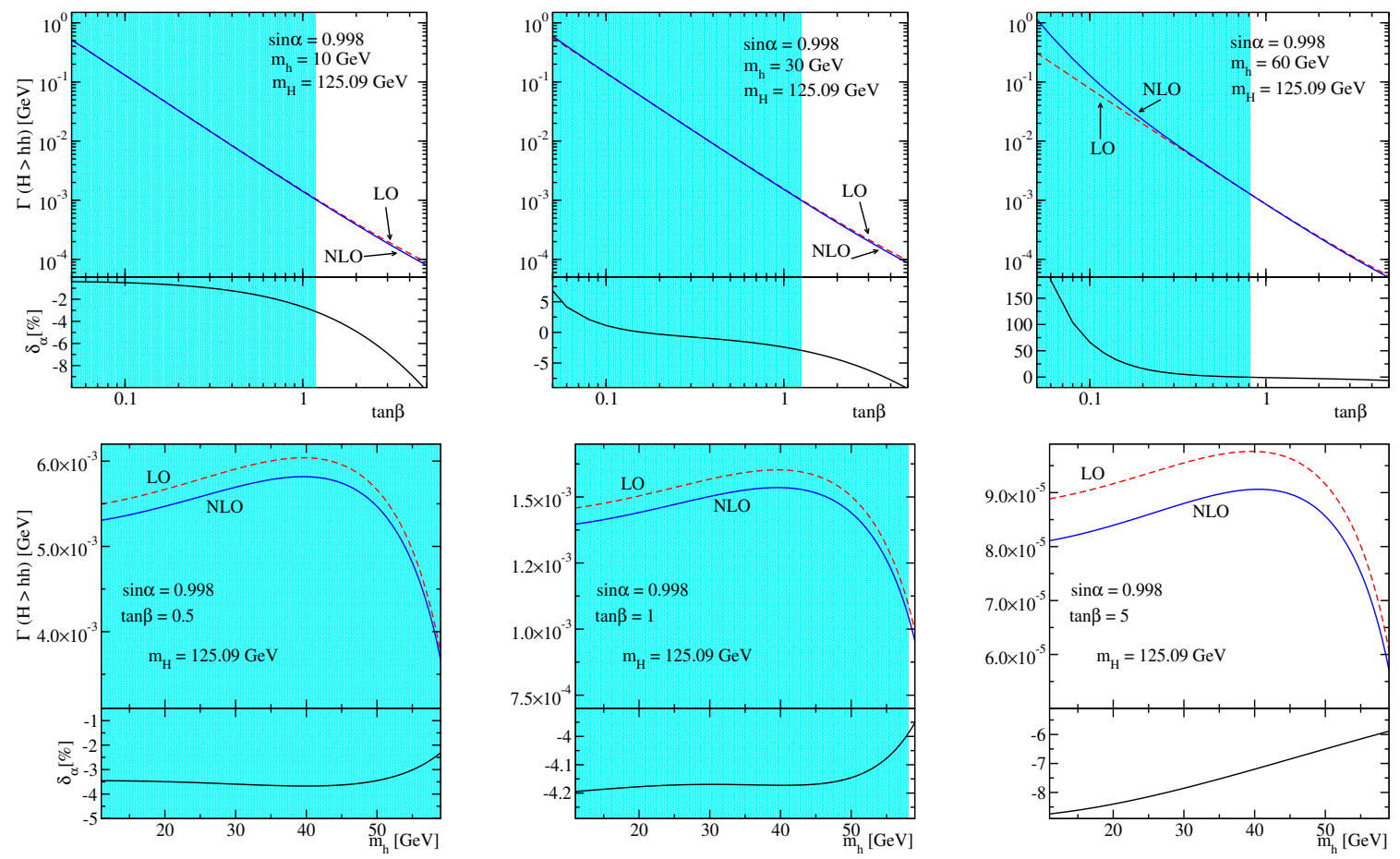

Figure 8. Heavy-to-light Higgs boson width $\Gamma_{H \rightarrow h h}$ in the low-mass region. The results are shown at LO (dashed, red) and NLO (full, blue) as a function of the relevant parameters of the model. The mixing angle is fixed to $\sin \alpha=0.998$ in all cases. The lower subpanels show the relative EW one-loop correction in the $\alpha_{\mathrm{em}}$-parametrization (4.7). Renormalization is performed in the improved on-shell scheme. The shaded areas in the low $\tan \beta$ and $m_{h}$ ranges are ruled out by the LHC Higgs signal strength measurements.

The scale dependence of the NLO results in this low-mass region is analysed in figure 10. The $\Gamma_{H \rightarrow h h}^{\mathrm{NLO}}$ predictions obtained in the improved OS and the minimal field schemes schemes agree very well in the ballpark of the geometric average mass $\mu_{R}^{2}=p_{*}^{2}=\left(m_{h}^{2}+m_{H}^{2}\right) / 2$, and the latter barely varies with the scale. The very stable slope even in the $\tan \beta<1$ region, which is in contrast to the strong scale dependence in the high-mass region, is explained by the much lower scales $\mu_{R}^{2} \simeq p_{*}^{2}$ involved in this case, for which the finite Higgs-mediated contributions to the Higgs field two-point functions are much smaller.

In figure 11 we recast the above analysis in terms of the heavy Higgs branching ratios. We track down their behavior as a function $\tan \beta$ for exemplary light Higgs masses and fiducial mixing $\sin \alpha=0.998$. From values of $\tan \beta \lesssim 1$ onwards, the obtained decay pattern approaches that of a purely SM-like Higgs boson. The dominant mode is $b \bar{b}$, while the di-Higgs final state is hampered due to the tiny tree-level coupling $\lambda_{H h h} \sim c_{\alpha}$. In this case, the $H \rightarrow h h$ mode carries not more than a few percent of the total budget - on equal footing with the loop-induced decay $H \rightarrow g g$. If we instead move towards lower $\tan \beta$ values, the $\cot \beta$-enhanced terms overcome in part the $\sin \alpha \rightarrow 1$ suppression and promote $H \rightarrow h h$ again to a chief role. 

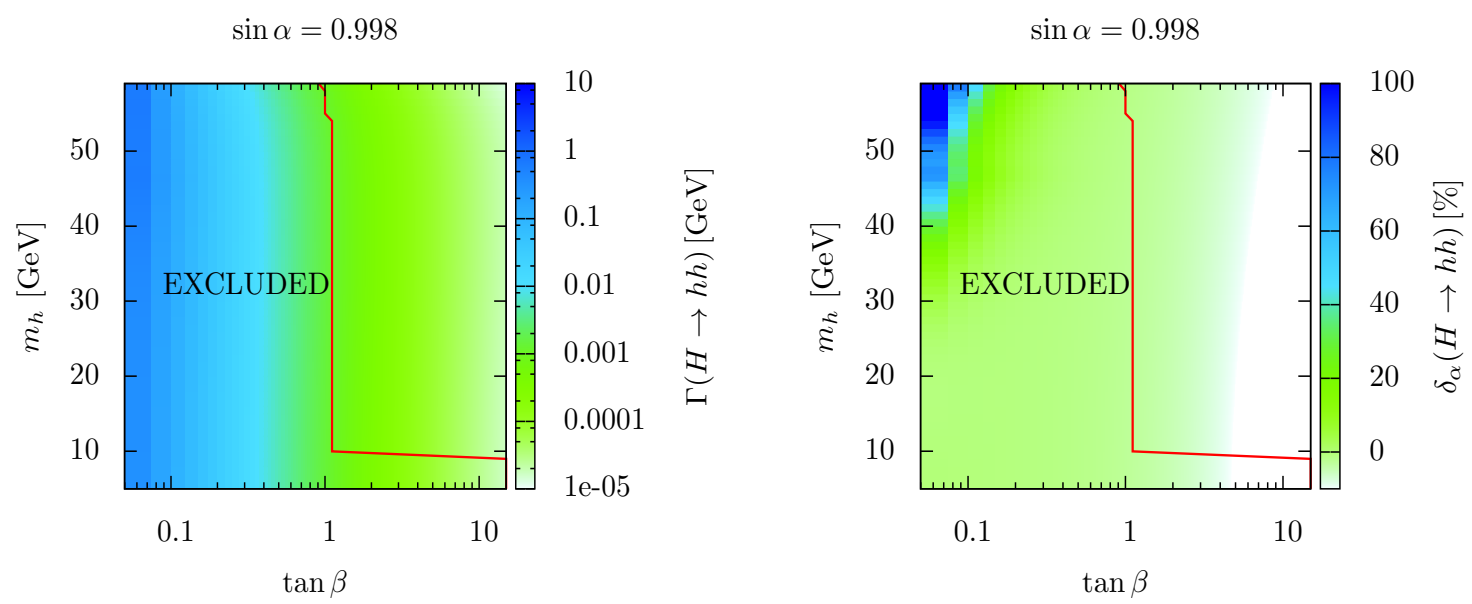

Figure 9. Loop-corrected partial $H \rightarrow h h$ width (left panel) and relative one-loop EW corrections

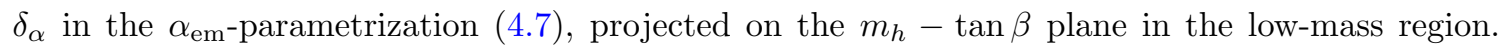
The mixing angle is fixed to the fiducial choice $\sin \alpha=0.998$. Renormalization is performed in the improved on-shell scheme. The regions on the left and below the red contour are excluded by constraints. The strong exclusion in the low mass region follows from direct LEP searches [134].
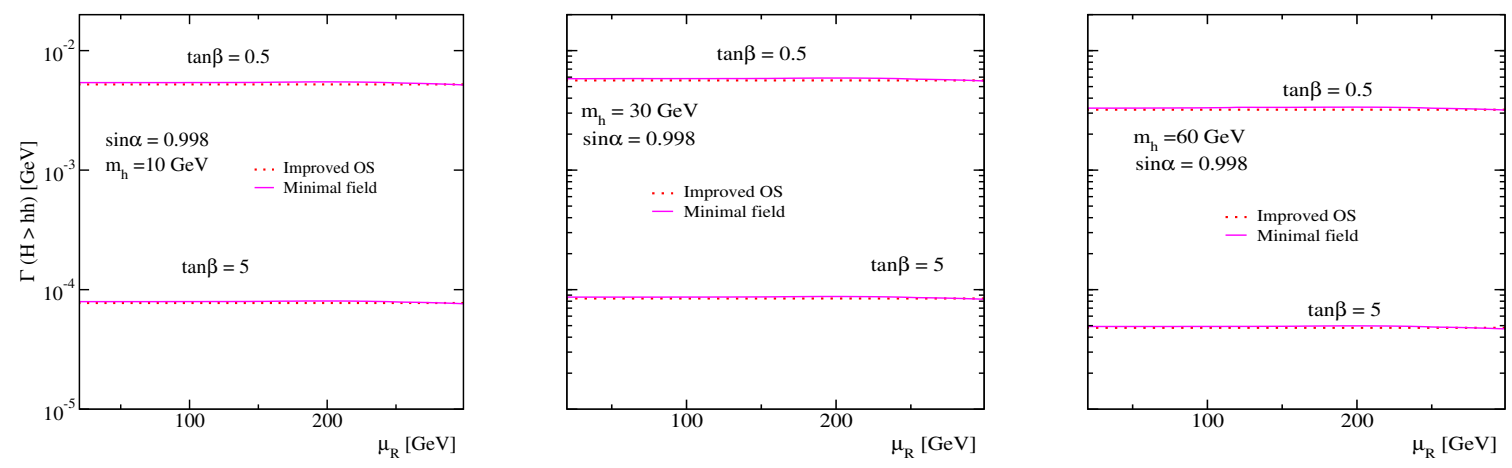

Figure 10. NLO decay width $\Gamma_{H \rightarrow h h}^{\mathrm{NLO}}$ as a function of the renormalization scale in the lowmass region, for exemplary heavy Higgs masses and $\tan \beta$ values. The mixing angle is fixed to $\sin \alpha=0.998$. The scale-dependent predictions for the minimal field scheme are represented by the solid (magenta) lines. The scale-independent reference value (dotted, red lines) we obtain in the improved OS scheme. Parameter space constraints are not shown.

\subsection{Maximal branching ratios}

So far we have discussed the general behavior of the NLO EW corrections to the heavy-tolight Higgs decay width along the relevant parameter space directions $\sin \alpha, \tan \beta, m_{h / H}$. Before closing, we focus on the series of benchmarks with maximal tree-level heavy-tolight Higgs branching ratio proposed in [90]. These are defined as a function of the heavy Higgs through the parameter choices quoted in tables 8, for the high and the low mass regions respectively. In these regimes, the decays of the heavy Higgs state provide a particularly interesting phenomenological ground for studying finite width effects and lineshape modifications in the production of a heavy scalar resonance, cf. e.g. [135-140]. ${ }^{12}$

\footnotetext{
${ }^{12}$ See also e.g. ref. [53] in the context of Higgs pair production.
} 


\begin{tabular}{|c|c|c|c|c|c|}
\hline$m_{H}[\mathrm{GeV}]$ & $\Gamma_{\alpha}^{\mathrm{LO}}(H \rightarrow h h)[\mathrm{GeV}]$ & & $\Gamma_{\alpha}^{\mathrm{NLO}}(H \rightarrow h h)[\mathrm{GeV}]$ & $\delta_{\alpha}[\%]$ & $\delta_{G_{F}}[\%]$ \\
\hline \multicolumn{6}{|c|}{$\tan \beta=0.5, \quad \sin \alpha=0.998$} \\
\hline 10 & $5.496 \times 10^{-3}$ & $\begin{array}{l}\text { OS } \\
\text { Improved OS } \\
\text { Minimal field }\end{array}$ & $\begin{array}{l}5.416 \times 10^{-3} \\
5.415 \times 10^{-3} \\
5.414 \times 10^{-3}\end{array}$ & $\begin{array}{l}-1.458 \\
-1.480 \\
-1.489\end{array}$ & $\begin{array}{l}-1.456 \\
-1.479 \\
-1.488\end{array}$ \\
\hline 30 & $5.920 \times 10^{-3}$ & $\begin{array}{l}\text { OS } \\
\text { Improved OS } \\
\text { Minimal field }\end{array}$ & $\begin{array}{l}5.841 \times 10^{-3} \\
5.844 \times 10^{-3} \\
5.844 \times 10^{-3}\end{array}$ & $\begin{array}{l}-1.345 \\
-1.289 \\
-1.281 \\
\end{array}$ & $\begin{array}{l}-1.344 \\
-1.288 \\
-1.280 \\
\end{array}$ \\
\hline 60 & $3.267 \times 10^{-3}$ & $\begin{array}{l}\text { OS } \\
\text { Improved OS } \\
\text { Minimal field }\end{array}$ & $\begin{array}{l}3.333 \times 10^{-3} \\
3.323 \times 10^{-3} \\
3.331 \times 10^{-3}\end{array}$ & $\begin{array}{l}2.019 \\
1.733 \\
1.952 \\
\end{array}$ & $\begin{array}{l}2.017 \\
1.731 \\
1.949 \\
\end{array}$ \\
\hline \multicolumn{6}{|c|}{$\tan \beta=5, \quad \sin \alpha=0.998$} \\
\hline 10 & $8.881 \times 10^{-5}$ & $\begin{array}{l}\text { OS } \\
\text { Improved OS } \\
\text { Minimal field }\end{array}$ & $\begin{array}{l}7.966 \times 10^{-5} \\
7.967 \times 10^{-5} \\
7.958 \times 10^{-5}\end{array}$ & $\begin{array}{l}-10.310 \\
-10.296 \\
-10.394 \\
\end{array}$ & $\begin{array}{l}-10.216 \\
-10.202 \\
-10.300 \\
\end{array}$ \\
\hline 30 & $9.567 \times 10^{-5}$ & $\begin{array}{l}\text { OS } \\
\text { Improved OS } \\
\text { Minimal field }\end{array}$ & $\begin{array}{l}8.686 \times 10^{-5} \\
8.687 \times 10^{-5} \\
8.680 \times 10^{-5}\end{array}$ & $\begin{array}{l}-9.212 \\
-9.201 \\
-9.279 \\
\end{array}$ & $\begin{array}{l}-9.128 \\
-9.118 \\
-9.195 \\
\end{array}$ \\
\hline 60 & $5.279 \times 10^{-5}$ & $\begin{array}{l}\text { OS } \\
\text { Improved OS } \\
\text { Minimal field }\end{array}$ & $\begin{array}{l}4.931 \times 10^{-5} \\
4.932 \times 10^{-5} \\
4.929 \times 10^{-5}\end{array}$ & $\begin{array}{l}-6.589 \\
-6.584 \\
-6.627\end{array}$ & $\begin{array}{l}-6.529 \\
-6.525 \\
-6.567\end{array}$ \\
\hline
\end{tabular}

Table 7. Heavy-to-light Higgs decay width $\Gamma_{H \rightarrow h h}$ at LO and NLO EW accuracy for representative parameter choices and renormalization schemes in the low-mass region. The total decay widths are obtained in the $\alpha_{\mathrm{em}}$-parametrization, as defined in eqs. (4.7), while the relative one-loop EW effects are quantified in both the $\alpha$-parametrization and the $G_{F}$-parametrization, cf. eq. (5.4). For the (scale-dependent) minimal field scheme, the renormalization scale is fixed to the geometrical average mass $\mu_{R}^{2}=p_{*}^{2}=\left(m_{h}^{2}+m_{H}^{2}\right) / 2$. The input value for $\tan \beta$ is linked to the singlet vev through $v_{s}=\left(\sqrt{2} G_{F}\right)^{-1 / 2} \tan \beta$.
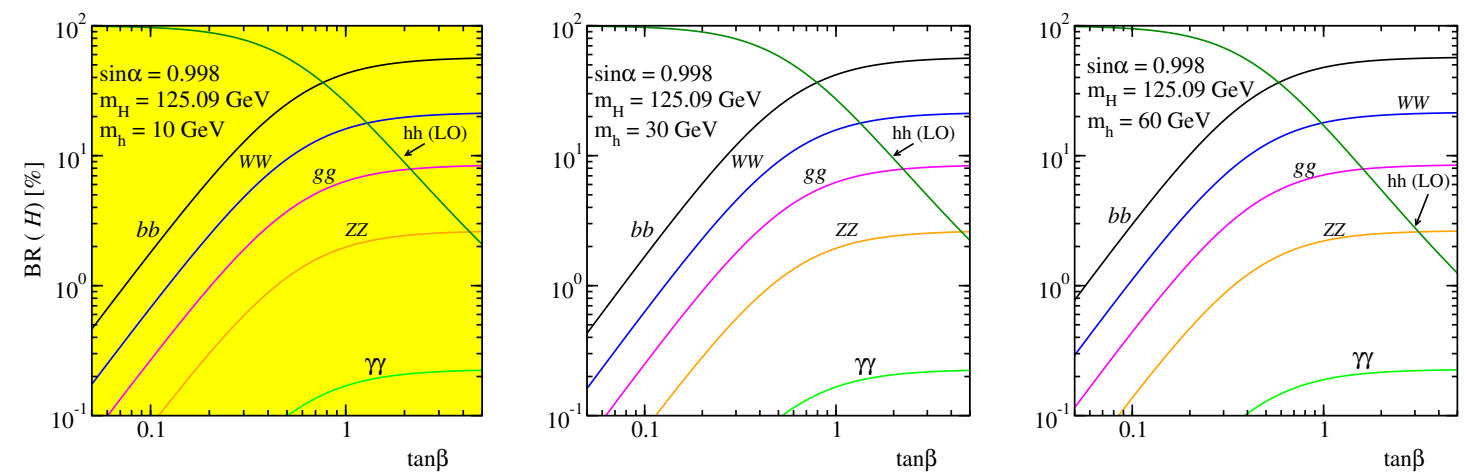

Figure 11. Heavy Higgs branching ratios (in \%) as a function of $\tan \beta$ in the low-mass region. The results are shown for representative light Higgs mass values, with fiducial mixing angle $\sin \alpha=0.998$. The whole parameter space region shown in the left panel is excluded by perturbative unitarity. 


\begin{tabular}{|lcccc|ccccc|}
\hline \multicolumn{5}{|c|}{ high mass region } & \multicolumn{5}{c|}{ low mass region } \\
\hline & $m_{H}[\mathrm{GeV}]$ & $|\sin \alpha|_{\max }$ & $B R_{\max }^{H \rightarrow h h}$ & $\tan \beta$ & & $m_{h}[\mathrm{GeV}]$ & $|\sin \alpha|_{\min }$ & $B R_{\max }^{H \rightarrow h h}$ & $\tan \beta$ \\
\hline BHM1 & 300 & 0.31 & 0.34 & 3.71 & BLM1 & 60 & 0.9997 & 0.26 & 0.29 \\
BHM2 & 400 & 0.27 & 0.32 & 1.72 & BLM2 & 50 & 0.9998 & 0.26 & 0.31 \\
BHM3 & 500 & 0.24 & 0.27 & 2.17 & BLM3 & 40 & 0.9998 & 0.26 & 0.32 \\
BHM4 & 600 & 0.23 & 0.25 & 2.70 & BLM4 & 30 & 0.9998 & 0.26 & 0.32 \\
BHM5 & 700 & 0.21 & 0.24 & 3.23 & BLM5 & 20 & 0.9998 & 0.26 & 0.31 \\
BHM6 & 800 & 0.21 & 0.23 & 4.00 & BLM6 & 10 & 0.9998 & 0.26 & 0.30 \\
\hline
\end{tabular}

Table 8. Maximal branching ratios for the heavy-to-light Higgs decay mode $H \rightarrow h h$ in the high-mass (left) and low-mass regions (right) as proposed in refs. [90, 91]; the results quoted here are obtained in the setup of the mentioned references, evaluating $\Gamma(H \rightarrow h h)$ at LO in the $G_{F^{-}}$ parametrization. Note that the maximal branching ratios are determined for a maximal mixing, to ensure a large production rate. In this case, the lower limit of $\tan \beta$ is mainly determined by the requirement of perturbativity for $\lambda_{2}$, cf. the extensive discussion in [20]. The same strategy was followed for the low-mass region, where again for fixed $\sin \alpha$ values the minimal value of $\tan \beta$ is determined. Here, the lower limit on $\tan \beta$ stems from the signal strength fit.

\begin{tabular}{|l|llll|llllll|r|}
\hline & $\Gamma_{H \rightarrow h h}^{\mathrm{LO}}$ & $\Gamma_{H \rightarrow h h}^{\mathrm{NLO}}$ & $\delta_{\alpha}[\%]$ & $\delta_{G_{F}}[\%]$ & $b \bar{b}$ & $t \bar{t}$ & $\mathrm{WW}$ & $\mathrm{ZZ}$ & $g g$ & $h h$ & $\Gamma_{H}$ \\
\hline BHM1 & 0.399 & 0.413 & 3.411 & 3.291 & 0.04 & $<0.01$ & 46.35 & 20.56 & 0.04 & 33.02 & 1.210 \\
BHM2 & 0.963 & 1.026 & 6.485 & 6.272 & 0.01 & 10.19 & 40.07 & 18.52 & 0.06 & 31.15 & 3.092 \\
BHM3 & 1.383 & 1.463 & 5.803 & 5.604 & 0.01 & 14.19 & 40.36 & 19.29 & 0.04 & 26.09 & 5.299 \\
BHM4 & 2.067 & 2.161 & 4.520 & 4.361 & 0.01 & 12.82 & 42.35 & 20.64 & 0.03 & 24.11 & 8.574 \\
BHM5 & 2.637 & 2.717 & 3.027 & 2.918 & $<0.01$ & 10.61 & 44.37 & 21.91 & 0.02 & 23.11 & 11.413 \\
BHM6 & 3.798 & 3.867 & 1.826 & 1.759 & $<0.01$ & 8.57 & 46.29 & 23.07 & 0.02 & 22.07 & 17.204 \\
\hline
\end{tabular}

Table 9. Heavy-to-light Higgs decay width $\Gamma_{H \rightarrow h h}$ at LO and NLO EW accuracy for the maximal branching fraction scenarios in the high-mass region given in table 8 . The relative one-loop EW effects are quantified in both the $\alpha_{\mathrm{em}}$-parametrization and the $G_{F}$-parametrization, as defined in eqs. (4.7)-(5.4). Renormalization is performed in the improved on-shell scheme. In the right columns we document the branching ratios (in \%) for the leading Higgs decay channels and the total Higgs width. Like in figures 6 and 11, all partial decay widths to SM fields are evaluated by rescaling the SM predictions [44], while for $H \rightarrow h h$ we use the LO result evaluated in the

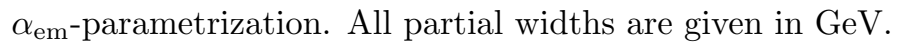

Numerical predictions for $\Gamma_{H \rightarrow h h}^{\mathrm{LO}}$ and $\Gamma_{H \rightarrow h h}^{\mathrm{NLO}}$, together with the relative one-loop correction in the two parametrizations $\delta_{\alpha_{\mathrm{em}}}$ and $\delta_{\mathrm{GF}}(5.4)$ are provided in tables 9 and 10, for the high and low mass regions respectively. Complementarily, we list down the corresponding branching fractions for the additional decay modes (barring those channels below $0.01 \%$ ). Let us recall that for the latter we use the rescaled partial widths from [44], while for $H \rightarrow h h$ we quote the LO result in the $\alpha_{\mathrm{em}}$-parametrization, in line with figures 6 and 11. 


\begin{tabular}{|l|llll|cccccc|l|}
\hline & $\Gamma_{H \rightarrow h h}^{\mathrm{LO}}$ & $\Gamma_{H \rightarrow h h}^{\mathrm{NLO}}$ & $\delta_{\alpha}[\%]$ & $\delta_{G_{F}}[\%]$ & $b \bar{b}$ & $\gamma \gamma$ & $\mathrm{WW}$ & $\mathrm{ZZ}$ & $g g$ & $h h$ & $\Gamma_{H}$ \\
\hline BLM1 & 1.426 & 1.536 & 7.765 & 7.763 & 42.65 & 0.17 & 16.04 & 1.97 & 6.34 & 25.90 & 5.506 \\
BLM2 & 1.439 & 1.472 & 2.305 & 2.304 & 42.55 & 0.17 & 16.00 & 1.97 & 6.33 & 26.07 & 5.520 \\
BLM3 & 1.423 & 1.432 & 0.586 & 0.586 & 42.67 & 0.17 & 16.05 & 1.97 & 6.35 & 25.86 & 5.504 \\
BLM4 & 1.419 & 1.415 & -0.272 & -0.272 & 42.71 & 0.17 & 16.06 & 1.97 & 6.35 & 25.80 & 5.500 \\
BLM5 & 1.431 & 1.425 & -0.445 & -0.445 & 42.61 & 0.17 & 16.02 & 1.97 & 6.34 & 25.96 & 5.512 \\
BLM6 & 1.427 & 1.421 & -0.438 & -0.438 & 42.64 & 0.17 & 16.04 & 1.97 & 6.34 & 25.91 & 5.508 \\
\hline
\end{tabular}

Table 10. As in table 9 for the low-mass region. Notice that in this case all partial widths are given in $\mathrm{MeV}$.

\section{Summary}

Heavy-to-light Higgs decays $H \rightarrow h h$ are of undisputed relevance in the phenomenological characterization of extended Higgs sectors. When kinematically accessible, these may contribute to, and in some scenarios even dominate, the heavy Higgs lineshape, while at the same time they significantly modify its decay pattern with respect to the SM picture. On the other hand, both the tree-level and the leading one-loop contributions to this process are governed by the scalar self-interactions, which makes this decay a unique handle on the architecture of the scalar potential.

While electroweak corrections to the Higgs self-couplings have been the subject of dedicated analyses in the 2HDM [141-143], the NMSSM [144] or the Inert Doublet model [145], a corresponding study for the singlet extension was lacking. Extending upon previous work [19], we have presented herewith a detailed analysis of the heavy-to-light Higgs decays at NLO electroweak accuracy. To renormalize the singlet-extended Higgs sector we have proposed four renormalization schemes: i) a minimal field setup; ii) a traditional onshell prescription; iii) a mixed $\overline{\mathrm{MS}}$ /on-shell scheme; and iv) an improved on-shell scheme. Using the general non-linear gauge-fixing of SLOops, we have discussed in detail the gauge independence of the different renormalization setups. We have found that, while the minimal field and on-shell approaches still lead to a residual dependence on the non-linear gauge fixing parameters, the mixed $\overline{\mathrm{MS}} / \mathrm{OS}$ and improved OS schemes render gauge independent one-loop predictions for physical observables. Furthermore, the improved OS scheme is numerically stable in all regions of the parameter space. We therefore advocate for the use of this scheme to investigate the phenomenology of singlet extensions of the SM at higher orders.

We have applied the above schemes to compute the corresponding heavy-to-light Higgs decay widths $\Gamma_{H \rightarrow h h}$ including one-loop electroweak corrections. We have performed a comprehensive phenomenological analysis, with a separate study of two possible realizations of the model: a high-mass and a low-mass region, in which the additional scalar field corresponds to a heavy (resp. a light) companion of the SM-like Higgs boson.

The phenomenological implications of our study can be summarized as follows:

- The heavy-to-light Higgs decay width at LO is governed by two competing mechanisms: i) the Higgs self-coupling strength $\lambda_{H h h}$; ii) the one-to-two body decay 
kinematics. We pinpoint a strongly varying width with the relevant model parameters. Overall, $\Gamma_{H \rightarrow h h}$ may attain up to $\mathcal{O}(1) \mathrm{GeV}$ for heavy Higgs masses above $m_{H} \sim 500 \mathrm{GeV}$.

- Aside from the $\tan \beta<1$ region, the relative one-loop effects are mild and show tempered variations over the parameter space. In the high-mass region, electroweak corrections are positive, loosely variable, and stagnate in the ballpark of few percent. In the low-mass region, mainly for $\tan \beta>1$ and small $m_{h}$ values, these may be pulled down to $\delta_{\alpha} \sim-10 \%$.

- For certain parameter choices, the $H \rightarrow h h$ decay becomes effectively loop-induced: i) along the tree-level nodes where the LO contribution vanishes; ii) at low $\tan \beta$, where the $\cot \beta$-enhancements lead to increased scalar self-couplings, and thereby to large Higgs-mediated loop graphs. In practice, though, these sizable quantum effects are precluded once the constraints on the model are taken into account.

- Let alone extreme parameter space corners, the NLO predictions are robust under changes of renormalization schemes and renormalization scale choices, as indicative of a small theoretical uncertainty.

Having constructed a complete renormalization scheme for the Higgs sector, the path ahead is clear for further analyses on the topic. On the one hand, it will be interesting to further explore the role of the quantum effects on the Higgs self-couplings themselves, and whether these may have relevant implications e.g. for collider searches or in electroweak baryogenesis. On the other hand, the complete renormalization of the Higgs sector paves the way towards characterizing the singlet model phenomenology at one-loop electroweak accuracy, including all Higgs production modes and decay channels, and exploiting the rich possibilities of off-shell effects. Work in this direction is underway [131].

\section{Acknowledgments}

We are grateful to Fawzi Boudjema, Sven Heinemeyer, Dominik Stöckinger and Hyejung Stöckinger-Kim for enlightening discussions. DLV acknowledges the support of the F.R.S.-FNRS "Fonds de la Recherche Scientifique" (Belgium). The work of GC is supported by the Theory-LHC-France initiative of CNRS/IN2P3.

\section{A Analytical details}

\section{A.1 Feynman rules}

For a sake of completeness, we collect the relevant Feynman rules for the three-point and four-point scalar field self-interactions in the singlet model. The complete set of Feynman rules has been arranged in the form of a FEYNARTs model file [127], which we have obtained via two independent implementations using FeYnRules [129] and SLOOPS [15-18]. Here we give the results in the 't Hooft-Feynman gauge. The shorthand notation $s_{\theta} \equiv \sin (\theta), c_{\theta} \equiv \cos (\theta) t_{\theta} \equiv \tan (\theta)$ is employed throughout. 
- Trilinear self-couplings at tree-level:

$$
\begin{aligned}
\lambda_{h h h} & =-3 i\left(2 c_{\alpha}^{3} \lambda_{1} v+c_{\alpha} s_{\alpha}^{2} \lambda_{3} v-c_{\alpha}^{2} s_{\alpha} \lambda_{3} v_{s}-2 s_{\alpha}^{3} \lambda_{2} v_{s}\right)=-\frac{3 i m_{h}^{2}}{v}\left(c_{\alpha}^{3}-s_{\alpha}^{3} t_{\beta}^{-1}\right) \\
\lambda_{H h h} & =-i\left(6 c_{\alpha}^{2} s_{\alpha} \lambda_{1} v-2 c_{\alpha}^{2} s_{\alpha} \lambda_{3} v+s_{\alpha}^{3} \lambda_{3} v+c_{\alpha}^{3} \lambda_{3} v_{s}-2 c_{\alpha} s_{\alpha}^{2} \lambda_{3} v_{s}+6 c_{\alpha} s_{\alpha}^{2} \lambda_{2} v_{s}\right) \\
& =-\frac{i s_{2 \alpha}}{v}\left[m_{h}^{2}+\frac{m_{H}^{2}}{2}\right]\left(c_{\alpha}+s_{\alpha} t_{\beta}^{-1}\right) \\
\lambda_{H H h} & =-i\left(6 c_{\alpha} s_{\alpha}^{2} \lambda_{1} v-2 c_{\alpha} s_{\alpha}^{2} \lambda_{3} v+c_{\alpha}^{3} \lambda_{3} v-s_{\alpha}^{3} \lambda_{3} v_{s}+2 c_{\alpha}^{2} s_{\alpha} \lambda_{3} v_{s}-6 c_{\alpha}^{2} s_{\alpha} \lambda_{2} v_{s}\right) \\
& =\frac{i s_{2 \alpha}}{v}\left[\frac{m_{h}^{2}}{2}+m_{H}^{2}\right]\left(-s_{\alpha}+c_{\alpha} t_{\beta}^{-1}\right) \\
\lambda_{H H H} & =-\frac{3 i m_{H}^{2}}{v}\left(c_{\alpha}^{3} t_{\beta}^{-1}+s_{\alpha}^{3}\right) .
\end{aligned}
$$

- Higgs-Goldstone boson three-point couplings:

$$
\lambda_{h \mathrm{G}^{0} \mathrm{G}^{0}}=\lambda_{H \mathrm{G}^{+} \mathrm{G}^{-}}=(-i) m_{h}^{2} c_{\alpha} / v ; \quad \lambda_{H \mathrm{G}^{0} \mathrm{G}^{0}}=\lambda_{H \mathrm{G}^{+} \mathrm{G}^{-}}=(-i) m_{H}^{2} s_{\alpha} / v .
$$

- Higgs quartic couplings:

$$
\begin{aligned}
\lambda_{h h h h} & =-\frac{3 i}{v^{2}}\left[m_{h}^{2} c_{\alpha}^{6}+m_{H}^{2} c_{\alpha}^{4} s_{\alpha}^{2}-2\left(m_{h}^{2}-m_{H}^{2}\right) c_{\alpha}^{3} t_{\beta}^{-1} s_{\alpha}^{3}+m_{H}^{2} c_{\alpha}^{2} s_{\alpha}^{4} t_{\beta}^{-2}+m_{h}^{2} t_{\beta}^{-2} s_{\alpha}^{6}\right] \\
\lambda_{H h h h} & =\frac{3 i}{8 v^{2} s_{\beta}^{2}} s_{2 \alpha} s_{\alpha+\beta}\left[\left(3 m_{h}^{2}+m_{H}^{2}\right) s_{\alpha-\beta}+\left(m_{H}^{2}-m_{h}^{2}\right) s_{3 \alpha+\beta}\right] \\
\lambda_{H H h h} & =-\frac{i}{16 v^{2} s_{\beta}^{2}} s_{2 \alpha}\left[6\left(m_{h}^{2}+m_{H}^{2}\right) s_{2 \alpha}-\left(m_{h}^{2}-m_{H}^{2}\right)\left(s_{2 \beta}+3 s_{4 \alpha+2 \beta}\right)\right] \\
\lambda_{H H H h} & =\frac{i}{8 v^{2} s_{\beta}^{2}} s_{2 \alpha} c_{\alpha+\beta}\left[\left(m_{h}^{2}+3 m_{H}^{2}\right) c_{\alpha-\beta}+\left(m_{H}^{2}-m_{h}^{2}\right) c_{3 \alpha+\beta}\right] \\
\lambda_{H H H H} & =-\frac{3 i}{v^{2}}\left[m_{H}^{2} c_{\alpha}^{6} t_{\beta}^{-2}+m_{h}^{2} c_{\alpha}^{4} s_{\alpha}^{2} t_{\beta}^{-2}-2\left(m_{h}^{2}-m_{H}^{2}\right) c_{\alpha}^{3} t_{\beta}^{-1} s_{\alpha}^{3}+m_{h}^{2} c_{\alpha}^{2} s_{\alpha}^{4}+m_{H}^{2} s_{\alpha}^{6}\right]
\end{aligned}
$$

- Higgs-Goldstone four-point couplings:

$$
\begin{aligned}
\lambda_{h h \mathrm{G}^{0} \mathrm{G}^{0}} & =\lambda_{h h \mathrm{G}^{+} \mathrm{G}^{-}}=\frac{-i}{v^{2}} c_{\alpha}\left[m_{h}^{2} c_{\alpha}^{3}+m_{H}^{2} s_{\alpha}^{2} c_{\alpha}+\left(m_{H}^{2}-m_{h}^{2}\right) s_{\alpha}^{3} t_{\beta}^{-1}\right] \\
\lambda_{H H \mathrm{G}^{0} \mathrm{G}^{0}} & =\lambda_{H H \mathrm{G}^{+} \mathrm{G}^{-}}=\frac{-i}{v^{2}} s_{\alpha}\left[m_{h}^{2} c_{\alpha}^{2} s_{\alpha}+m_{H}^{2} s_{\alpha}^{3}+\left(m_{H}^{2}-m_{h}^{2}\right) c_{\alpha}^{3} t_{\beta}^{-1}\right]
\end{aligned}
$$

\section{A.2 Trilinear Higgs counterterm}

The coefficients entering the trilinear Higgs coupling counterterm $\delta \lambda_{H \rightarrow h h}$ in eq. (4.6) yield

$$
\begin{aligned}
& c_{1}^{H h h}=\frac{s_{2 \alpha} s_{\alpha+\beta}}{v s_{\beta}} ; \quad c_{2}^{H h h}=\frac{s_{2 \alpha} s_{\alpha+\beta}}{2 v s_{\beta}} ; \quad c_{3}^{H h h}=-\frac{s_{\beta+3 \alpha}-5 s_{\beta-\alpha}}{4 v s_{\beta}} \\
& c_{4}^{H h h}=\frac{3 s_{2 \alpha}\left(c_{\alpha}^{2}-s_{\alpha}^{2} t_{\beta}^{-2}\right)}{2 v^{2}} \quad c_{5}^{H h h}=\frac{3 c_{\alpha}^{2} s_{\alpha}^{2}}{s_{\beta}^{2} v^{2}} ; \quad c_{6}^{H h h}=-\frac{s_{2 \alpha}}{v}\left[m_{h}^{2}+\frac{m_{H}^{2}}{2}\right] c_{\alpha} .
\end{aligned}
$$


Open Access. This article is distributed under the terms of the Creative Commons Attribution License (CC-BY 4.0), which permits any use, distribution and reproduction in any medium, provided the original author(s) and source are credited.

\section{References}

[1] ATLAS collaboration, Observation of a new particle in the search for the Standard Model Higgs boson with the ATLAS detector at the LHC, Phys. Lett. B 716 (2012) 1 [arXiv: 1207.7214] [INSPIRE].

[2] CMS collaboration, Observation of a new boson at a mass of $125 \mathrm{GeV}$ with the CMS experiment at the LHC, Phys. Lett. B 716 (2012) 30 [arXiv:1207.7235] [INSPIRE].

[3] P.W. Higgs, Broken Symmetries and the Masses of Gauge Bosons, Phys. Rev. Lett. 13 (1964) 508 [INSPIRE].

[4] P.W. Higgs, Broken symmetries, massless particles and gauge fields, Phys. Lett. 12 (1964) 132 [INSPIRE].

[5] F. Englert and R. Brout, Broken Symmetry and the Mass of Gauge Vector Mesons, Phys. Rev. Lett. 13 (1964) 321 [INSPIRE].

[6] G.S. Guralnik, C.R. Hagen and T.W.B. Kibble, Global Conservation Laws and Massless Particles, Phys. Rev. Lett. 13 (1964) 585 [InSPIRE].

[7] M. Spira and P.M. Zerwas, Electroweak symmetry breaking and Higgs physics, Lect. Notes Phys. 512 (1998) 161 [hep-ph/9803257] [INSPIRE].

[8] A. Djouadi, The anatomy of electro-weak symmetry breaking. I: The Higgs boson in the standard model, Phys. Rept. 457 (2008) 1 [hep-ph/0503172] [INSPIRE].

[9] T. Plehn, Lectures on LHC Physics, Lect. Notes Phys. 844 (2012) 1 [arXiv:0910.4182] [INSPIRE].

[10] C. Englert et al., Precision Measurements of Higgs Couplings: Implications for New Physics Scales, J. Phys. G 41 (2014) 113001 [arXiv: 1403.7191] [InSPIRE].

[11] V. Silveira and A. Zee, Scalar phantoms, Phys. Lett. B 161 (1985) 136 [inSPIRE].

[12] R. Schabinger and J.D. Wells, A minimal spontaneously broken hidden sector and its impact on Higgs boson physics at the large hadron collider, Phys. Rev. D 72 (2005) 093007 [hep-ph/0509209] [INSPIRE].

[13] B. Patt and F. Wilczek, Higgs-field portal into hidden sectors, hep-ph/0605188 [INSPIRE].

[14] M. Bowen, Y. Cui and J.D. Wells, Narrow trans-TeV Higgs bosons and $H \rightarrow$ hh decays: Two LHC search paths for a hidden sector Higgs boson, JHEP 03 (2007) 036 [hep-ph/0701035] [INSPIRE].

[15] F. Boudjema, A. Semenov and D. Temes, Self-annihilation of the neutralino dark matter into two photons or a $Z$ and a photon in the MSSM, Phys. Rev. D 72 (2005) 055024 [hep-ph/0507127] [INSPIRE].

[16] N. Baro, F. Boudjema and A. Semenov, Full one-loop corrections to the relic density in the MSSM: A few examples, Phys. Lett. B 660 (2008) 550 [arXiv:0710.1821] [INSPIRE].

[17] N. Baro, F. Boudjema and A. Semenov, Automatised full one-loop renormalisation of the MSSM. I. The Higgs sector, the issue of $\tan \beta$ and gauge invariance, Phys. Rev. D 78 (2008) 115003 [arXiv:0807.4668] [INSPIRE]. 
[18] N. Baro and F. Boudjema, Automatised full one-loop renormalisation of the MSSM II: The chargino-neutralino sector, the sfermion sector and some applications, Phys. Rev. D 80 (2009) 076010 [arXiv:0906.1665] [INSPIRE].

[19] D. López-Val and T. Robens, $\Delta r$ and the $W$-boson mass in the singlet extension of the standard model, Phys. Rev. D 90 (2014) 114018 [arXiv:1406.1043] [INSPIRE].

[20] G.M. Pruna and T. Robens, Higgs singlet extension parameter space in the light of the LHC discovery, Phys. Rev. D 88 (2013) 115012 [arXiv:1303.1150] [InSPIRE].

[21] T. Robens and T. Stefaniak, Status of the Higgs Singlet Extension of the Standard Model after LHC Run 1, Eur. Phys. J. C 75 (2015) 104 [arXiv: 1501.02234] [inSPIRE].

[22] U. Ellwanger, C. Hugonie and A.M. Teixeira, The Next-to-Minimal Supersymmetric Standard Model, Phys. Rept. 496 (2010) 1 [arXiv:0910.1785] [InSPIRE].

[23] J. Hetzel and B. Stech, Low-energy phenomenology of trinification: an effective left-right-symmetric model, Phys. Rev. D 91 (2015) 055026 [arXiv: 1502.00919] [INSPIRE].

[24] L. Basso, S. Moretti and G.M. Pruna, A Renormalisation Group Equation Study of the Scalar Sector of the Minimal B-L Extension of the Standard Model, Phys. Rev. D 82 (2010) 055018 [arXiv: 1004.3039] [INSPIRE].

[25] M.J. Strassler and K.M. Zurek, Echoes of a hidden valley at hadron colliders, Phys. Lett. B 651 (2007) 374 [hep-ph/0604261] [INSPIRE].

[26] D. O'Connell, M.J. Ramsey-Musolf and M.B. Wise, Minimal Extension of the Standard Model Scalar Sector, Phys. Rev. D 75 (2007) 037701 [hep-ph/0611014] [INSPIRE].

[27] O. Bahat-Treidel, Y. Grossman and Y. Rozen, Hiding the Higgs at the LHC, JHEP 05 (2007) 022 [hep-ph/0611162] [InSPIRE].

[28] V. Barger, P. Langacker, M. McCaskey, M.J. Ramsey-Musolf and G. Shaughnessy, LHC Phenomenology of an Extended Standard Model with a Real Scalar Singlet, Phys. Rev. D 77 (2008) 035005 [arXiv:0706 .4311] [INSPIRE].

[29] G. Bhattacharyya, G.C. Branco and S. Nandi, Universal Doublet-Singlet Higgs Couplings and phenomenology at the CERN Large Hadron Collider, Phys. Rev. D 77 (2008) 117701 [arXiv: 0712.2693] [INSPIRE].

[30] M. Gonderinger, Y. Li, H. Patel and M.J. Ramsey-Musolf, Vacuum Stability, Perturbativity and Scalar Singlet Dark Matter, JHEP 01 (2010) 053 [arXiv:0910.3167] [INSPIRE].

[31] S. Dawson and W. Yan, Hiding the Higgs Boson with Multiple Scalars, Phys. Rev. D 79 (2009) 095002 [arXiv:0904.2005] [INSPIRE].

[32] S. Bock, R. Lafaye, T. Plehn, M. Rauch, D. Zerwas and P.M. Zerwas, Measuring Hidden Higgs and Strongly-Interacting Higgs Scenarios, Phys. Lett. B 694 (2011) 44 [arXiv: 1007.2645] [INSPIRE].

[33] P.J. Fox, D. Tucker-Smith and N. Weiner, Higgs friends and counterfeits at hadron colliders, JHEP 06 (2011) 127 [arXiv:1104.5450] [INSPIRE].

[34] C. Englert, T. Plehn, D. Zerwas and P.M. Zerwas, Exploring the Higgs portal, Phys. Lett. B 703 (2011) 298 [arXiv:1106.3097] [InSPIRE].

[35] C. Englert, J. Jaeckel, E. Re and M. Spannowsky, Evasive Higgs Maneuvers at the LHC, Phys. Rev. D 85 (2012) 035008 [arXiv:1111.1719] [InSPIRE].

[36] B. Batell, S. Gori and L.-T. Wang, Exploring the Higgs Portal with $10 \mathrm{fb}^{-1}$ at the LHC, JHEP 06 (2012) 172 [arXiv:1112.5180] [INSPIRE]. 
[37] C. Englert, T. Plehn, M. Rauch, D. Zerwas and P.M. Zerwas, LHC: Standard Higgs and Hidden Higgs, Phys. Lett. B 707 (2012) 512 [arXiv:1112.3007] [InSPIRE].

[38] R.S. Gupta and J.D. Wells, Higgs boson search significance deformations due to mixed-in scalars, Phys. Lett. B 710 (2012) 154 [arXiv:1110.0824] [InSPIRE].

[39] M.J. Dolan, C. Englert and M. Spannowsky, New Physics in LHC Higgs boson pair production, Phys. Rev. D 87 (2013) 055002 [arXiv:1210.8166] [INSPIRE].

[40] D. Bertolini and M. McCullough, The Social Higgs, JHEP 12 (2012) 118 [arXiv: 1207.4209] [INSPIRE].

[41] B. Batell, D. McKeen and M. Pospelov, Singlet Neighbors of the Higgs Boson, JHEP 10 (2012) 104 [arXiv: 1207.6252] [InSPIRE].

[42] F. Bazzocchi and M. Fabbrichesi, A simple inert model solves the little hierarchy problem and provides a dark matter candidate, Eur. Phys. J. C 73 (2013) 2303 [arXiv:1207.0951] [INSPIRE].

[43] D. López-Val, T. Plehn and M. Rauch, Measuring extended Higgs sectors as a consistent free couplings model, JHEP 10 (2013) 134 [arXiv:1308.1979] [INSPIRE].

[44] LhC Higgs Cross section Working Group collaboration, J.R. Andersen et al., Handbook of LHC Higgs Cross sections: 3. Higgs Properties, arXiv:1307.1347 [INSPIRE].

[45] R.S. Chivukula, A. Farzinnia, J. Ren and E.H. Simmons, Constraints on the Scalar Sector of the Renormalizable Coloron Model, Phys. Rev. D 88 (2013) 075020 [arXiv:1307.1064] [INSPIRE].

[46] C. Englert and M. McCullough, Modified Higgs Sectors and NLO Associated Production, JHEP 07 (2013) 168 [arXiv:1303.1526] [InSPIRE].

[47] B. Cooper, N. Konstantinidis, L. Lambourne and D. Wardrope, Boosted $h h \rightarrow b \bar{b} b \bar{b}:$ A new topology in searches for TeV-scale resonances at the LHC, Phys. Rev. D 88 (2013) 114005 [arXiv: 1307.0407] [INSPIRE].

[48] C. Caillol, B. Clerbaux, J.-M. Frère and S. Mollet, Precision versus discovery: A simple benchmark, Eur. Phys. J. Plus 129 (2014) 93 [arXiv:1304.0386] [InSPIRE].

[49] R. Coimbra, M.O.P. Sampaio and R. Santos, ScannerS: Constraining the phase diagram of a complex scalar singlet at the LHC, Eur. Phys. J. C 73 (2013) 2428 [arXiv:1301.2599] [INSPIRE].

[50] A. Eichhorn and M.M. Scherer, Planck scale, Higgs mass and scalar dark matter, Phys. Rev. D 90 (2014) 025023 [arXiv: 1404.5962] [InSPIRE].

[51] V. Martín Lozano, J.M. Moreno and C.B. Park, Resonant Higgs boson pair production in the $h h \rightarrow b \bar{b} W W \rightarrow b \bar{b} \ell^{+} \nu \ell^{-} \bar{\nu}$ decay channel, JHEP 08 (2015) 004 [arXiv:1501.03799] [INSPIRE].

[52] A. Falkowski, C. Gross and O. Lebedev, A second Higgs from the Higgs portal, JHEP 05 (2015) 057 [arXiv: 1502.01361] [InSPIRE].

[53] S. Dawson and I.M. Lewis, NLO corrections to double Higgs boson production in the Higgs singlet model, Phys. Rev. D 92 (2015) 094023 [arXiv: 1508.05397] [INSPIRE].

[54] D. Buttazzo, F. Sala and A. Tesi, Singlet-like Higgs bosons at present and future colliders, JHEP 11 (2015) 158 [arXiv: 1505. 05488] [INSPIRE].

[55] S. Banerjee, M. Mitra and M. Spannowsky, Searching for a Heavy Higgs boson in a Higgs-portal B-L Model, Phys. Rev. D 92 (2015) 055013 [arXiv:1506.06415] [INSPIRE]. 
[56] C. Englert, I. Low and M. Spannowsky, On-shell interference effects in Higgs boson final states, Phys. Rev. D 91 (2015) 074029 [arXiv: 1502.04678] [INSPIRE].

[57] C.-Y. Chen, S. Dawson and I.M. Lewis, Exploring resonant di-Higgs boson production in the Higgs singlet model, Phys. Rev. D 91 (2015) 035015 [arXiv:1410.5488] [INSPIRE].

[58] C. Englert, Y. Soreq and M. Spannowsky, Off-Shell Higgs Coupling Measurements in BSM scenarios, JHEP 05 (2015) 145 [arXiv:1410.5440] [INSPIRE].

[59] ATLAS collaboration, A search for resonant Higgs-pair production in the $b \bar{b} b \bar{b}$ final state in pp collisions at $\sqrt{s}=8 \mathrm{TeV}$, ATLAS-CONF-2014-005 (2014).

[60] ATLAS collaboration, Constraints on New Phenomena via Higgs Coupling Measurements with the ATLAS Detector, ATLAS-CONF-2014-010 (2014).

[61] ATLAS collaboration, Search for Scalar Diphoton Resonances in the Mass Range 65-600 GeV with the ATLAS Detector in pp Collision Data at $\sqrt{s}=8 \mathrm{TeV}$, Phys. Rev. Lett. 113 (2014) 171801 [arXiv: 1407.6583] [inSPIRE].

[62] CMS collaboration, Search for a Higgs Boson in the Mass Range from 145 to $1000 \mathrm{GeV}$ Decaying to a Pair of $W$ or $Z$ Bosons, JHEP 10 (2015) 144 [arXiv: 1504. 00936] [InSPIRE].

[63] ATLAS collaboration, Constraints on new phenomena via Higgs boson couplings and invisible decays with the ATLAS detector, JHEP 11 (2015) 206 [arXiv:1509.00672] [INSPIRE].

[64] ATLAS collaboration, Searches for Higgs boson pair production in the $h h \rightarrow b b \tau \tau, \gamma \gamma W W^{*}, \gamma \gamma b b, b b b b$ channels with the ATLAS detector, Phys. Rev. D 92 (2015) 092004 [arXiv: 1509.04670] [INSPIRE].

[65] ATLAS, CMS collaborations, G. Aad et al., Combined Measurement of the Higgs Boson Mass in pp Collisions at $\sqrt{s}=7$ and $8 \mathrm{TeV}$ with the ATLAS and CMS Experiments, Phys. Rev. Lett. 114 (2015) 191803 [arXiv:1503.07589] [INSPIRE].

[66] G. Bélanger et al., Automatic calculations in high energy physics and Grace at one-loop, Phys. Rept. 430 (2006) 117 [hep-ph/0308080] [INSPIRE].

[67] F. Boudjema and E. Chopin, Double Higgs production at the linear colliders and the probing of the Higgs selfcoupling, Z. Phys. C 73 (1996) 85 [hep-ph/9507396] [INSPIRE].

[68] F. Boudjema, L.D. Ninh, S. Hao and M.M. Weber, NLO corrections to $e^{+} e^{-} \rightarrow W W Z$ and $e^{+} e^{-} \rightarrow Z Z Z$, Phys. Rev. D 81 (2010) 073007 [arXiv:0912.4234] [InSPIRE].

[69] N. Baro, F. Boudjema, G. Chalons and S. Hao, Relic density at one-loop with gauge boson pair production, Phys. Rev. D 81 (2010) 015005 [arXiv:0910.3293] [INSPIRE].

[70] G. Chalons and A. Semenov, Loop-induced photon spectral lines from neutralino annihilation in the NMSSM, JHEP 12 (2011) 055 [arXiv:1110.2064] [INSPIRE].

[71] G. Chalons and F. Domingo, Analysis of the Higgs potentials for two doublets and a singlet, Phys. Rev. D 86 (2012) 115024 [arXiv:1209.6235] [INSPIRE].

[72] G. Chalons, M.J. Dolan and C. McCabe, Neutralino dark matter and the Fermi gamma-ray lines, JCAP 02 (2013) 016 [arXiv:1211.5154] [INSPIRE].

[73] G. Bélanger, V. Bizouard and G. Chalons, Boosting Higgs boson decays into gamma and a $Z$ in the NMSSM, Phys. Rev. D 89 (2014) 095023 [arXiv: 1402.3522] [INSPIRE].

[74] A.V. Semenov, LanHEP: A package for automatic generation of Feynman rules in gauge models, hep-ph/9608488 [INSPIRE]. 
[75] A. Semenov, LanHEP - a package for automatic generation of Feynman rules from the Lagrangian. Updated version 3.2, arXiv:1412.5016 [INSPIRE].

[76] B.W. Lee, C. Quigg and H.B. Thacker, Weak Interactions at Very High-Energies: The Role of the Higgs Boson Mass, Phys. Rev. D 16 (1977) 1519 [inSPIRE].

[77] M. Lüscher and P. Weisz, Is There a Strong Interaction Sector in the Standard Lattice Higgs Model?, Phys. Lett. B 212 (1988) 472 [inSPIRE].

[78] M.S. Chanowitz and M.K. Gaillard, The TeV Physics of Strongly Interacting W's and Z's, Nucl. Phys. B 261 (1985) 379 [INSPIRE].

[79] R.N. Lerner and J. McDonald, Gauge singlet scalar as inflaton and thermal relic dark matter, Phys. Rev. D 80 (2009) 123507 [arXiv:0909.0520] [INSPIRE].

[80] G. Altarelli and R. Barbieri, Vacuum polarization effects of new physics on electroweak processes, Phys. Lett. B 253 (1991) 161 [INSPIRE].

[81] M.E. Peskin and T. Takeuchi, A New constraint on a strongly interacting Higgs sector, Phys. Rev. Lett. 65 (1990) 964 [INSPIRE].

[82] M.E. Peskin and T. Takeuchi, Estimation of oblique electroweak corrections, Phys. Rev. D 46 (1992) 381 [INSPIRE].

[83] I. Maksymyk, C.P. Burgess and D. London, Beyond S, T and U, Phys. Rev. D 50 (1994) 529 [hep-ph/9306267] [INSPIRE].

[84] GFitter Group collaboration, M. Baak et al., The global electroweak fit at NNLO and prospects for the LHC and ILC, Eur. Phys. J. C 74 (2014) 3046 [arXiv:1407.3792] [INSPIRE].

[85] P. Bechtle, O. Brein, S. Heinemeyer, G. Weiglein and K.E. Williams, HiggsBounds: Confronting Arbitrary Higgs Sectors with Exclusion Bounds from LEP and the Tevatron, Comput. Phys. Commun. 181 (2010) 138 [arXiv:0811.4169] [INSPIRE].

[86] P. Bechtle, O. Brein, S. Heinemeyer, G. Weiglein and K.E. Williams, HiggsBounds 2.0.0: Confronting Neutral and Charged Higgs Sector Predictions with Exclusion Bounds from LEP and the Tevatron, Comput. Phys. Commun. 182 (2011) 2605 [arXiv:1102.1898] [InSPIRE].

[87] P. Bechtle et al., HiggsBounds-4: Improved Tests of Extended Higgs Sectors against Exclusion Bounds from LEP, the Tevatron and the LHC, Eur. Phys. J. C 74 (2014) 2693 [arXiv: 1311.0055] [INSPIRE].

[88] P. Bechtle, S. Heinemeyer, O. Stål, T. Stefaniak and G. Weiglein, HiggsSignals: Confronting arbitrary Higgs sectors with measurements at the Tevatron and the LHC, Eur. Phys. J. C 74 (2014) 2711 [arXiv: 1305.1933] [INSPIRE].

[89] ATLAS, CMS collaborations, Measurements of the Higgs boson production and decay rates and constraints on its couplings from a combined ATLAS and CMS analysis of the LHC pp collision data at $\sqrt{s}=7$ and $8 \mathrm{TeV}$, ATLAS-CONF-2015-044 (2015).

[90] T. Robens and T. Stefaniak, Singlet Benchmarks for LHC Run II, submitted to the Higgs Cross section Working Group, September 2015.

[91] T. Robens and T. Stefaniak, LHC Benchmark Scenarios for the Real Higgs Singlet Extension of the Standard Model, arXiv:1601.07880 [INSPIRE].

[92] P. Bechtle et al., Recent Developments in HiggsBounds and a Preview of HiggsSignals, PoS (CHARGED 2012) 024 [arXiv: 1301.2345] [INSPIRE].

[93] S. Heinemeyer, MSSM Higgs physics at higher orders, Int. J. Mod. Phys. A 21 (2006) 2659 [hep-ph/0407244] [INSPIRE]. 
[94] D. Lopez-Val and J. Solà, Neutral Higgs-pair production at Linear Colliders within the general 2HDM: Quantum effects and triple Higgs boson self-interactions, Phys. Rev. D 81 (2010) 033003 [arXiv:0908.2898] [INSPIRE].

[95] W. Hollik, E. Kraus, M. Roth, C. Rupp, K. Sibold and D. Stöckinger, Renormalization of the minimal supersymmetric standard model, Nucl. Phys. B 639 (2002) 3 [hep-ph/0204350] [INSPIRE].

[96] A. Sirlin, Radiative Corrections in the $\mathrm{SU}(2)_{L} \otimes \mathrm{U}(1)$ Theory: A Simple Renormalization Framework, Phys. Rev. D 22 (1980) 971 [INSPIRE].

[97] M. Böhm, H. Spiesberger and W. Hollik, On the One Loop Renormalization of the Electroweak Standard Model and Its Application to Leptonic Processes, Fortsch. Phys. 34 (1986) 687 [INSPIRE].

[98] W.F.L. Hollik, Radiative Corrections in the Standard Model and their Role for Precision Tests of the Electroweak Theory, Fortsch. Phys. 38 (1990) 165 [InSPIRE].

[99] A. Denner, Techniques for calculation of electroweak radiative corrections at the one loop level and results for W physics at LEP-200, Fortsch. Phys. 41 (1993) 307 [arXiv:0709.1075] [INSPIRE].

[100] M. Steinhauser, Leptonic contribution to the effective electromagnetic coupling constant up to three loops, Phys. Lett. B 429 (1998) 158 [hep-ph/9803313] [INSPIRE].

[101] K. Hagiwara, A.D. Martin, D. Nomura and T. Teubner, Predictions for $g-2$ of the muon and $\alpha_{\mathrm{QED}}\left(M_{Z}^{2}\right)$, Phys. Rev. D 69 (2004) 093003 [hep-ph/0312250] [INSPIRE].

[102] Particle Data Group collaboration, K.A. Olive et al., Review of Particle Physics, Chin. Phys. C 38 (2014) 090001 [INSPIRE].

[103] W. Hollik, Renormalization of the Standard Model, in Precision tests of the standard electroweak model, P. Langacker ed. (1993), pg. 37-116.

[104] W. Hollik, Twenty years of $\Delta r$ : The $W-Z$ mass correlation, J. Phys. G 29 (2003) 131 [INSPIRE].

[105] W. Hollik, Electroweak theory, J. Phys. Conf. Ser. 53 (2006) 7 [InSPIRE].

[106] D.C. Kennedy and B.W. Lynn, Electroweak Radiative Corrections with an Effective Lagrangian: Four Fermion Processes, Nucl. Phys. B 322 (1989) 1 [INSPIRE].

[107] P. Langacker, Precision tests of the standard electroweak model, World Scientific, Singapore, Advanced Series on Directions in High Energy Physics 14 (1995) 1008.

[108] M. Sperling, D. Stöckinger and A. Voigt, Renormalization of vacuum expectation values in spontaneously broken gauge theories, JHEP 07 (2013) 132 [arXiv:1305.1548] [INSPIRE].

[109] M. Gorbahn, S. Jager, U. Nierste and S. Trine, The supersymmetric Higgs sector and $B-\bar{B}$ mixing for large tan $\beta$, Phys. Rev. D 84 (2011) 034030 [arXiv:0901.2065] [INSPIRE].

[110] C. Itzykson and J.B. Zuber, Quantum Field Theory, McGraw-Hill (1980).

[111] A. Freitas and D. Stöckinger, Gauge dependence and renormalization of tan beta in the MSSM, Phys. Rev. D 66 (2002) 095014 [hep-ph/0205281] [InSPIRE].

[112] J. Guasch, J. Solà and W. Hollik, Yukawa coupling corrections to scalar quark decays, Phys. Lett. B 437 (1998) 88 [hep-ph/9802329] [INSPIRE].

[113] H. Eberl, S. Kraml and W. Majerotto, Yukawa coupling corrections to stop, sbottom and stau production in $e^{+} e^{-}$annihilation, JHEP 05 (1999) 016 [hep-ph/9903413] [INSPIRE]. 
[114] J. Guasch, W. Hollik and J. Solà, Full electroweak one loop radiative corrections to squark decays in the MSSM, Phys. Lett. B 510 (2001) 211 [hep-ph/0101086] [INSPIRE].

[115] S. Kanemura, M. Kikuchi and K. Yagyu, Radiative corrections to the Higgs boson couplings in the model with an additional real singlet scalar field, arXiv:1511.06211 [INSPIRE].

[116] P. Gambino, P.A. Grassi and F. Madricardo, Fermion mixing renormalization and gauge invariance, Phys. Lett. B 454 (1999) 98 [hep-ph/9811470] [INSPIRE].

[117] B.A. Kniehl, F. Madricardo and M. Steinhauser, Gauge independent $W$ boson partial decay widths, Phys. Rev. D 62 (2000) 073010 [hep-ph/0005060] [INSPIRE].

[118] A. Barroso, L. Brucher and R. Santos, Renormalization of the Cabibbo-Kobayashi-Maskawa matrix, Phys. Rev. D 62 (2000) 096003 [hep-ph/0004136] [InSPIRE].

[119] A. Pilaftsis, Gauge and scheme dependence of mixing matrix renormalization, Phys. Rev. D 65 (2002) 115013 [hep-ph/0203210] [INSPIRE].

[120] A. Denner, E. Kraus and M. Roth, Physical renormalization condition for the quark mixing matrix, Phys. Rev. D 70 (2004) 033002 [hep-ph/0402130] [INSPIRE].

[121] J.R. Espinosa and I. Navarro, Scale independent mixing angles, Phys. Rev. D 66 (2002) 016004 [hep-ph/0109126] [INSPIRE].

[122] J.R. Espinosa and Y. Yamada, Scale independent and gauge independent mixing angles for scalar particles, Phys. Rev. D 67 (2003) 036003 [hep-ph/0207351] [INSPIRE].

[123] G. Passarino and M.J.G. Veltman, One Loop Corrections for $e^{+} e^{-}$Annihilation Into $\mu^{+} \mu^{-}$ in the Weinberg Model, Nucl. Phys. B 160 (1979) 151 [InSPIRE].

[124] D. Binosi and J. Papavassiliou, Pinch Technique: Theory and Applications, Phys. Rept. 479 (2009) 1 [arXiv:0909.2536] [inSPIRE].

[125] J.M. Cornwall, Dynamical Mass Generation in Continuum QCD, Phys. Rev. D 26 (1982) 1453 [INSPIRE].

[126] J.M. Cornwall and J. Papavassiliou, Gauge Invariant Three Gluon Vertex in QCD, Phys. Rev. D 40 (1989) 3474 [INSPIRE].

[127] T. Hahn, Generating Feynman diagrams and amplitudes with FeynArts 3, Comput. Phys. Commun. 140 (2001) 418 [hep-ph/0012260] [INSPIRE].

[128] C. Degrande, C. Duhr, B. Fuks, D. Grellscheid, O. Mattelaer and T. Reiter, UFO - The Universal FeynRules Output, Comput. Phys. Commun. 183 (2012) 1201 [arXiv:1108.2040] [INSPIRE].

[129] A. Alloul, N.D. Christensen, C. Degrande, C. Duhr and B. Fuks, FeynRules 2.0 - A complete toolbox for tree-level phenomenology, Comput. Phys. Commun. 185 (2014) 2250 [arXiv:1310.1921] [INSPIRE].

[130] T. Hahn and M. Pérez-Victoria, Automatized one loop calculations in four-dimensions and D-dimensions, Comput. Phys. Commun. 118 (1999) 153 [hep-ph/9807565] [INSPIRE].

[131] G. Chalons, D. López-Val and T. Robens, work in progress.

[132] CMS collaboration, Constraints on the Higgs boson width from off-shell production and decay to Z-boson pairs, Phys. Lett. B 736 (2014) 64 [arXiv:1405.3455] [INSPIRE].

[133] ATLAS collaboration, Constraints on the off-shell Higgs boson signal strength in the high-mass $Z Z$ and $W W$ final states with the ATLAS detector, Eur. Phys. J. C 75 (2015) 335 [arXiv: 1503.01060] [INSPIRE]. 
[134] DELPHI, OPAL, ALEPH, LEP Working Group for Higgs Boson Searches, L3 collaborations, S. Schael et al., Search for neutral MSSM Higgs bosons at LEP, Eur. Phys. J. C 47 (2006) 547 [hep-ex/0602042] [INSPIRE].

[135] D. Buarque Franzosi, F. Maltoni and C. Zhang, Effective field theory approach to the Higgs lineshape, Phys. Rev. D 87 (2013) 053015 [arXiv:1211.4835] [INSPIRE].

[136] M. Bonvini, F. Caola, S. Forte, K. Melnikov and G. Ridolfi, Signal-background interference effects for $g g \rightarrow H \rightarrow W^{+} W^{-}$beyond leading order, Phys. Rev. D 88 (2013) 034032 [arXiv: 1304.3053] [INSPIRE].

[137] N. Kauer and C. O'Brien, Heavy Higgs signal-background interference in $g g \rightarrow V V$ in the Standard Model plus real singlet, Eur. Phys. J. C 75 (2015) 374 [arXiv:1502.04113] [INSPIRE].

[138] N. Kauer, C. O'Brien and E. Vryonidou, Interference effects for $H \rightarrow W W \rightarrow \ell \nu q \bar{q}^{\prime}$ and $H \rightarrow Z Z \rightarrow \ell \bar{\ell} q \bar{q}$ searches in gluon fusion at the LHC, JHEP 10 (2015) 074 [arXiv: 1506.01694] [INSPIRE].

[139] E. Maina, Interference effects in Heavy Higgs production via gluon fusion in the Singlet Extension of the Standard Model, JHEP 06 (2015) 004 [arXiv: 1501.02139] [INSPIRE].

[140] A. Ballestrero and E. Maina, Interference Effects in Higgs production through Vector Boson Fusion in the Standard Model and its Singlet Extension, JHEP 01 (2016) 045 [arXiv: 1506. 02257] [INSPIRE].

[141] S. Kanemura, Y. Okada, E. Senaha and C.P. Yuan, Higgs coupling constants as a probe of new physics, Phys. Rev. D 70 (2004) 115002 [hep-ph/0408364] [INSPIRE].

[142] S. Kanemura, Y. Okada and E. Senaha, Electroweak baryogenesis and quantum corrections to the triple Higgs boson coupling, Phys. Lett. B 606 (2005) 361 [hep-ph/0411354] [INSPIRE].

[143] S. Kanemura, M. Kikuchi and K. Yagyu, Fingerprinting the extended Higgs sector using one-loop corrected Higgs boson couplings and future precision measurements, Nucl. Phys. B 896 (2015) 80 [arXiv:1502.07716] [INSPIRE].

[144] M. Mühlleitner, D.T. Nhung and H. Ziesche, The order $\mathcal{O}\left(\alpha_{t} \alpha_{s}\right)$ corrections to the trilinear Higgs self-couplings in the complex NMSSM, JHEP 12 (2015) 034 [arXiv:1506.03321] [INSPIRE].

[145] A. Arhrib, R. Benbrik, J. El Falaki and A. Jueid, Radiative corrections to the Triple Higgs Coupling in the Inert Higgs Doublet Model, JHEP 12 (2015) 007 [arXiv:1507.03630] [INSPIRE]. 